\title{
Amplitude Fluctuation Effects in Shallow Water Acous- tic Scattering by Internal Waves
}

by

\author{
Lei Fu \\ B.S., Peking University, P. R. China (1990) \\ M.S., The Chinese Academy of Sciences, P. R. China (1993) \\ Submitted in partial fulfillment of the requirements for the degree of \\ MASTER OF SCIENCE IN OCEANOGRAPHIC ENGINEERING \\ at the \\ MASSACHUSETTS INSTITUTE OF TECHNOLOGY \\ and the \\ WOODS HOLE OCEANOGRAPHIC INSTITUTION
}

August 1995

\begin{tabular}{c} 
MARINE \\
BIOLOGICAL \\
LABORATORY \\
\hline LIB RARY \\
\hline $\begin{array}{c}\text { WOOOS HOLE, MASS. } \\
\text { W. H. O. I. }\end{array}$ \\
\hline
\end{tabular}

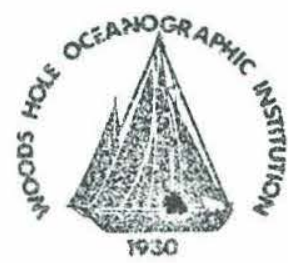

Copyright 1995, Lei Fu. All rights reserved.

The author hereby grants to MIT and WHOI permission to reproduce and to distribute copies of this thesis document in whole or in part.

Author

MIT/WHOI Joint Program in Oceanographic Engineering

Certified by................

Dr. James Lynch

Associate Scientist, Woods Hole Oceanographic Institution

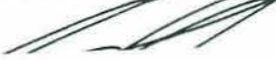

Thesis Supervisor

Certified by.
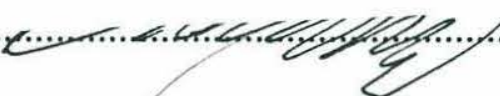

Professor Henrik Schmidt

Professor of Ocean Engineering, Massachusetts Institute of Technology

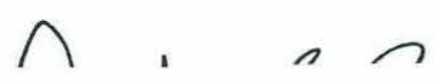

Thesis Reader

Accepted by.

Professor Arthur B. Baggereor

Chairman, MIT/WHOI Joint Committee of Oceanographic Engineering 


\title{
Amplitude Fluctuation Effects in Shallow Water Acous- tic Scattering by Internal Waves \\ by
}

\author{
Lei Fu
}

\begin{abstract}
Submitted to MIT/WHOI Joint Program in Oceanographic Engineering in partial fulfillment of the requirements for the degree of Master of Science in Oceanographic Engineering
\end{abstract}

\begin{abstract}
This thesis investigates the amplitude fluctuation effects in acoustic scattering due to shallow water internal waves. Theoretically, it uses the adiabatic approximation and perturbation methods to statistically evaluate acoustic transmission fluctuations caused by internal waves in the ocean; it also investigates acoustic mode coupling effect due to internal waves. Numerically, this thesis simulates the shallow water internal wave(IW) field using the Garrett-Munk internal wave spectrum model and then evaluates acoustic transmission in the simulated internal wave field with the Kraken normal mode program. Theoretical calculations are also performed using the theory developed in this thesis. Comparisons are made between theory and numerical calculations.

The results presented and discussed in this thesis are related to the following issues: coherent and incoherent intensity fluctuations for adiabatic approximation, acoustic mode coupling due to IW's, transmission loss difference between adiabatic and coupled mode methods, and their dependence on range, IW amplitude and frequency.
\end{abstract}

Thesis Supervisor: Dr. James Lynch

Title: Associate Scientist, Woods Hole Oceanographic Institution 


\section{Acknowledgments}

First of all, I would like to thank my thesis supervisor, Dr. James Lynch, for giving me motivation, encouragement, guidance, and help all the time during my graduate study in the MIT/WHOI joint program. He even read and edited every sentence in my thesis draft. All in all, I would like to say: thank you very much, Jim!

I would also like to thank Professor Henrik Schmidt for reading my thesis and giving me his comments from an expert point of view. I thank Professor Arthur Baggeroer, my academic advisor at MIT, for advising me on my academic study in the Joint Program.

I thank Arthur Newhall for helping me on computer work in general and on the Kraken program in particular. I also thank Peter Traykovski for giving me his IW mode codes and Michael Porter for allowing me to use two of his figures in my thesis.

Dezhang Chu and Dajun Tang offered me much help during my study at WHOI. I thank them for their help and sincerity.

Thanks also go to John Farrington, Jake Peirson, Abbie Calvin, Ronni Schwartz, and all other WHOI Education Office staff for their support of my study in the Joint Program.

My fellow students and friends at WHOI and MIT have been always a source of help, information and fun. I thank them for their help and the wonderful time we had together.

Finally, I especially thank my wife, Zhaohui and all of my family, my grandparents, my parents, my brother and my sister for their love, support, and understanding. 
I dedicate this thesis to all of my family. 


\section{Table of contents}

$\begin{array}{ll}\text { Abstract } & 2\end{array}$

$\begin{array}{ll}\text { Acknowledgments } & 3\end{array}$

$\begin{array}{ll}\text { List of Tables } & 7\end{array}$

$\begin{array}{lr}\text { List of Figures } & 8\end{array}$

1. Introduction $\quad 11$

1.1 Background 11

$\begin{array}{ll}1.2 \text { Thesis objectives and organization } & 12\end{array}$

2. Internal waves in the ocean 14

2.1 Introduction 14

2.2 Theory 14

2.3 Experimental measurement of internal waves 17

2.4 Garrett-Munk internal wave model 19

2.5 Solitons (solitary internal waves) 21

3. Acoustic scattering due to internal waves 23

3.1 Normal mode theory 23

$\begin{array}{ll}3.2 \text { Adiabatic approximation } & 27\end{array}$

3.3 Adiabatic description of acoustic scattering due to internal waves 31

3.4 Coupled mode description of acoustic scattering due to internal waves $\quad 42$

3.5 Solitons vs. linear internal waves $\quad 48$

4. Simulations and numerical calculations $\quad 51$

4.1 Realization of Garrett-Munk internal wave field 51

4.2 Acoustic waveguide model $\quad 55$

4.3 The KRAKEN normal mode program 61

4.4 Numerical results and analysis $\quad 65$ 
5. Conclusions and Future work

5.1 Conclusions

91

5.2 Recommendations for future work

93

Appendix A

Calculations of eigenvalue and eigenfunction perturbations

94

References

97 


\section{List of tables}

4.1 Horizontal wavenumbers and mode amplitudes for the background sound speed profile with frequency of $100 \mathrm{~Hz}$ and source depth of $30 \mathrm{~m}$. .58

4.2 Mode cycle distance between each pair of acoustic modes for the background sound speed profile with frequency of $100 \mathrm{~Hz}$.

4.3 Horizontal wavenumbers and mode amplitudes for the background sound speed profile with frequency of $50 \mathrm{~Hz}$ and source depth of $30 \mathrm{~m}$.

4.4 Mode cycle distance between each pair of acoustic modes for the background sound speed profile with frequency of $50 \mathrm{~Hz}$.

4.5 Horizontal wavenumbers and mode amplitudes for the background sound speed profile with frequency of $200 \mathrm{~Hz}$ and source depth of $30 \mathrm{~m}$.

4.6 Mode cycle distance between each pair of acoustic modes for the background sound speed profile with frequency of $200 \mathrm{~Hz}$. 


\section{List of figures:}

4.1 (a) Buoyancy frequency profile N(z); (b) Internal wave modes calculated from

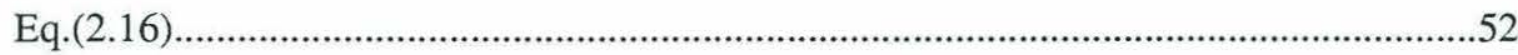

4.2 Garrett-Munk IW spectrum used for simulating the IW field..................................53

4.3 IW horizontal wavenumber spectrum for modes $1-8 \ldots \ldots \ldots \ldots \ldots \ldots \ldots \ldots \ldots \ldots \ldots \ldots \ldots \ldots \ldots \ldots . . . . .54$

4.4 One realization of IW vertical displacement from GM spectrum...........................54

4.5 One realization of IW induced sound speed fluctuations rom GM spectrum..........55

4.6 (a) Background sound speed profile; (b) mode amplitudes for source depth of 30m;

(c) acoustic modes calculated using Kraken programs. Frequency $=100 \mathrm{~Hz}$.

4.7 2-D transmission loss for the background sound speed profile model with frequency of $100 \mathrm{~Hz}$ and source depth of $30 \mathrm{~m}$.

4.8 One realization of sound speed profiles with IW induced fluctuations...................60

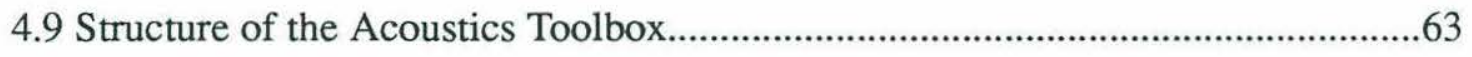

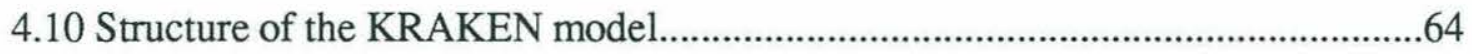

4.11 Mean and variance of acoustic intensity vs. number of realizations at range of

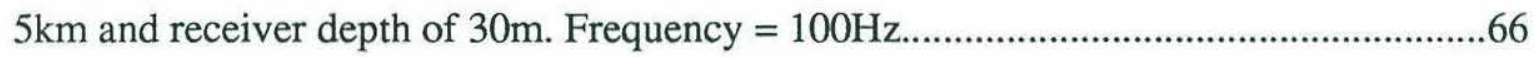

4.12 Transmission loss at receiver depth of $30 \mathrm{~m}$ for frequency of $100 \mathrm{~Hz}$ and source

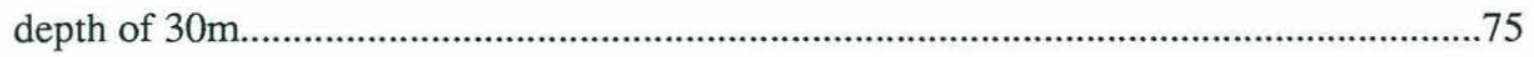

4.13 Standard deviation normalized by mean value for the acoustic intensity from adiabatic calculation. IW amplitude $=10 \mathrm{~m}$ and frequency $=100 \mathrm{~Hz}$

4.14 Standard deviation normalized by mean value for the acoustic intensity from coupled mode calculation. IW amplitude $=10 \mathrm{~m}$ and acoustic frequency $=100 \mathrm{~Hz} \ldots \ldots \ldots \ldots \ldots . . .76$

4.15 Transmission loss difference between adiabatic approximation and coupled mode calculations (average of 49 realizations). Frequency $=100 \mathrm{~Hz}$. .76 
4.16 Transmission loss difference between adiabatic approximation and coupled mode calculations. Frequency $=100 \mathrm{~Hz}$, source depth $=30 \mathrm{~m}$, and receiver depth $=10 \mathrm{~m}$ .77

4.17 Acoustic horizontal wavenumber perturbations vs. range for one realization of IW induced sound speed fluctuations. Frequency $=100 \mathrm{~Hz}$ .78

4.18 Modal phase perturbations vs. range due to the horizontal wavenumber perturbations shown in Fig. 4.17 79

4.19 TL difference between adiabatic approximation and coupled mode calculations (average of 49 realizations) for frequency of $100 \mathrm{~Hz}$ and IW amplitude of $20 \mathrm{~m}$. .80

4.20 TL difference between adiabatic approximation and coupled mode calculations (average of 49 realizations) for frequency of $100 \mathrm{~Hz}$ and IW amplitude of $30 \mathrm{~m}$. .80

4.21 TL difference between adiabatic and coupled mode calculations (average of 49 realizations) for IW amplitude of $10 \mathrm{~m}, 20 \mathrm{~m}, 30 \mathrm{~m}$ at receiver depth of $10 \mathrm{~m}$ and $30 \mathrm{~m} \ldots \ldots . .81$

4.22 The average ratio of standard deviation to mean value of coherent intensity for different amplitudes. Frequency $=100 \mathrm{~Hz}$ 82

4.23 TL difference between adiabatic and coupled mode calculations (average of 49 realizations) for frequency of $50 \mathrm{~Hz}$.

4.24 TL difference between adiabatic and coupled mode calculations (average of 49 realizations) for frequency of $200 \mathrm{~Hz}$.

4.25 TL difference between adiabatic and coupled mode calculations (average of 49 realizations) for different frequencies at receiver depth of $10 \mathrm{~m}$ and $30 \mathrm{~m}$. 84

4.26 The average ratio of standard deviation to mean value of coherent intensity for different frequencies at different receiver depths. IW amplitude and source depth are the same. .85

4.27 Coherent and incoherent transmission loss curves at receiver depth of $30 \mathrm{~m}$ for IW amplitude of $20 \mathrm{~m}$, frequency of $100 \mathrm{~Hz}$ and source depth of $30 \mathrm{~m}$ 88

4.28 Coherent and incoherent transmission loss curves at receiver depth of $30 \mathrm{~m}$ for IW amplitude of $30 \mathrm{~m}$, frequency of $100 \mathrm{~Hz}$, and source depth of $30 \mathrm{~m}$ .88 
4.29 The ratio (average of 49 realizations) of standard deviation to mean value of incoherent intensity (Kraken results). Frequency $=100 \mathrm{~Hz}$, IW amplitude $=20 \mathrm{~m}$, and source

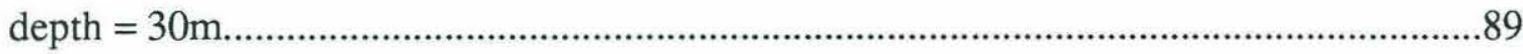

4.30 The ratio (average of 49 realizations) of standard deviation to mean value for incoherent intensity (theoretical results). Frequency $=100 \mathrm{~Hz}$, IW amplitude $=20 \mathrm{~m}$, and source depth $=30 \mathrm{~m}$ .89

4.31 The average ratio of standard deviation to mean value of incoherent intensity (Kraken results) vs. IW amplitude at different depths

.90 


\section{Chapter 1}

\section{Introduction}

\subsection{Background}

Sound waves, with much lower attenuation than electromagnetic waves in water, can propagate very long distances in the ocean and thus have been utilized in many military and civilian applications, such as sonar systems, underwater communication, ocean exploration, etc. Spurred on by the usefulness of underwater sound, researchers have been making progress in every area of ocean acoustics. An acoustical signal received at a point in the ocean from a remote source can vary considerably in amplitude, travel time, and even the direction from which it arrives because of inhomogeneities and fluctuations in the ocean environment. These effects might seriously degrade the performance of sonar systems and many other underwater applications. However, one man's noise could be another's signal. The acoustic fluctuations also may provide us a valuable way to explore the ocean. Thus research into acoustic fluctuations due to various oceanic processes has been drawing much effort[1][2]. The process that this thesis will concentrate on is internal waves in the ocean.

Internal waves(IW's) are similar to ordinary sea surface waves except that they occur within the sea rather than at the surface. They exist at interfaces between water layers of different density, especially at the pycnocline. In a homogeneous sea, they can not exist. Internal waves characteristically have greater amplitudes and slower speeds of propagation than do surface waves. Internal wave induced sound speed fluctuations cause acoustic scattering when sound waves propagate through a water column with internal wave activity. 
Much of the work on acoustic scattering by internal waves has been concerned with the deep ocean. In a book edited by Flatte [1], a clear overview is given of the work in this area up until 1979. There was also theoretical work on the statistics of normal mode amplitudes in the internal wave field by Dozier and Tappert [3][4]. As for the work on acoustic scattering by shallow water internal waves, some highlights are the study of resonant acoustic scattering by internal wave solitons by Zhou, Zhang, and Rogers[5], the study of acoustic modal wavenumber fluctuations by Essen[6], and the studies of internal wave induced phase front curvature across horizontal arrays by Shmelerv[7], Ruben-

stein[8], and their co-workers. The first Zhou et al study concentrated on acoustic amplitude attenuation effect, and the latter two studies focused on phase fluctuations. In recent work by Lynch et al.[9] and Traykovski[10], acoustic travel time perturbations due to shallow water internal waves and internal tides in the Barents Sea Polar Front have been studied.

\subsection{Thesis objectives and organization}

In this thesis, we will investigate the amplitude fluctuation effects in acoustic scattering due to linear shallow water internal waves. We mainly use adiabatic approximation method to describe "weak scattering" due to linear internal waves. We will also look at mode coupling effect and compare acoustic scattering by linear internal waves with that by solitons. The main argument we would like to make is that for linear internal waves, when the mode cycle distances are much less than the "dominant" IW wavelength, the coupling is small and the adiabatic approximation is valid. 
This thesis is organized as follows. Chapter 1 is the introduction. Chapter 2 is about internal waves in the ocean: theory, observation techniques, data analysis, the GarrettMunk(GM) model, and solitons. Chapter 3 is the main theoretical part of this thesis in which we develop the full theory for coherent and incoherent intensity fluctuations due to internal waves and describe the resonant coupling effect. Chapter 4 contains simulations and numerical calculations; specifically we make simulations of the Garrett-Munk IW model, use the Kraken normal mode program to evaluate the acoustic field in the simulated internal wave environment, do calculations using the theories developed in Chapter 3, and then discuss the results. We present conclusions in Chapter 5.

Chapter 1 and 2 are generally a review of previous work. Chapters 3-5 are mainly my own contributions. 


\section{Chapter 2}

\section{Internal waves in the ocean}

\subsection{Introduction}

Internal waves occur beneath the sea surface between water layers of different density. These gravity waves propagate along a pycnocline associated with either a halocline or thermocline. The causes of internal waves are varied and not completely understood. Some causes are: flow over bathymetry, storms, surface waves, tidal action, wind blowing over the sea surface, etc. Internal waves travel more slowly than surface waves, but can attain much greater amplitudes. They mix water below the surface and may be important in the movement of sediments.

Internal waves can be found in both shallow and deep ocean water and in large freshwater lakes, such as Lake Ontario. "Typical” characteristics of internal waves are as follows[11]: in shallow water, the internal waves have periods of 4 minutes to 25 hours, amplitudes of up to $20 \mathrm{~m}$, and speeds of order of $5 \mathrm{~cm} / \mathrm{sec}$; in deep ocean, the internal waves have periods of 4 minutes to 25 hours, amplitudes of up to $100 \mathrm{~m}$, and speeds of $100 \mathrm{~cm} / \mathrm{sec}$.

\subsection{Theory}

Using the basic equations of momentum and continuity for a fluid, an exact modal solution for internal waves can be derived. The momentum equation for an isotropic fluid in the absence of viscous effect is given by [12]

$$
\frac{D \vec{u}}{D t}+2 \vec{\Omega} \times \vec{u}=-\rho^{-1} \nabla p-\vec{g}
$$


and the continuity equation for an incompressible fluid is given by

$$
\nabla \cdot \vec{u}=\frac{\partial u}{\partial x}+\frac{\partial v}{\partial y}+\frac{\partial w}{\partial z}=0
$$

Applying small perturbation terms $p^{\prime}$ and $\rho^{\prime}$, the perturbed pressure and density are then given respectively by

$$
\begin{gathered}
p=-g \rho z+p^{\prime} \\
\rho=\rho_{0}+\rho^{\prime}
\end{gathered}
$$

Substituting Eqs. (2.3) and (2.4) into Eq. (2.1), the momentum equation is then linearized by neglecting the high order perturbation terms, i.e. products of perturbation quantities, and becomes

$$
\begin{gathered}
\frac{\partial u}{\partial t}-f v=-\frac{1}{\rho_{0}} \frac{\partial p^{\prime}}{\partial x} \\
\frac{\partial v}{\partial t}-f u=-\frac{1}{\rho_{0}} \frac{\partial p^{\prime}}{\partial y} \\
\frac{\partial w}{\partial t}=-\frac{1}{\rho_{0}} \frac{\partial p^{\prime}}{\partial z}-\frac{\rho^{\prime}}{\rho_{0}} g
\end{gathered}
$$

where $\mathrm{f}$ is the inertial or "local Coriolis" frequency. We now assume a separable solution for the vertical velocity $w(x)$ of the form

$$
w(\grave{x}, t)=\hat{w}(z) \exp [i(k x+l y-\omega t)]
$$

The vertical velocity $w(\vec{x})$ satisfies the boundary conditions of no normal flow at the bottom, i.e.

$$
w(\vec{x})=0 \text { at } z=H \text { (bottom) }
$$


and that a particle on the free surface remains on it

$$
w(\vec{x})=\frac{\partial \eta}{\partial t} \text { at } z=\eta(\text { surface) }
$$

which can be applied at $z=0$ since $\eta$ is small. This approximation introduces little error since the vertical displacement at the air-water interface is smaller than the maximum amplitude by about 1000 . By manipulating equations (2.3-2.8), we can obtain

$$
\frac{\partial^{2} \hat{w}}{\partial z^{2}}+k_{h}^{2}\left[\frac{N^{2}(z)-\omega^{2}}{\omega^{2}-f^{2}}\right] \hat{w}=0
$$

where $k_{h}$ is the horizontal wavenumber that satisfies

$$
k_{h}^{2}=k^{2}+l^{2}
$$

and where $N(z)$ is the buoyancy frequency which is defined as

$$
N^{2}=-\frac{g}{\rho_{0}} \frac{d \rho}{d z}
$$

The propagating wave solution for Eq. (2.11) only exits when $f<\omega<N$. Using the relationship

$$
\hat{w}=\frac{\partial \xi}{\partial t}
$$

where $\xi$ is the vertical particle displacement, and substituting it into Eq.(2.11) gives

$$
\frac{\partial^{2} \xi}{\partial z^{2}}+k_{h}\left[\frac{N^{2}(z)-\omega^{2}}{\omega^{2}-f^{2}}\right] \xi=0
$$


with the following boundary conditions:

$$
\xi(z)=0 \text { at } z=0 \text { (surface) }
$$

and

$$
\xi(z)=0 \text { at } z=H \text { (bottom). }
$$

This equation can be formulated as an eigenvalue equation and is easily solved using numerical methods such as finite element methods. The exact modal solution is

$$
\xi=\sum_{i=1}^{\infty} A_{l} \phi_{l}(z) \exp \left[i\left(k_{x l} x+k_{y l} y-\omega t\right)\right]
$$

where $l=1,2, \ldots$ is the mode number, $A_{l}$ is the amplitude, and $\phi_{l}(z)$ is the internal wave mode eigenfunction. In cylindrical coordinates, Eq.(2.18) can be expressed as

$$
\xi(r, z)=\sum_{i=1}^{\infty} A_{l}\left(k_{r}, \omega\right) \phi_{l}(z) \exp \left[i\left(k_{r} r-\omega t\right)\right]
$$

where $k_{r}$ is the horizontal wavenumber.

\subsection{Experimental measurement of internal waves}

A variety of observational techniques are now available to measure internal waves in the ocean. Among them are moored sensors, towed sensors, dropped instruments[13], and remote sensing[14][15]. Also, because internal wave-induced variations in sound speed has strong effects on acoustical signal fluctuations, acoustic transmission measurement can provide a measure of certain statistical properties of the internal wave field[6][16]. 
Current meters, temperature sensors, and vertical temperature gradient sensors, which are attached to a more or less vertical mooring line between an anchor on the seafloor and a buoyant float at or below the sea surface, provide time series of current speed and direction, temperature fluctuations, and vertical temperature gradient which give us temporal measurements of the ocean process. Usually a number of sensors are used at different vertical spacings on the same mooring, or on several moorings separated by various distances horizontally. The relationships between simultaneous measurements at different places provide the spatial information on the ocean processes.

A thermistor chain, consisting of a cable with sensors every a few meters, can be suspended below a ship and towed slowly through the upper layer of the ocean, mapping out a two-dimensional section of temperature structure down to about $200 \mathrm{~m}$ below the surface.

Instruments lowered from a hove-to vessel, or dropped freely, are very traditional ways to measure the vertical structure of the oceans. The XBT (expendable bathythermograph) can be dropped from a ship to measure the temperature profile. The CTD (conductivity, temperature, depth) records electrical conductivity and temperature (and hence salinity and density) as functions of depth as it is lowered from a stationary ship.

Remote sensing images from aircraft and satellites provide another way to observe the internal waves in the ocean. A nice example is the signatures of internal waves which were detected repeatedly in the Gulf of California by the Seasat synthetic aperture radar(SAR) [15].

Given a series of data points obtained from various instruments, which bear the temporal and spatial information, the problem then is how to interpret them, i.e. how to relate them to internal waves. A basic tool in the interpretation of a series of data points is the 
power spectrum. A time series can be related to a frequency spectrum and a spatial data series can be related to a wavenumber spectrum.

\subsection{Garrett-Munk internal wave model}

Based on experimental data on power spectra and cross spectra from many different sources, together with some simplifying assumptions, Garrett and Munk came up with a simple model describing the deep ocean distribution of internal wave energy in wavenumber-frequency space. The description of the model is as follows [17].

All quantities are nondimensionalized with reference to the deep ocean buoyancy scale depth $\mathrm{b}(1.3 \mathrm{~km})$ and the buoyancy frequency $n_{0}(3 \mathrm{cph})$ at the top of the thermocline. Horizontal wavenumber $\alpha\left(\alpha_{1}, \alpha_{2}\right)$ and vertical wavenumber $\beta$ are related to the mode number $\mathrm{j}(1,2, \ldots)$ and frequency $\omega$ according to the approximate formulas

$$
\begin{gathered}
\alpha=j \pi\left(\omega^{2}-f^{2}\right)^{1 / 2} \\
\beta=j \pi N(z)
\end{gathered}
$$

where $f$ is the inertial frequency and $N(z)$ is the buoyancy frequency. If horizontal isotropy is assumed, various forms of the energy spectrum are related according to

$$
\begin{aligned}
& \iiint E\left(\alpha_{1}, \alpha_{2}, \beta\right) d \alpha_{1} d \alpha_{2} d \beta=\iint E(\alpha, \beta) d \alpha d \beta \\
&=\iint E(\alpha, \omega) d \alpha d \omega=\iint E(\beta, \omega) d \beta d \omega=E
\end{aligned}
$$

where $\mathrm{E}$ is a dimensionless constant related to the IW energy per unit area. With this and the dispersion relations, we can make transformations into various frequency-wavenumber spaces. The mode number scale $j_{*}$ and associated wavenumbers are 


$$
\alpha_{*}=j_{*} \pi\left(\omega^{2}-f^{2}\right)^{1 / 2}, \beta_{*}=j_{*} \pi N(z), j_{*}=6
$$

Most of the energy is contained in wavenumbers less than $\alpha_{*}$ and $\beta_{*}$ according to

$$
A(\lambda)=(t-1)(1+\lambda)^{-t}, \lambda=\alpha / \alpha_{*}=\beta / \beta_{*}, t=2.5
$$

Further, set

$$
B(\omega)=2 \pi^{-1} f \omega^{-2} \gamma^{-1}, \gamma=\left(1-f^{2} / \omega^{2}\right)^{1 / 2}
$$

for $f<\omega<N(z)$, and zero otherwise. The functions A and B are so normalized that

$$
\int_{0}^{\infty} A(\lambda) d \lambda=1, \int_{0}^{\infty} B(\omega) d \omega=1
$$

With all the above definitions and assumptions, the energy spectrum is specified as

$$
E(\alpha, \omega)=E A\left(\alpha / \alpha_{*}\right) B(\omega) / \alpha_{*}, E=6.3 \times 10^{-5}
$$

This includes the similarity assumption that the shape of the spectrum as a function of horizontal wavenumber is invariant but for a scale factor, $\alpha_{*}$. Following the rules for transformation, we obtain

$$
\begin{gathered}
E(\beta, \omega)=E A\left(\beta / \beta_{*}\right) B(\omega) / \beta_{*} \\
E(\alpha, \beta)=\frac{2 \pi^{-1} f E N(z)\left(\beta / \beta_{*}\right) A\left(\beta / \beta_{*}\right)}{N^{2}(z) \alpha^{2}+f^{2} \beta^{2}} \\
0 \leq \alpha \leq \beta\left[1-f^{2} / N^{2}(z)\right]^{1 / 2}
\end{gathered}
$$

The frequency spectrum is simply

$$
E(\omega)=\int E(\alpha, \omega) d \alpha
$$




\subsection{Solitons(solitary internal waves)}

Solitons (solitary internal waves) are discrete non-dispersive packets of internal waves which are of much shorter wavelength and larger velocity than the usual linear internal waves. Solitons have been observed in many coastal zones of the world such as[5]: Massachusetts Bay, the New York Bight, the Gulf of California, the Andaman Sea offshore Thailand, the Australian North West Shelf, the Sulu Sea between the Philippines and Borneo, off the coast of Portugal, off the Hainan Island in the South China Sea, off the Strait of Gibraltar in the Alboran Sea, the Scotian Shelf off Nova Acotis, the Celtic Sea, and so on. The experimental data on them includes: current and temperature measurements, vertical profiles from CTD, XBT, and acoustic echo sounding devices, and radar and satellite images.

The generation mechanism of solitary internal waves (SIW) has long been a research area in the geophysics and fluid mechanics communities[18]. In shelf regions, stratification often has a pronounced two-layer character and a thermocline (i.e the interface between water layers with different temperatures) is established. The most non-linear hydrodynamic process occurring in a shelf thermocline is a bore, which is a stepwise variation of the thermocline level which is often accompanied by large-amplitude oscillations. Bores usually generates intense pulselike short-period waves which may be associated with soliton formation. The Korteweg-deVries (K-dV) equation models the transformation of a bore as the decomposition of a stepwise perturbation into a sequence of solitons. In the coastal seas connected to the open ocean, some experiments show that solitons are caused by the transformation of barotropic semidiurnal tides into baroclinic motions. Internal tide solitons occur when tidal excursions are greater than or equal to the topo- 
graphic length scale or when the Froude number is greater than or equal to 1(tidal current speed >internal wave speed).

Recent experiments suggest that SIWs are rather typical elements of internal motions, not only in the shelf zone but also in deep waters, at least up to hundreds of kilometers from the shelf. The observations of SIW in the deep ocean have been reported by many researchers[18]. The available data show that the SIW may appear both on the seasonal and on the main, permanent pycnoclines.

The main cause of the generation of SIWs in the ocean is the semidiurnal tide. And this phenomenon is a typical final result of the transformation of barotropic tides into baroclinic motions. The adequate theoretical description of this problem is still open in many cases because of the lack of hydrographic data. However, we can expect significant advances in this area because of more and more advanced experimental schemes. 


\section{Chapter 3}

\section{Acoustic scattering due to internal waves}

\subsection{Normal mode theory}

The formal derivation of ocean acoustics normal mode theory can be found in many books about propagation.[19] Here we will briefly go through the derivation of normal mode theory for the case of point source in a cylindrical geometry to provide the foundation for later theoretical work. The derivations in Sec. 3.1 and Sec. 3.2 mainly follow the book by Jensen et al. [20].

We begin with the Helmholtz equation in two dimensional space with sound speed and density depending only on depth z:

$$
\frac{1}{r} \frac{\partial}{\partial r}\left(r \frac{\partial p}{\partial r}\right)+\rho(z) \frac{\partial}{\partial z}\left(\frac{1}{\rho(z)} \frac{\partial p}{\partial z}\right)+\frac{\omega^{2}}{c^{2}(z)} p=-\frac{\delta(r) \delta\left(z-z_{s}\right)}{2 \pi r} .
$$

Using the technique of separation of variables, we seek a solution of the unforced equation in the form of $p(r, z)=\Phi(r) \Psi(z)$. After substituting into the above equation and dividing through by $\Phi(r) \Psi(z)$, we find

$$
\frac{1}{\Phi}\left[\frac{1}{r} \frac{d}{d r}\left(r \frac{d \Phi}{d r}\right)\right]+\frac{1}{\Psi}\left[\rho(z) \frac{d}{d z}\left(\frac{1}{\rho(z)} \frac{d \Psi}{d z}\right)+\frac{\omega^{2}}{c^{2}(z)} \Psi\right]=0
$$

The contents in the square brackets are functions of $\mathrm{r}$ and $\mathrm{z}$ respectively. Thus, the only way the equation can be satisfied is if each component is equal to a constant. Denoting this separation constant by $k_{r m}^{2}$, we obtain the modal equation, 
$\rho(z) \frac{d}{d z}\left[\frac{1}{\rho(z)} \frac{d}{d z} \Psi_{m}(z)\right]+\left[\frac{\omega^{2}}{c^{2}(z)}\right] \Psi_{m}(z)=k_{r m}^{2} \Psi_{m}(z)$

with $\Psi(0)=0,\left.\frac{d \Psi}{d z}\right|_{z=D}=0$.

Here, $\Psi_{m}(z)$ denotes the particular function $\Psi(z)$ obtained with the separation constant $k_{r m}$. The boundary conditions imposed imply a pressure-release surface located at $\mathrm{z}=0$ and a perfectly rigid bottom located at $\mathrm{z}=\mathrm{D}$. The modal equation is a classical Sturm-Liouville eigenvalue problem whose properties are well-known. (We assume for the moment that $\rho(z)$ and $c(z)$ are real functions). The modes of such Sturm-Liouville problems are orthogonal, i.e.

$$
\int_{0}^{D} \frac{\Psi_{m}(z) \Psi_{n}(z)}{\rho(z)} d z=0 \text { for } m \neq n
$$

The solutions of the modal equation are arbitrary to a multiplicative constant as is easily seen from Eq.(3.3). In order to simplify the results, we shall assume that the modes are normalized so that

$$
\int_{0}^{D} \frac{\Psi_{m}^{2}(z)}{\rho(z)} d z=1
$$

Finally, the modes form a complete set, which means we can represent an arbitrary function as a sum of the normal modes. Thus we write the pressure as

$$
p(r, z)=\sum_{m=1}^{\infty} \Phi_{m}(r) \Psi_{m}(z)
$$

Substituting this into Eq.(3.1), we obtain 


$$
\begin{gathered}
\sum_{m=1}^{\infty}\left\{\frac{1}{r} \frac{d}{d r}\left(r \frac{d}{d r} \Phi_{m}(\mathrm{r})\right) \Psi_{m}(\mathrm{z})+\Phi_{m}(\mathrm{r})\left[\rho(z) \frac{d}{d z}\left(\frac{1}{\rho(z)} \frac{d}{d z} \Psi_{m}(\mathrm{z})\right)+\frac{\omega^{2}}{c^{2}(z)} \Psi_{m}(z)\right]\right\} \\
=-\frac{\delta(r) \delta\left(z-z_{s}\right)}{2 \pi r}
\end{gathered}
$$

The term in square brackets can be further simplified using the modal equation(3.3). This yields

$$
\sum_{m=1}^{\infty}\left\{\frac{1}{r} \frac{d}{d r}\left(r \frac{d}{d r} \Phi_{m}(\mathrm{r})\right) \Psi_{m}(\mathrm{z})+k_{r m}^{2} \Phi_{m}(\mathrm{r}) \Psi_{m}(z)\right\}=-\frac{\delta(r) \delta\left(z-z_{s}\right)}{2 \pi r}
$$

Next we apply the operation

$$
\int_{0}^{D}(*) \frac{1}{\rho(z)} \Psi_{n}(z) d z
$$

to Eq.(3.7). Because of the orthogonality property given in Eq.(3.4), only the nth term in the sum remains, yielding

$$
\frac{1}{r} \frac{d}{d r}\left[r \frac{d}{d r} \Phi_{n}(r)\right]+k_{r n}^{2} \Phi_{n}(r)=-\frac{\delta(r) \Psi_{n}\left(z_{s}\right)}{2 \pi r \rho\left(z_{s}\right)}
$$

This is a standard equation whose solution is given in terms of the Hankel functions as

$$
\Phi_{n}(r)=\frac{i}{4 \rho\left(z_{s}\right)} \Psi_{n}\left(z_{s}\right) H_{0}^{(1,2)}\left(k_{r n} r\right)
$$

The choice of $H_{0}^{(1)}$ or $H_{0}^{(2)}$ is determined by the radiation condition stating that energy should be radiating outward as $r \rightarrow \infty$. Since we have suppressed a time dependence of the form $\exp (-i \omega t)$, we shall take the Hankel function of the first kind. Putting this all together, we find that 


$$
p(r, z)=\frac{i}{4 \rho\left(z_{s}\right)} \sum_{m=1}^{\infty} \Psi_{m}\left(z_{s}\right) \Psi_{m}(z) H_{0}^{(1)}\left(k_{r m} r\right)
$$

or, using the asymptotic approximation to the Hankel function,

$$
p(r, z) \approx \frac{i}{\rho\left(z_{s}\right) \sqrt{8 \pi r}} e^{-i \pi / 4} \sum_{m=1}^{\infty} \Psi_{m}\left(z_{s}\right) \Psi_{m}(z) \frac{e^{i k_{r m} r}}{\sqrt{k_{r m}}} .
$$

This is the expression for complex pressure field. Transmission loss is defined by

$$
T L(r, z)=-20 \log \left|\frac{p(r, z)}{p_{0}(r=1)}\right|
$$

where

$$
p_{0}(r)=\frac{e^{i k_{0} r}}{4 \pi r}
$$

is the pressure for the source in free space. Thus one may write

$$
T L(r, z) \cong-20 \log \left|\frac{1}{\rho\left(z_{s}\right)} \sqrt{\frac{2 \pi}{r}} \sum_{m=1}^{\infty} \Psi_{m}\left(z_{s}\right) \Psi_{m}(z) \frac{e^{i k_{r m} r}}{\sqrt{k_{r m}}}\right| .
$$

In some cases it is useful to calculate an incoherent transmission loss defined by

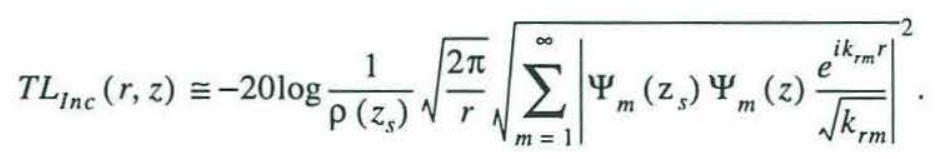

When comparing theory to measured data which has been averaged over frequency, one can often simulate the resulting smoothed transmission loss by an incoherent modal summation. Incoherent transmission loss is often appropriate for shallow-water problems, 
where the modes are bottom-interacting. Since bottom properties are often poorly known, the detailed interference pattern predicted by a coherent transmission loss calculation is sometimes not useful.

\subsection{Adiabatic approximation}

In the last section, we derived the normal mode equations for the range-independent problem. But the real ocean environment is always range-dependent. The goal of this thesis is to begin to explore the range-dependent problem introduced by internal waves. As we will see in the following, the range-dependence causes coupling, i.e. energy transferring between different modes. Numerically, one way to deal with range-dependent problems is to divide the range axis into a number of segments and approximate the field as range independent within each segment. The solution within a range-independent segment is constructed using the standard normal-mode solution and then the interface conditions (continuity of pressure and normal velocity) are used to "glue" the solutions together. This is called the step-wise coupled mode method. The coupled mode method is straightforward but leads to a somewhat computationally intensive procedure. Thus the "adiabatic approximation" method was introduced, which simplifies the above coupled-mode problem by ignoring (1) the backscattered component of the field and (2) coupling between different-order modes at the segment interfaces. This approximation was originally introduced by Pierce[21] based on analogous results for the Schrodinger equation. The derivation here follows sections of the textbook by Jensen et al[20]. To derive this approximation, we return to the Helmholtz equation in cylindrical coordinates, 


$$
\frac{1}{\rho} \frac{\partial}{\partial r}\left(r \frac{\partial p}{\partial r}\right)+\rho(z) \frac{\partial}{\partial z}\left(\frac{1}{\rho(z)} \frac{\partial p}{\partial z}\right)+\frac{\omega^{2}}{c^{2}(r, z)} p=-\frac{\delta(r) \delta\left(z-z_{s}\right)}{2 \pi r} .
$$

Since the modes form a complete set, we can represent the solution at any range as a sum of "local modes". We therefore seek a solution of the range-dependent problem in the form

$$
p(r, z)=\sum_{m} \Phi_{m}(r) \Psi_{m}(r, z)
$$

where $\Psi_{m}(r, z)$ are the local modes defined by

$$
\rho(r, z) \frac{\partial}{\partial z}\left[\frac{1}{\rho(r, z)} \frac{\partial}{\partial z} \Psi_{m}(r, z)\right]+\left[\frac{\omega^{2}}{c^{2}(r, z)}-k_{r m}^{2}(r)\right] \Psi_{m}(r, z)=0 .
$$

Thus, at any range $r, \Psi_{m}(r, z)$ is found by solving the depth-separated modal equation with the environmental properties at that range. Substituting into the Helmholtz equation yields

$$
\sum_{m} \frac{\rho}{r} \frac{\partial}{\partial r}\left(\frac{r}{\rho} \frac{\partial}{\partial r}\left(\Phi_{m} \Psi_{m}\right)\right)+\sum_{m} k_{r m}^{2}(r) \Phi_{m} \Psi_{m}=\frac{\delta(r) \delta\left(z-z_{s}\right)}{2 \pi r}
$$

where we have used Eq.(3.20) to eliminate the $z$-derivatives. Rearranging terms leads to

$$
\sum_{m}\left[\frac{\rho}{r} \frac{\partial}{\partial r}\left(\frac{r}{\rho}_{\partial r}^{\partial \Phi_{m}}\right) \Psi_{m}+2 \frac{\partial \Phi_{m} \partial \Psi_{m}}{\partial r} \bar{m}^{\rho}+\frac{\rho}{r} \frac{\partial}{\partial r}\left(\frac{r}{\rho}_{\partial r}^{\partial \Psi^{m}}\right) \Phi_{m}\right]+\sum_{m} k_{r m}^{2}(r) \Phi_{m} \Psi_{m}=-\frac{\delta(r) \delta\left(z-z_{s}\right)}{2 \pi r}
$$

For simplicity, we now assume that $\rho$ is independent of $r$. Then we apply the operator

$$
\int(*) \frac{\Psi_{n}(r, z)}{\rho} d z
$$

and because of the orthogonality property, many of the terms in the sum will disappear. 
The result is

$$
\frac{1}{r} \frac{d}{d r}\left(r \bar{d}_{n}^{n}\right)+\sum_{m} 2 B_{m n} \frac{d \Phi_{m}}{d r}+\sum_{m} A_{m n} \Phi_{m}+k_{r n}^{2}(r) \Phi_{n}=\frac{\delta(r) \Psi_{n}\left(z_{s}\right)}{2 \pi r}
$$

where

$$
\begin{gathered}
A_{m n}=\int \frac{1}{r} \frac{\partial}{\partial r}\left(r_{\frac{\partial}{\partial r}}{ }^{m}\right) \frac{\Psi_{n}}{\rho} d z, \\
B_{m n}=\int \frac{\partial \Psi_{m}}{\partial r} \frac{\Psi_{n}}{\rho} d z .
\end{gathered}
$$

Note that $B_{m n}=-B_{n m}$, since differentiating

$$
\int \frac{\Psi_{m}(z) \Psi_{n}(z)}{\rho(z)} d z=\delta_{m n}
$$

gives

$$
\left(\int \frac{\partial \Psi_{m}}{\partial r} \frac{\Psi_{n}}{\rho} d z\right)+\left(\int \frac{\partial \Psi_{n} \Psi_{m}}{\partial r} \frac{\Psi_{m}}{\rho} d z\right)=0
$$

Equations (3.24) is a statement of coupled modes written for the case of continuous variation of sound speed. It can be solved directly by, for instance, finite-differences. The adiabatic approximation can now be stated simply as the assumption that the coupling matrices $A_{m n}$ and $B_{m n}$ are negligible. We then obtain a set of decoupled equations,

$$
\frac{1}{r} \frac{d}{d r}\left(r \bar{\Phi}_{n}^{n}\right)+k_{r n}^{2}(r) \Phi_{n}=-\frac{\delta(r) \Psi_{n}\left(z_{s}\right)}{2 \pi r}
$$

which in the WKB approximation has the solution

$$
\Phi_{n}(r)=\frac{A e^{i \int_{0}^{r} k_{r n}\left(r^{\prime}\right) d r^{\prime}}}{\sqrt{k_{r n}(r)}}
$$


The value of $A$ is found by requiring that the WKB solutions match our standard solution, Eq.(3.13), when the problem is range-independent. Thus

$$
A=\frac{i}{\rho\left(z_{s}\right) \sqrt{8 \pi r}} e^{-i \pi / 4} \Psi_{n}\left(z_{s}\right)
$$

By substituting this back to Eq.(3.13), we get the final result,

$$
p(r, z)=\frac{i}{\rho\left(z_{s}\right) \sqrt{8 \pi r}} e^{-i \pi / 4} \sum_{m=1}^{\infty} \Psi_{m}\left(z_{s}\right) \Psi_{m}(r, z) \frac{e^{i \int_{0}^{r} k_{r m}\left(r^{\prime}\right) d r^{\prime}}}{\sqrt{k_{r m}(r)}}
$$

The problem with this expression is that it fails to satisfy reciprocity. So instead of using this expression, we use the following modified adiabatic formula:

$$
p(r, z)=\frac{i}{\rho\left(z_{s}\right) \sqrt{8 \pi}} e^{-i \pi / 4} \sum_{m=1}^{\infty} \Psi_{m}\left(z_{s}\right) \Psi_{m}(r, z) \frac{e^{i \int_{0}^{r} k_{r m}\left(r^{\prime}\right) d r^{\prime}}}{\sqrt{\int_{0}^{r} k_{r m}\left(r^{\prime}\right) d r^{\prime}}}
$$

This formula may be formally derived by assuming that the environment is invariant with respect to translations perpendicular to the radial connecting the source to the receiver. Having the above equation for pressure, we can now write the transmission loss expression for adiabatic approximation:

$$
T L(r, z)=-20 \log \left|\frac{\sqrt{2 \pi}}{\rho\left(z_{s}\right)} \sum_{m=1}^{\infty} \Psi_{m}\left(z_{s}\right) \Psi_{m}(r, z) \frac{e^{i \int_{0}^{r} k_{r m}\left(r^{\prime}\right) d r^{\prime}}}{\sqrt{\int_{0}^{r} k_{r m}\left(r^{\prime}\right) d r^{\prime}}}\right|
$$

and the incoherent transmission loss is: 


$$
T L_{I n c}(r, z)=-20 \log \left|\frac{\sqrt{2 \pi}}{\rho\left(z_{s}\right)} \sqrt{\sum_{m=1}^{\infty}\left|\Psi_{m}\left(z_{s}\right) \Psi_{m}(r, z) \frac{1}{\sqrt{\int_{0}^{r} k_{r m}\left(r^{\prime}\right) d r^{\prime}}}\right|}\right|^{2}
$$

\subsection{Adiabatic description of acoustic scattering due to internal waves}

Now we will use adiabatic approximation and perturbation methods to evaluate acoustic scattering due to IW's. Whether the adiabatic approximation holds or not depends on the characteristics of the internal wave field. We will discuss the coupled mode description of the scattering by internal waves in Sec. 3.4 and the comparison between adiabatic and coupled mode methods in Sec 3.5.

From Eq.(3.33), the acoustic intensity can be written as

$$
I=A \sum_{m} \sum_{n} \Psi_{m}\left(z_{s}\right) \Psi_{m}(r, z) \Psi_{n}\left(z_{s}\right) \Psi_{n}(r, z) \frac{e^{i\left[\int_{0}^{r} k_{r m}\left(r^{\prime}\right) d r^{\prime}-\int_{0}^{r} k_{r n}\left(r^{\prime}\right) d r^{\prime}\right]}}{\sqrt{\int_{0}^{r} k_{r m}\left(r^{\prime}\right) d r^{\prime} \int_{0}^{r} k_{r n}\left(r^{\prime}\right) d r^{\prime}}}
$$

where

$$
A \equiv \frac{1}{8 \pi \rho^{2}\left(z_{s}\right)}
$$

Rearranging the terms in Eq.(3.36), we obtain

$$
\begin{aligned}
I=A \sum_{m} \frac{\Psi_{m}^{2}\left(z_{s}\right) \Psi_{m}^{2}(r, z)}{\int_{0}^{r} k_{r m}\left(r^{\prime}\right) d r^{\prime}} \\
\\
\quad+A \sum_{m} \sum_{n \neq m} \Psi_{m}\left(z_{s}\right) \Psi_{m}(r, z) \Psi_{n}\left(z_{s}\right) \Psi_{n}(r, z) \frac{e^{i\left[\int_{0}^{r} k_{r m}\left(r^{\prime}\right) d r^{\prime}-\int_{0}^{r} k_{r n}\left(r^{\prime}\right) d r^{\prime}\right]}}{\sqrt{\int_{0}^{r} k_{r m}\left(r^{\prime}\right) d r^{\prime} \int_{0}^{r} k_{r n}\left(r^{\prime}\right) d r^{\prime}}}
\end{aligned}
$$


The first term in the right side of Eq.(3.38) is the incoherent intensity and the second term is the interference term. In this section, we will use the incoherent acoustic intensity and transmission loss to examine the acoustic scattering by the internal waves. We will also discuss the coherent intensity, which is the sum of the incoherent intensity and the interference term as in Eq.(3.38), and compare between coherent and incoherent intensities. There are three reasons why we choose to evaluate incoherent intensity and transmission loss. First, as I will also discuss later in this thesis, the internal wave -induced fluctuations are random so that the statistical mean of the coherent intensity approaches the incoherent intensity under the condition of large variance. Secondly, as stated in the last section, for shallow water problems, the incoherent transmission loss is often appropriate since bottom properties are often poorly known, and hence the detailed interference pattern predicted by coherent transmission loss calculation is not always correct. Finally, it is easier to analytically keep track of the derivations of the theoretical equations, so we start with the simple case first.

The incoherent acoustic intensity can be expressed as

$$
I_{i n c}=A \sum_{m=1}^{\infty} \frac{\left|\Psi_{m}\left(z_{s}\right) \Psi_{m}(r, z)\right|^{2}}{\int_{0}^{r} k_{r m}\left(r^{\prime}\right) d r^{\prime}}
$$

Next, we will use perturbation methods to evaluate $I_{\text {inc }}$. The perturbed sound speed is

$$
c(r, z)=c_{0}(r, z)+\delta c(r, z)
$$

where $\delta c(r, z)$ is the sound speed fluctuation, and $c_{0}(r, z)$ is the background sound speed profile. $\delta c(r, z)$ is related to the internal waves by

$$
\delta c(r, z)=S_{z}(r, z) \xi(r, z)
$$


where $S_{z}(r, z)=\frac{\partial}{\partial z} c_{0}(r, z)$ is the sound speed gradient in the $z$ direction and $\xi(r, z)$ is vertical displacement in water column, which can be expressed as

$$
\xi(r, z)=\sum_{n} a_{n}(r) \phi_{n}(r, z)
$$

where the $\phi_{n}(r, z)$ 's are the internal wave modes and the $a_{n}(r)$ 's are their amplitudes. The $a_{n}(r)$ 's are random variables whose mean and variance are

$$
\begin{gathered}
E\left(a_{n}\right)=0 \\
\operatorname{Var}\left[a_{n}\right]=E\left[a_{n}^{2}\right]
\end{gathered}
$$

From the above, we have

$$
E[\delta c]=0 .
$$

Since $\delta c \ll c_{0}$, we can use perturbation methods to evaluate the acoustic wavenumber and mode function perturbations about the background. According to the paper by Rajan et al[22], the acoustic horizontal wavenumber perturbation due to $\delta c$ is

$$
\delta k_{r m}(r)=\frac{-1}{k_{r m}^{(0)}} \int_{0}^{\infty} \rho_{0}^{-1}\left|\Psi_{m}^{(0)}\right|^{2} k_{0}^{2} \frac{\delta c}{c_{0}} d z
$$

where subscripts 0 and superscript (0) represent unperturbed terms. So we have

$$
k_{r m}(r)=k_{r m}^{(0)}+\delta k_{r m}(r)
$$

Now we will find the mode function perturbations due to $\delta c$, i.e. evaluate $\delta \Psi_{m}(r, z)$ in

$$
\Psi_{m}(r, z)=\Psi_{m}^{(0)}(z)+\delta \Psi_{m}(r, z)
$$

From a derivation using perturbation methods (Appendix A), we have 


$$
\delta \Psi_{m}(r, z)=\sum_{n \neq m} a_{m n}(r) \Psi_{n}^{(0)}(z)
$$

where

$$
a_{m n}(r)=\frac{\int 2 k_{0} \delta k \Psi_{m}^{(0)} \Psi_{n}^{(0)} d z}{k_{r m}^{(0) 2}-k_{r n}^{(0) 2}}
$$

Since $k=\frac{\omega}{c}$, then $\delta k=-\frac{\omega}{c_{0}^{2}} \delta c$. Substituting into Eq.(3.50) and then into Eq.(3.49) gives us

$$
\delta \Psi_{m}(r, z)=\sum_{n \neq m} \frac{\int 2 k_{0}\left(-\frac{\omega}{c_{0}^{2}} \delta c\right) \Psi_{m}^{(0)} \Psi_{n}^{(0)} d z}{k_{r m}^{(0) 2}-k_{r n}^{(0) 2}} \Psi_{n}^{(0)}(z)
$$

Now we can expand Eq. (3.39) into

$$
I_{i n c}(r, z)=A \sum_{m=1}^{\infty} \frac{\left[\Psi_{m}^{(0)}\left(0, z_{s}\right)+\delta \Psi_{m}\left(0, z_{s}\right)\right]^{2}\left[\Psi_{m}^{(0)}(r, z)+\delta \Psi_{m}(r, z)\right]^{2}}{\int_{0}^{r}\left[k_{r m}^{(0)}+\delta k_{r m}\left(r^{\prime}\right)\right] d r^{\prime}}
$$

To the first order, this can be further simplified. For the mode perturbation terms,

$$
\begin{gathered}
{\left[\Psi_{m}^{(0)}\left(0, z_{s}\right)+\delta \Psi_{m}\left(0, z_{s}\right)\right]^{2}\left[\Psi_{m}^{(0)}(r, z)+\delta \Psi_{m}(r, z)\right]^{2}} \\
=\left[\Psi_{m}^{(0) 2}\left(0, z_{s}\right)+2 \delta \Psi_{m}\left(0, z_{s}\right) \Psi_{m}^{(0)}\left(0, z_{s}\right)\right]\left[\Psi_{m}^{(0) 2}(r, z)+2 \delta \Psi_{m}(r, z) \Psi_{m}^{(0)}(r, z)\right] \\
=\Psi_{m}^{(0) 2}\left(0, z_{s}\right) \Psi_{m}^{(0) 2}(r, z)+2 \delta \Psi_{m}\left(0, z_{s}\right) \Psi_{m}^{(0)}\left(0, z_{s}\right) \Psi_{m}^{(0) 2}(r, z)+2 \delta \Psi_{m}(r, z) \Psi_{m}^{(0)}(r, z) \Psi_{m}^{(0) 2}\left(0, z_{s}\right)
\end{gathered}
$$

And for the wavenumber perturbation term,

$$
\begin{gathered}
\frac{1}{\int_{0}^{r}\left[k_{r m}^{(0)}+\delta k_{r m}\left(r^{\prime}\right)\right] d r^{\prime}}=\frac{1}{\int_{0}^{r} k_{r m}^{(0)} d r^{\prime}+\int_{0}^{r} \delta k_{r m}\left(r^{\prime}\right) d r^{\prime \prime}} \\
=\frac{1}{\int_{0}^{r} k_{r m}^{(0)}\left(r^{\prime}\right) d r^{\prime}}\left[1-\frac{\int_{0}^{r} \delta k_{r m}\left(r^{\prime}\right) d r^{\prime}}{\int_{0}^{r} k_{r m}^{(0)}\left(r^{\prime}\right) d r^{\prime}}\right]
\end{gathered}
$$


Substituting $\mathrm{Eq}(3.53)$ and $\mathrm{Eq}(3.54)$ into $\mathrm{Eq}(3.52)$ and further simplifying it to first order gives us

$$
I_{i n c}(r, z)=I_{i n c}^{(0)}(r, z)+\delta I_{i n c}^{(1)}(r, z)+\delta I_{i n c}^{(2)}(r, z)
$$

where

$$
I_{\text {inc }}^{(0)}(r, z)=A \sum_{m=1}^{\infty} \frac{1}{\int_{0}^{r} k_{r m}^{(0)}\left(r^{\prime}\right) d r^{\prime}} \Psi_{m}^{(0) 2}\left(0, z_{s}\right) \Psi_{m}^{(0) 2}(r, z)
$$

is the intensity without any internal wave-induced perturbations, and

$$
\begin{aligned}
& \delta I_{i n c}^{(1)}(r, z)=A \\
& \sum_{m=1}^{\infty} \frac{1}{\int_{0}^{r} k_{r m}^{(0)}\left(r^{\prime}\right) d r^{\prime}}\left[2 \delta \Psi_{m}\left(0, z_{s}\right) \Psi_{m}^{(0)}\left(0, z_{s}\right) \Psi_{m}^{(0) 2}(r, z)+2 \delta \Psi_{m}(r, z) \Psi_{m}^{(0)}(r, z) \Psi_{m}^{(0) 2}\left(0, z_{s}\right)\right]
\end{aligned}
$$

is the intensity fluctuation due to mode shape perturbations, and

$$
\delta I_{\text {inc }}^{(2)}(r, z)=A \sum_{m=1}^{\infty} \frac{1}{\int_{0}^{r} k_{r m}^{(0)}\left(r^{\prime}\right) d r^{\prime}} \frac{\int_{0}^{r} \delta k_{r m}\left(r^{\prime}\right) d r^{\prime}}{\int_{0}^{r} k_{r m}^{(0)}\left(r^{\prime}\right) d r^{\prime}} \Psi_{m}^{(0) 2}\left(0, z_{s}\right) \Psi_{m}^{(0) 2}(r, z)
$$

is the intensity fluctuation due to horizontal wavenumber perturbations.

From Eq.(3.46) and Eq.(3.51), the eigenvalue and eigenfunction perturbations are both caused by the internal wave-induced sound speed fluctuation and the mean value of the perturbation should be zero, i.e.

$$
E\left[\delta I_{i n c}^{(1)}(r, z)\right]=0
$$

and

$$
E\left[\delta I_{i n c}^{(2)}(r, z)\right]=0
$$


because of $E[\delta c]=0$.

Before evaluating the variance, we first make some simplifications to Eq.(3.57) and Eq.(3.58). We assume that the background sound speed profile is range-independent. Then the eigenvalue $k_{r m}^{(0)}$ and eigenfunction $\Psi_{m}^{(0)}(z)$ are range-independent. Thus Eq.(3.57) and Eq.(3.58) can be simplified to

$\delta I_{\text {inc }}^{(1)}(r, z)$

$$
=A \sum_{m=1}^{\infty} \frac{1}{k_{r m}^{(0)} r}\left[2 \delta \Psi_{m}\left(0, z_{s}\right) \Psi_{m}^{(0)}\left(z_{s}\right) \Psi_{m}^{(0) 2}(z)+2 \delta \Psi_{m}(r, z) \Psi_{m}^{(0)}(z) \Psi_{m}^{(0) 2}\left(z_{s}\right)\right]
$$

and

$$
\delta I_{i n c}^{(2)}(r, z)=A \sum_{m=1}^{\infty} \frac{1}{k_{r m}^{(0)} r} \frac{\int_{0}^{r} \delta k_{r m}\left(r^{\prime}\right) d r^{\prime}}{k_{r m}^{(0)} r} \Psi_{m}^{(0) 2}\left(z_{s}\right) \Psi_{m}^{(0) 2}(z)
$$

From Eq.(3.59) and Eq.(3.60), we know that

$$
E\left[I_{\text {inc }}\right]=I_{\text {inc }}^{(0)}
$$

Then the variance of intensity is

$$
\operatorname{Var}\left[I_{i n c}\right]=E\left[\left(\delta I_{i n c}^{(1)}+\delta I_{i n c}^{(2)}\right)^{2}\right]=E\left[\left(\delta I_{i n c}^{(1)}\right)^{2}\right]+2 E\left[\delta I_{i n c}^{(1)} \delta I_{i n c}^{(2)}\right]+E\left[\left(\delta I_{i n c}^{(2)}\right)^{2}\right]
$$

Before we start evaluating the variance, let's examine Eqs. (3.61) and (3.62). In Eq.(3.62), we notice the $1 / \mathrm{r}$ scaling factor which would cause $\delta I_{\text {inc }}^{(2)}$ to fall off quickly with range with respect to $\delta I_{i n c}^{(1)}$. So we can omit $\delta I_{i n c}^{(2)}$ in the intensity perturbation calculations from now on. Thus the only significant term on the right hand side of Eq.(3.64) is the first term which can be written as 


$$
E\left[\left(\delta l_{i n c}^{(1)}\right)^{2}\right]=E\left[A^{2} \sum_{m} \frac{1}{k_{r m}^{(0)} r}\left[G_{m}+J_{m}\right] \sum_{n} \frac{1}{k_{r n}^{(0)} r}\left[G_{n}+J_{n}\right]\right]
$$

where

$$
G_{m} \equiv 2 \delta \Psi_{m}\left(0, z_{s}\right) \Psi_{m}^{(0)}\left(z_{s}\right) \Psi_{m}^{(0) 2}(z)
$$

and

$$
J_{m} \equiv 2 \delta \Psi_{m}(r, z) \Psi_{m}^{(0)}(z) \Psi_{m}^{(0) 2}\left(z_{s}\right)
$$

So we have

$$
E\left[\left(\delta l_{i n c}^{(1)}\right)^{2}\right]=A^{2} \sum_{m} \sum_{n} \frac{1}{k_{r m}^{(0)} r} \frac{1}{k_{r n}^{(0)} r}\left[E\left[G_{m} G_{n}\right]+E\left[G_{m} J_{n}\right]+E\left[J_{m} G_{n}\right]+E\left[J_{m} J_{n}\right]\right]
$$

where

$$
\begin{aligned}
E\left[G_{m} G_{n}\right] & =4 \Psi_{m}^{(0)}\left(z_{s}\right) \Psi_{n}^{(0)}\left(z_{s}\right) \Psi_{m}^{(0) 2}(z) \Psi_{n}^{(0) 2}(z) E\left[\delta \Psi_{m}\left(0, z_{s}\right) \delta \Psi_{n}\left(0, z_{s}\right)\right], \\
E\left[G_{m} J_{n}\right] & =4 \Psi_{m}^{(0)}\left(z_{s}\right) \Psi_{n}^{(0)}(z) \Psi_{m}^{(0) 2}(z) \Psi_{n}^{(0) 2}\left(z_{s}\right) E\left[\delta \Psi_{m}\left(0, z_{s}\right) \delta \Psi_{n}(r, z)\right], \\
E\left[J_{m} G_{n}\right] & =4 \Psi_{m}^{(0)}(z) \Psi_{n}^{(0)}\left(z_{s}\right) \Psi_{m}^{(0) 2}\left(z_{s}\right) \Psi_{n}^{(0) 2}(z) E\left[\delta \Psi_{m}(r, z) \delta \Psi_{n}\left(0, z_{s}\right)\right], \\
E\left[J_{m} J_{n}\right] & =4 \Psi_{m}^{(0)}(z) \Psi_{n}^{(0)}(z) \Psi_{m}^{(0) 2}\left(z_{s}\right) \Psi_{n}^{(0) 2}\left(z_{s}\right) E\left[\delta \Psi_{m}(r, z) \delta \Psi_{n}(r, z)\right] .
\end{aligned}
$$

By substituting (3.51) into (3.69) - (3.72), we obtain

$E\left[\delta \Psi_{m}\left(0, z_{s}\right) \delta \Psi_{n}\left(0, z_{s}\right)\right]$

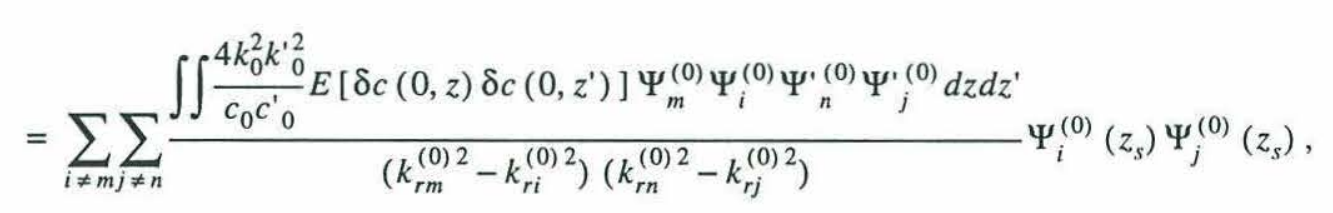

$E\left[\delta \Psi_{m}\left(0, z_{s}\right) \delta \Psi_{n}(r, z)\right]$

$$
=\sum_{i \neq m} \sum_{j \neq n} \frac{\iint_{0}^{4 k_{0}^{2} k_{0}^{\prime 2}} \frac{c_{0} c_{0}^{\prime}}{c_{0}} E\left[\delta c(0, z) \delta c\left(r, z^{\prime}\right)\right] \Psi_{m}^{(0)} \Psi_{i}^{(0)} \Psi_{n}^{\prime(0)} \Psi_{j}^{\prime(0)} d z d z^{\prime}}{\left(k_{r m}^{(0) 2}-k_{r i}^{(0) 2}\right)\left(k_{r n}^{(0) 2}-k_{r j}^{(0) 2}\right)} \Psi_{i}^{(0)}\left(z_{s}\right) \Psi_{j}^{(0)}(z)
$$


$E\left[\delta \Psi_{m}(r, z) \delta \Psi_{n}\left(0, z_{s}\right)\right]$

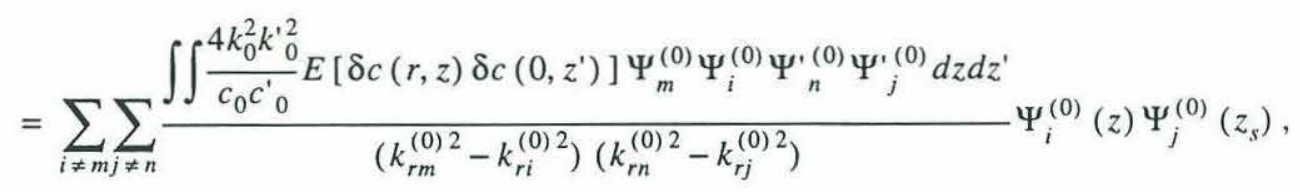

and

$E\left[\delta \Psi_{m}(r, z) \delta \Psi_{n}(r, z)\right]$

$$
=\sum_{i \neq m} \sum_{j \neq n} \frac{\iint \frac{4 k_{0}^{2} k_{0}^{\prime 2}}{c_{0} c_{0}^{\prime}} E\left[\delta c(r, z) \delta c\left(r, z^{\prime}\right)\right] \Psi_{m}^{(0)} \Psi_{i}^{(0)} \Psi_{n}^{\prime(0)} \Psi_{j}^{\prime(0)} d z d z^{\prime}}{\left(k_{r m}^{(0) 2}-k_{r i}^{(0) 2}\right)\left(k_{r n}^{(0) 2}-k_{r j}^{(0) 2}\right)} \Psi_{i}^{(0)}(z) \Psi_{j}^{(0)}(z)
$$

If we assume $\delta c$ is stationary over range and depth, we have

$$
E\left[\delta c(r, z) \delta c\left(r^{\prime}, z^{\prime}\right)\right]=R_{\delta c}\left(r-r^{\prime}, z-z^{\prime}\right)
$$

which is the autocorrelation function of $\delta c$. With Eq.(3.41), we can reduce Eq.(3.77) to

$$
E\left[\delta c(r, z) \delta c\left(r^{\prime}, z^{\prime}\right)\right]=S_{z} S^{\prime}{ }_{z} E\left[\xi(r, z) \xi\left(r^{\prime}, z^{\prime}\right)\right]=S_{z} S_{z}{ }_{z} R_{\xi}\left(r-r^{\prime}, z-z^{\prime}\right)
$$

where $R_{\xi}\left(r-r^{\prime}, z-z^{\prime}\right)$ is the 2-D autocorrelation function of vertical displacement $\xi$. The autocorrelation function is related to the horizontal and vertical wavenumber spectrum $S_{\xi}\left(k_{r}, k_{z}\right)$ by Fourier transform:

$$
R_{\xi}(r, z)=\int S_{\xi}\left(k_{r}, k_{z}\right) \exp \left[i\left(k_{r} r+k_{z} z\right)\right] d k_{r} d k_{z} .
$$

With Eqs. (3.68) - (3.79), we can evaluate the intensity variance in Eq.(3.64). Furthermore, we assume that the 2-D correlation function can be factored into or at least approximated by the product of horizontal autocorrelation function $R_{r}(r)$ and vertical autocorrelation function $R_{z}(z)$, i.e. 


$$
R_{\xi}(r, z)=R_{\xi r}(r) R_{\xi z}(z) .
$$

Substituting Eqs. (3.77), (3.78) and (3.80) into Eqs. (3.73)-(3.76) and manipulating the terms, we obtain

$$
\begin{aligned}
E\left[\delta \Psi_{m}\left(0, z_{s}\right) \delta \Psi_{n}\left(0, z_{s}\right)\right] & =R_{\xi r}(0) \sum_{i \neq m \neq \neq n} \sum_{m n i j} \Psi_{i}^{(0)}\left(z_{s}\right) \Psi_{j}^{(0)}\left(z_{s}\right) \\
E\left[\delta \Psi_{m}\left(0, z_{s}\right) \delta \Psi_{n}(r, z)\right] & =R_{\xi r}(r) \sum_{i \neq m} \sum_{j \neq n} K_{m n i j} \Psi_{i}^{(0)}\left(z_{s}\right) \Psi_{j}^{(0)}(z) \\
E\left[\delta \Psi_{m}(r, z) \delta \Psi_{n}\left(0, z_{s}\right)\right] & =R_{\xi r}(r) \sum_{i \neq m j \neq n} K_{m n i j} \Psi_{i}^{(0)}(z) \Psi_{j}^{(0)}\left(z_{s}\right) \\
E\left[\delta \Psi_{m}(r, z) \delta \Psi_{n}(r, z)\right] & =R_{\xi r}(0) \sum_{i \neq m} \sum_{j \neq n} K_{m n i j} \Psi_{i}^{(0)}(z) \Psi_{j}^{(0)}(z)
\end{aligned}
$$

where

$$
K_{m n i j} \equiv \frac{\iint \frac{4 k_{0}^{2} k_{0}^{\prime 2}}{c_{0} c_{0}^{\prime}} R_{\xi z}\left(z-z^{\prime}\right) S_{z} S_{z}^{\prime} \Psi_{m}^{(0)} \Psi_{i}^{(0)} \Psi_{n}^{\prime(0)} \Psi_{j}^{\prime(0)} d z d z^{\prime}}{\left(k_{r m}^{(0) 2}-k_{r i}^{(0) 2}\right)\left(k_{r n}^{(0) 2}-k_{r j}^{(0) 2}\right)}
$$

Eqs.(3.81)-(3.84) show that the horizontal structure of the incoherent intensity variance is determined by the autocorrelation function of the vertical displacement, i.e. $R_{\xi r}(r)$.

After finishing the incoherent intensity derivations, we now look at Eq.(3.38) to examine coherent intensity. According to Eq.(3.38), the coherent intensity is the sum of the incoherent intensity and the interference terms. Thus the coherent intensity fluctuation is the sum of the incoherent intensity fluctuation and fluctuations of the interference terms. The interference term fluctuations are mainly caused by horizontally shifting of nulls in the interference structure of coherent intensity. We then need to determine the relative levels of the 'incoherent' fluctuations and 'interference' fluctuations. For the range-independent problem without any fluctuations, according to Eq.(3.38), we know that the 
incoherent term and the interference term contribute to the coherent intensity at the same level. For the case of fluctuations, the incoherent fluctuations are first order perturbations which are much smaller than the unperturbed incoherent intensity according to the above derivations However, the interference fluctuations are basically caused by modal phase fluctuations. Due to phase wrapping effects, the phase fluctuations can have strong effect on the interference structure by causing nulls to shift back and forth, and hence the coherent intensity. Thus the interference intensity fluctuations are at the same level as the interference intensity without fluctuations. With the above arguments, we can conclude that the coherent intensity fluctuations are mainly due to the interference fluctuations, i.e. the modal phase fluctuation. And then the horizontal structure, i.e. the range-dependence of the variance of the coherent intensity, is determined by the range-dependence of the variance of the modal phase fluctuation.

We now investigate the variance of the phase fluctuations. The modal phase is

$$
\varphi_{m}=\int_{0}^{r} k_{r m}\left(r^{\prime}\right) d r^{\prime}=k_{r m}^{(0)} r+\int_{0}^{r} \delta k_{r m}\left(r^{\prime}\right) d r^{\prime}
$$

And the variance of the phase is thus

$$
\operatorname{Var}\left[\varphi_{m}\right]=E\left[\int_{0}^{r} \delta k_{r m}\left(r^{\prime}\right) d r^{\prime} \int_{0}^{r} \delta k_{r m}\left(r^{\prime \prime}\right) d r^{\prime \prime}\right]
$$

Substituting Eq.(3.46) into it, we find that

$$
\operatorname{Var}\left[\varphi_{m}\right]=\frac{-1}{k_{r m}^{(0) 2}} \int_{0}^{\infty} \int_{0}^{\infty} \frac{1}{\rho_{0} \rho_{0}^{\prime}}\left|\Psi_{0}\right|^{2}\left|\Psi^{\prime}{ }_{0}\right|^{2} k_{0}^{2} k_{0}^{\prime 2} \frac{E\left[\int_{0}^{r} \int_{0}^{r} \delta c\left(r^{\prime}\right) \delta c^{\prime}\left(r^{\prime \prime}\right) d r^{\prime} d r^{\prime \prime}\right]}{c_{0} c^{\prime}{ }_{0}} d z d z^{\prime}
$$

Here the prime on the variable means that its argument is $z^{\prime}$. Now we want to explore the range-dependence of the variance. Looking at the terms on the right side of Eq.(3.88), we 
note that the range dependence is determined by the double integral $\int_{0}^{r} \int_{0}^{r} \delta c\left(r^{\prime}\right) \delta c^{\prime}\left(r^{\prime \prime}\right) d r^{\prime} d r^{\prime \prime}$, i.e.

$$
\operatorname{Var}\left[\varphi_{m}\right]-E\left[\int_{0}^{r} \int_{0}^{r} \delta c\left(r^{\prime}\right) \delta c^{\prime}\left(r^{\prime \prime}\right) d r^{\prime} d r^{\prime \prime}\right]
$$

Since the sound speed fluctuation $\delta c$ is related to the internal wave vertical displacement by Eq.(3.41), substituting it into Eq.(3.89) gives us

$$
\operatorname{Var}\left[\varphi_{m}\right] \sim E\left[\int_{0}^{r} \int_{0}^{r} \xi\left(r^{\prime}\right) \xi\left(r^{\prime \prime}\right) d r^{\prime} d r^{\prime \prime}\right]
$$

In the above step, we made some simplification by neglecting the depth dependence of $\xi$. (this will not alter the qualitative results we will obtain shortly). We next consider an internal wave at a single frequency. This doesn't lose generality because the spectrum can be decomposed into sinusoids by Fourier transform anyway. Thus we assume $\xi(r)$ is a stochastic process which takes the form of

$$
\xi(r)=\cos \left(k_{r} r+\theta\right)
$$

where $k_{r}$ is the internal wave wavenumber and $\theta$ is a random phase with uniform distribution, i.e.

$$
p_{\theta}(\Theta)=\frac{1}{2 \pi}, \Theta \in[0,2 \pi]
$$

which is the probability density function of $\theta$. Substituting Eq.(3.91) and Eq.(3.92) into Eq.(3.90) gives us

$$
\operatorname{Var}\left[\varphi_{m}\right] \sim \int_{0}^{2 \pi} \int_{0}^{r} \int_{0}^{r} \cos \left(k_{r} r^{\prime}+\Theta\right) \cos \left(k_{r} r^{\prime \prime}+\Theta\right) \frac{1}{2 \pi} d r^{\prime} d r^{\prime \prime} d \Theta
$$


The integral on the right side can be easily evaluated and the result is

$$
\operatorname{Var}\left[\varphi_{m}\right] \sim \frac{1}{k_{r}^{2}} \sin ^{2}\left(\frac{k_{r} r}{2}\right)
$$

Thus the variance of the modal phase is periodical over range with the same wavelength as the internal wave. Eq.(3.94) also shows that the variance starts from zero and goes back to zero at the end of an internal wave cycle. Based on previous discussions, we can predict that the variance of the coherent intensity fluctuations has the same horizontal structure, i.e. the coherent intensity variance starts at the source with a very small value, which is actually the incoherent intensity fluctuation, increases with range up to the mid-point of the 'dominant' internal wave cycle, and then decreases with range until the end of the internal wave cycle to the same level as that at the source. In the next chapter, we will see that the numerical results agree with this predication very well.

\subsection{Coupled mode description of acoustic scattering by internal waves}

In this section we briefly investigate mode coupling effects due to the internal waves. The

derivations follow the work of S. T. McDaniel [23]. To begin with, the mode coupling equation is

$$
\left(\nabla_{\perp}^{2}+k_{n}^{2}\right) \Psi_{n}=-\sum_{m \neq n}\left(B_{m n}+C_{m n} \nabla_{\perp}\right) \Psi_{m}
$$

where

$$
B_{m n}=-\int \Psi_{n} \nabla_{\perp}^{2} \Psi_{m} d z
$$

and

$$
C_{m n}=2 \int \Psi_{n} \nabla_{\perp} \Psi_{m} d z
$$


where $\Psi_{n}$ and $\Psi_{m}$ are normalized mode functions.

If a cylindrically symmetric geometry is assumed, and $P_{n}=\Psi_{n} r^{1 / 2}$ is substituted into Eq.(3.95), we find that for $k_{n} r » 1$,

$$
\left(\frac{d^{2}}{d r^{2}}+k_{n}^{2}\right) P_{n}=-\sum_{m \neq n}\left(B_{m n}+C_{m n} \frac{d}{d r}\right) P_{m}
$$

To solve Eq.(3.98), the field is split into a forward component characterized by propagation of the form $\exp \left(i k_{n} r\right)$ and a backscattered component:

$$
P_{n}=u_{n} \exp \left(i \bar{k}_{n} r\right)+v_{n} \exp \left(-i \bar{k}_{n} r\right)
$$

where $\overline{k_{n}} r=\int_{0}^{r} k_{n}\left(r^{\prime}\right) d r^{\prime}$.

After substitution and some manipulation, one obtains

$$
\frac{d u_{n}}{d r}=\sum_{m} \frac{1}{2} \exp \left(-i \overline{k_{n}} r\right)\left[M_{m n} u_{m} \exp \left(i \overline{k_{m}} r\right)+Q_{m n} v_{m} \exp \left(-i \overline{k_{m}} r\right)\right]
$$

and

$$
\frac{d v_{n}}{d r}=\sum_{m} \frac{1}{2} \exp \left(i \bar{k}_{n} r\right)\left[M_{m n} u_{m} \exp \left(i \overline{k_{m}} r\right)+Q_{m n} v_{m} \exp \left(-i \overline{k_{m}} r\right)\right]
$$

where the matrices $M_{m n}$ and $Q_{m n}$ are given by

$$
\begin{gathered}
M_{m n}=-\delta_{m n} k_{n}^{-1} \frac{d k_{n}}{d r}+i k_{n}^{-1} B_{m n}-\frac{k_{m}}{k_{n}} C_{m n} \\
Q_{m n}=\delta_{m n} k_{n}^{-1} \frac{d k_{n}}{d r}+i k_{n}^{-1} B_{m n}+\frac{k_{m}}{k_{n}} C_{m n}
\end{gathered}
$$

If the backscattered field $v_{m}$ is neglected, Eq.(3.100) becomes

$$
\frac{d u_{n}}{d r}=\sum_{m} \frac{1}{2}\left[M_{m n} u_{m} \exp \left(i\left(\overline{k_{m}}-\overline{k_{n}}\right) r\right)\right]
$$


Now we will consider only forward propagation, and approximate

$$
M_{m n}=-\overline{C_{m n}}
$$

where $C_{m n}$ has been symmetrized:

$$
\overline{C_{m n}}=\frac{1}{2}\left(C_{m n}-C_{n m}\right)
$$

With this approximation, propagation loss computed using the coupled-mode equations will obey the reciprocity principle and energy will be conserved in the absence of absorption. The set of equations to be solved is then

$$
\frac{d u_{n}}{d r}=\sum_{m} \frac{1}{2}\left[\overline{C_{m n}} u_{m} \exp \left(i\left(\overline{k_{m}}-\overline{k_{n}}\right) r\right)\right]
$$

Before we solve this equation, we first find $\overline{C_{m n}}$ :

$$
\overline{C_{m n}}=\int \frac{\partial \Psi_{m}}{\partial r} \Psi_{n} d z=\int\left[\frac{\partial}{\partial r}\left(\Psi_{m}^{(0)}+\delta \Psi_{m}\right)\right]\left(\Psi_{n}^{(0)}+\delta \Psi_{n}\right) d z
$$

Suppose the unperturbed terms are range-independent(.i.e we only consider internal wave introduced range-dependence), then we have $\frac{\partial \Psi^{(0)}}{\partial r}=0$. Substituting this into Eq.(3.108), and retaining terms to the first order, we obtain

$$
\overline{C_{m n}}=\int \Psi_{n}^{(0)} \frac{\partial}{\partial r}\left(\delta \Psi_{m}\right) d z
$$

According to Eq.(A.19) and Eq.(A.20), we have

$$
\frac{\partial}{\partial r} \delta \Psi_{m}=\frac{\partial}{\partial r} \sum_{j \neq m} a_{m j} \Psi_{j}^{(0)}=\sum_{j \neq m} \frac{\partial a_{m j}}{\partial r} \Psi_{j}^{(0)}
$$

and 


$$
\begin{aligned}
& \frac{\partial a_{m j}}{\partial r}=\frac{\partial}{\partial r}\left[\int \frac{2 k_{0} \frac{-\omega}{c_{0}^{2}} \delta c}{k_{r m}^{(0) 2}-k_{r j}^{(0) 2}} \Psi_{m}^{(0)} \Psi_{j}^{(0)} d z\right] \\
& =\frac{\int 2 k_{0} \frac{-\omega}{c_{0}^{2}}\left(\frac{\partial}{\partial r} \delta c\right) \Psi_{m}^{(0)} \Psi_{j}^{(0)} d z}{k_{r m}^{(0) 2}-k_{r j}^{(0) 2}}
\end{aligned}
$$

Now we can evaluate $u_{n}$ from Eq.(3.107). With no loss of generality, we only consider the coupling between mode $m$ and mode $n$. Integrating Eq.(3.107) over range $r$ gives us

$$
u_{n}(r)=\int_{0}^{r} \frac{1}{2}\left[\overline{C_{m n}} u_{m} \exp \left(i\left(\overline{k_{m}}-\overline{k_{n}}\right) r^{\prime}\right)\right] d r^{\prime}
$$

Substituting Eqs.(3.109), (3.110) and (3.111) into Eq.(3.112), assuming weak coupling (i.e. $u_{m}$ is almost constant over range), we find that

$$
u_{n}(r) \sim \int_{0}^{r}\left(\frac{\partial}{\partial r^{\prime}} \delta c\right) \exp \left(i\left(\overline{k_{m}}-\overline{k_{n}}\right) r^{\prime}\right) d r^{\prime}
$$

Now we consider the internal wave at a single wavenumber $k_{\xi r}$, i.e

$$
\xi(r)=S\left(k_{\xi_{r}}\right) e^{i k_{\xi, r}} .
$$

Substituting Eq.(3.41) and Eq.(3.114) into Eq.(3.113) gives us

$$
u_{n}(r) \sim k_{\xi} S\left(k_{\xi r}\right) \int_{0}^{r} e^{i\left(\bar{k}_{m}-\bar{k}_{n}+k_{\xi}\right) r^{\prime}} d r^{\prime} .
$$

We see that "Bragg scattering" occurs when

$$
k_{\xi_{r}}=\overline{k_{n}}-\overline{k_{m}} .
$$


The amplitude of the Bragg resonant term is seen to be proportional to the magnitude of the internal wave wavenumber spectrum at $k_{\xi r}$, i.e. $S\left(k_{\xi r}\right)$. Before we evaluate this effect in more detail, we consider the more general case in which $\delta c$ is a random variable. Then,

$$
E\left[\left|u_{n}\right|^{2}\right] \sim E\left[\int_{0}^{R}\left(\frac{\partial}{\partial r} \delta c\right) e^{i\left(\overline{k_{m}}-\overline{k_{n}}\right) r} d r \int_{0}^{R}\left(\frac{\partial}{\partial r^{\prime}} \delta c\right) e^{-i\left(\overline{k_{m}}-\bar{k}_{n}\right) r^{\prime}} d r^{\prime}\right]
$$

Substituting Eq.(3.41) into the above equation and manipulating the terms, we then have

$$
E\left[\left|u_{n}\right|^{2}\right] \sim \int_{0}^{R} \int_{0}^{R} \frac{\partial^{2}}{\partial r \partial r^{\prime}} E\left[\xi(r) \xi\left(r^{\prime}\right)\right] e^{i\left(\overline{k_{m}}-\overline{k_{n}}\right)\left(r-r^{\prime}\right)} d r d r^{\prime}
$$

If the internal wave field is stationary over range, then we have

$$
E\left[\xi(r) \xi\left(r^{\prime}\right)\right]=R_{\xi}\left(r-r^{\prime}\right)
$$

which is the autocorrelation function of $\xi$. Substituting this into Eq.(3.118) gives us

$$
E\left[\left|u_{n}\right|^{2}\right]-\int_{0}^{R} \int_{0}^{R} \frac{\partial^{2}}{\partial r \partial r^{\prime}} R_{\xi}\left(r-r^{\prime}\right) e^{i\left(\bar{k}_{m}-\bar{k}_{n}\right)\left(r-r^{\prime}\right)} d r d r^{\prime}
$$

After changing the variables with $l=r-r^{\prime}$, we obtain

$$
E\left[\left|u_{n}\right|^{2}\right] \sim R \int_{-R}^{R} \frac{\partial^{2}}{\partial l^{2}} R_{\xi}(l) e^{i\left(\bar{k}_{m}-\bar{k}_{n}\right) l} d l
$$

The autocorrelation function is related to the power spectrum by a Fourier transform:

$$
R_{\xi}(l)=\int_{-\infty}^{\infty} S_{\xi}(k) e^{i k l} d k
$$

Substituting Eq.(3.122) into Eq.(3.121) gives us 


$$
E\left[\left|u_{n}\right|^{2}\right] \sim R \int_{-R}^{R} \frac{\partial^{2}}{\partial l^{2}}\left[\int_{-\infty}^{\infty} S_{\xi}(k) e^{i k l} d k\right] e^{i\left(\overline{k_{m}}-\bar{k}_{n}\right) l} d l
$$

Changing the integration order and expanding the derivatives, we have

$$
E\left[\left|u_{n}\right|^{2}\right] \sim R \int_{-\infty}^{\infty} k^{2} S_{\xi}(k) d k \int_{-R}^{R} e^{i\left(k+\bar{k}_{m}-\bar{k}_{n}\right) l} d l
$$

i.e.

$$
E\left[\left|u_{n}\right|^{2}\right] \sim R \int_{-\infty}^{\infty} k^{2} S_{\xi}(k) \operatorname{sinc}\left[\left(k+\overline{k_{m}}-\overline{k_{n}}\right) R\right] d k
$$

where $\operatorname{sinc}(\mathrm{x})=\frac{\sin x}{x}$. Also,

$$
\operatorname{sinc}\left[\left(k+\overline{k_{m}}-\overline{k_{n}}\right) R\right] \rightarrow \delta\left(k+\overline{k_{m}}-\overline{k_{n}}\right) \text { when }\left|k+\overline{k_{m}}-\overline{k_{n}}\right| \ll \frac{2 \pi}{R}
$$

When this condition holds, i.e. near resonance, substituting it into Eq.(3.125) gives us

$$
E\left[\left|u_{n}\right|^{2}\right] \sim R \int_{-\infty}^{\infty} k^{2} S_{\xi}(k) \delta\left(k+\overline{k_{m}}-\overline{k_{n}}\right) d k
$$

Thus we finally get the coupling effect result:

$$
E\left[\left|u_{n}\right|^{2}\right] \sim R\left(\overline{k_{m}}-\overline{k_{n}}\right)^{2} S_{\xi}\left(\overline{k_{n}}-\overline{k_{m}}\right)
$$

It is seen that: 1) the coupling effect is proportional to the magnitude of the internal wave spectrum at the wavenumber which is equal to the wavenumber difference between the two acoustic modes; 2) the coupling effects increase linearly with range. However, in the case of solitons, the coupling shows a somewhat different character. We discuss this in the next section. 


\subsection{Solitons vs. linear internal waves}

As seen in the last section, the scattering of the acoustic field by linear internal waves of a certain frequency is proportional to the energy in the internal wave spectrum at that frequency. As we discussed in Section 2.5, solitons are non-linear wave packets with welldefined wavelengths so that the energy of the solitons is contained in certain frequencies. So if the mode cycle distance of a given pair of acoustic modes happens to be equal to the soliton wavelength, resonance occurs, i.e. the coupling between the two modes is very strong. However, for linear internal waves as modeled by the Garrett-Munk spectrum, the internal wave spectrum is broadband with most energy contained in the low frequencies. Whether the acoustic mode coupling is strong or not depends on where the mode cycle distance falls in the internal wave spectrum. These two cases have been examined by Zhou et al. [5] and Lynch et al. [9], based on experimental results. Here we summarize their results and present some discussions.

Zhou and his group at the Institute of Acoustics, Chinese Academy of Sciences, measured the frequency response of shallow-water sound propagation in the situation of a strong thermocline in the Yellow Sea off the east coast of China. The measurement in the summer showed the transmission loss was strongly time dependent, anisotropic and sometimes exhibited an anomalously large attenuation over some frequency band. In order to explain these effects, based on the experimental measurements of sound speed profile and temperature, Zhou et al. established a simple three-layer ocean density structure model with solitons contained in the thermocline. He then used the PE (parabolic equation) method to numerically calculate the sound propagation loss through the modeled solitary internal wave field. The numerical results agreed with experimental propagation measurement very well. Most important, the calculations showed the anomalous frequency 
response observed in the experiment. By decomposing the PE generated field into normal modes, Zhou concluded that the anomalous frequency response was due to strong acoustic mode coupling caused by the interaction between acoustic waves and internal waves (the resonance effect discussed in the last section). The results were satisfactory except for the lack of any direct experimental evidence for the presence of solitons. Though sound speed profile measurements did show the thermocline depth changed with time and temperature records contained peaks which might be indications of individual solitary waves, no systematic measurements of the internal waves were done during those experiments. The authors also suggested that seabed roughness, sediment inhomogeneities and fish schools could produce similar acoustic attenuations over the experimental frequency band.

In the paper by Lynch et al., acoustic scattering by linear internal waves was investigated, in distinction to the scattering by solitons. The authors claimed that the adiabatic approximation could be accurate enough to describe the short-range acoustic scattering effects by linear internal waves under certain circumstances. Based on the data from the Barents Sea Polar Front experiment, simple calculations and order of magnitude estimation were made to show that the adiabatic approach was good enough for that case.

As we discussed, resonant mode coupling occurs when the internal wave wavelength matches the interference cycle distance of a given pair of acoustic modes. To estimate the cycle distances of the acoustic modes, a simple pressure release surface, isovelocity water column, and rigid bottom model can be used. For this simple model, we know that the mode cycle distance is

$$
\Delta_{m n}=\left|\frac{2 \pi}{k_{r m}-k_{r n}}\right|
$$

and hence 


$$
\Delta_{m n} \approx\left|\frac{4 k H^{2}}{n(n-1)-m(m-1)}\right| .
$$

We can see that the cycle distance increases linearly with acoustic frequency and quadratically with the waveguide depth, $\mathrm{H}$.

Since most of the internal wave energy is contained in the longest wavelengths (for linear internal waves) and the energy spectral density monotonically increases with wavelength, we should estimate the longest mode cycle distance and compare it with the internal wave wavelength. For the low order modes, the wavenumber spacings are generally the closest, thus giving the largest mode cycle distances. In the BSPF experiment the acoustic frequency was $224 \mathrm{~Hz}$ and the average water depth was $150 \mathrm{~m}$. Using our simple calculation, we see that $\Delta=9 \mathrm{~km}$, which is also the internal wavelength corresponding to the "maximum mode coupling". According to the dispersion relation showed in the Lynch et al paper, this wavelength corresponded to a 180 minute period, or an $8 \mathrm{cpd}$ frequency of the internal waves. From the internal wave spectrum obtained from BSPF experimental data analysis, it was seen that this frequency was an order of magnitude less energetic than the M2 peak for the standard GM spectrum, and a factor of 15 less energetic than the M2 peak in the measured data. Thus, the resonant mode coupling would have to amplify the scattering at $8 \mathrm{cpd}$ by nearly two orders of magnitude to compete with the purely adiabatic scattering at the M2 frequency. Thus the authors concluded that, "it seemed to us that for this case the energetics favored the adiabatic approach." As we will see in the next chapter, the same argument can be made for the case of a shallow water GM internal wave model. In general, we test this by comparing adiabatic and coupled mode calculations. 


\section{Chapter 4}

\section{Simulations and numerical calculations}

\subsection{Realizations of Garrett-Munk internal wave field}

As we discussed in Section 2.5, there are two different internal wave processes in the ocean: solitary internal waves and linear internal waves. Here we would like to concentrate on the amplitude scattering due to linear internal waves in shallow water. To describe the linear internal waves in shallow water, the Garrett-Munk model has been chosen. There are two main reasons why we chose the Garrett-Munk model: first, there are some shallow-water experimental data supporting an approximate Garrett-Munk model(e.g., Lynch et al.[9]); second, the GM model is a simple model to describe the general characteristics of linear internal waves in the ocean.

In describing the scattering, we first need to model the shallow water environment. In this thesis, we use a canonical three-layer model in which the top layer is a warm surface mixed layer with constant density and sound speed, the middle layer is the thermocline or pycnocline where the density and sound speed vary linearly over depth, and the bottom layer is the bottom boundary layer with constant density and sound speed. The buoyancy frequency profile $\mathrm{N}(\mathrm{z}$ ) corresponding to this situation for a 70m deep waveguide (a "typical" shelf depth) is depicted in Figure 4.1 (a). We need to know both the buoyancy frequency profile and the local inertial frequency $f$ to calculate the internal wave normal modes. Here we arbitrarily choose f for a latitude of 74 degrees, which is the latitude where the Barents Sea Polar Front experiment was conducted. Thus $f=\sin \left(74^{\circ}\right) / 12 \mathrm{cph}$ (cycles per hour). Use of a lower latitude would give a smaller $\mathrm{f}$, and thus make lower frequency internal waves possible. Given $\mathrm{N}$ and $\mathrm{f}$, we can calculate the IW modes from 
Eq.(2.15). We use simple finite-difference methods to calculate these modes. Since most of IW energy is contained in the lower modes, only modes 1-8 are used in the simulation of the IW field. The IW modes 1-8 are plotted in Figure 4.1 (b).

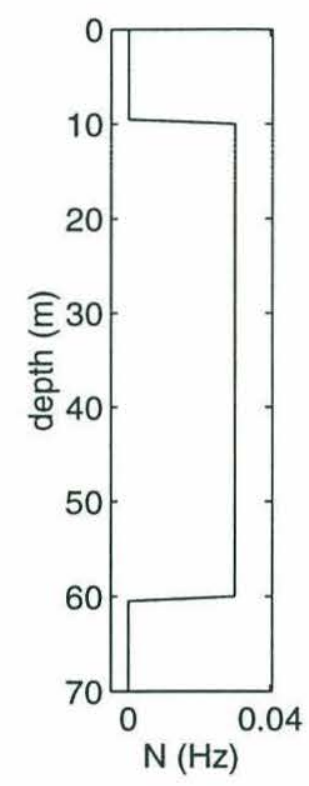

(a)

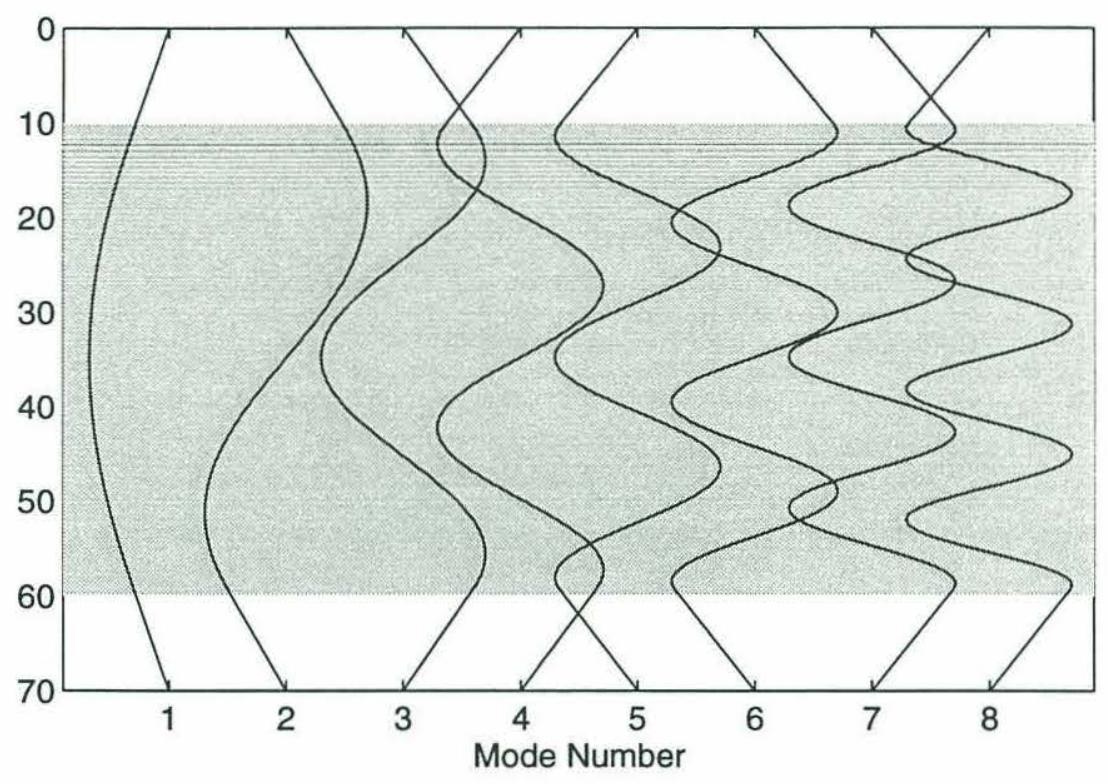

(b)

Figure 4.1: (a) Buoyancy frequency profile N(z); (b) Internal wave modes calculated from Eq.(2.15)

Given the IW modes, we next need to determine mode amplitudes to complete the IW field simulation. We use the GM spectrum, which has been described in Chapter 2, to provide the mode amplitudes. The GM frequency spectrum is plotted in Fig. 4.2. We then use the dispersion relation in Eq.(2.20) to map the frequency spectrum into a horizontal wavenumber spectrum, which is plotted in Fig.4.3. Since the spectrum only gives a magnitude 
at each wavenumber, we need to specify the phase. We use a phase which is a random variable with uniform distribution over $[0,2 \pi]$ to obtain realizations of the wavenumber spectrum. This spectrum is converted into mode amplitudes using inverse FFT methods. For each realization of the mode amplitudes, summing up the modes according to Eq.(2.19) gives us one realization of vertical isopycnal displacement for a 2-D IW field. Figure 4.4 shows a plot of one realization of the vertical displacement.

Using Eq.(3.41), we finally get the sound speed fluctuations due to the internal waves from the vertical displacement. Fig 4.5 shows one realization of sound speed fluctuations. We have thus completed the simulation of linear shallow water internal waves.

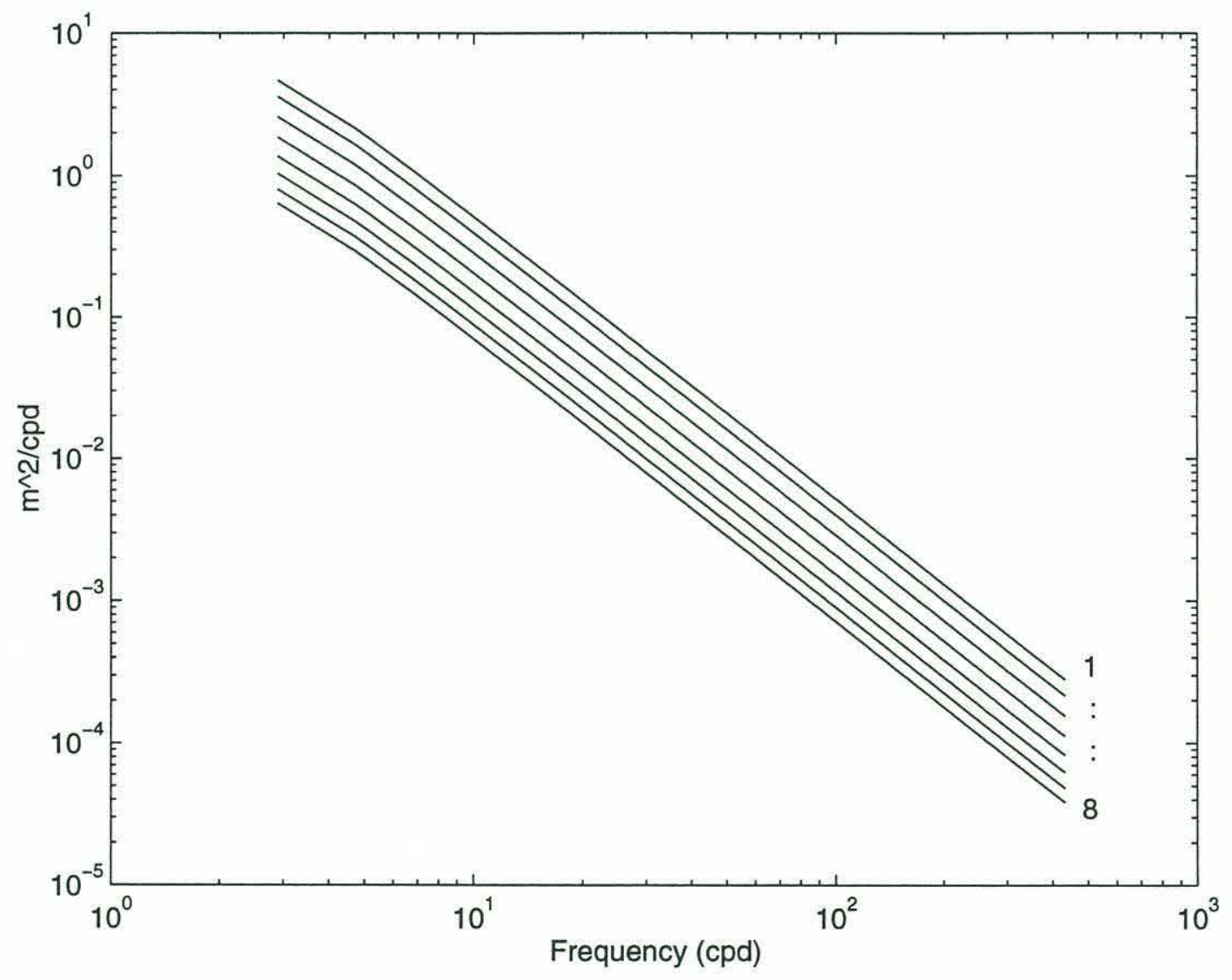

Figure 4.2: Garrett-Munk IW spectrum used for simulating the IW field for modes 1-8 


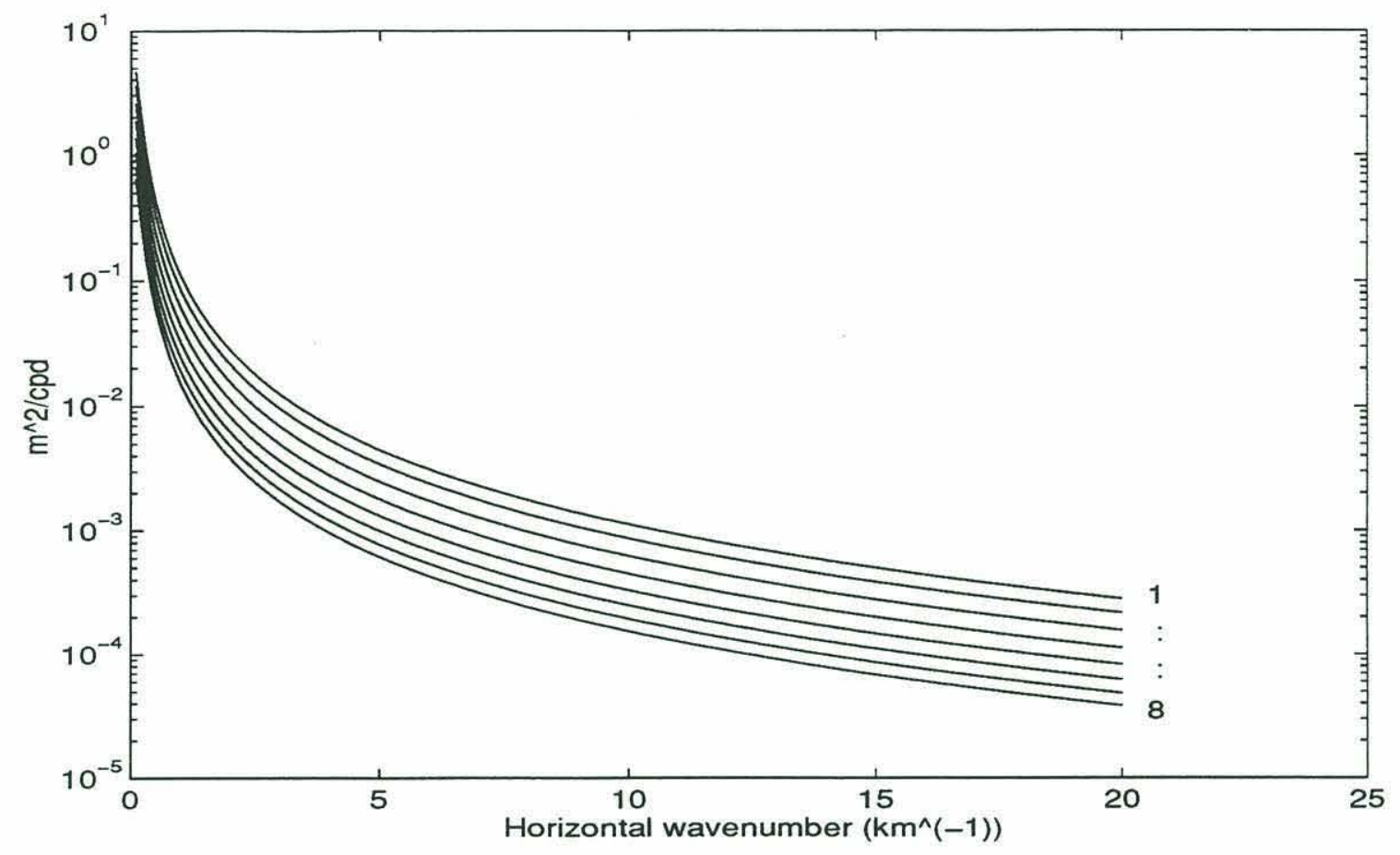

Figure 4.3: IW horizontal wavenumber spectrum for modes 1-8

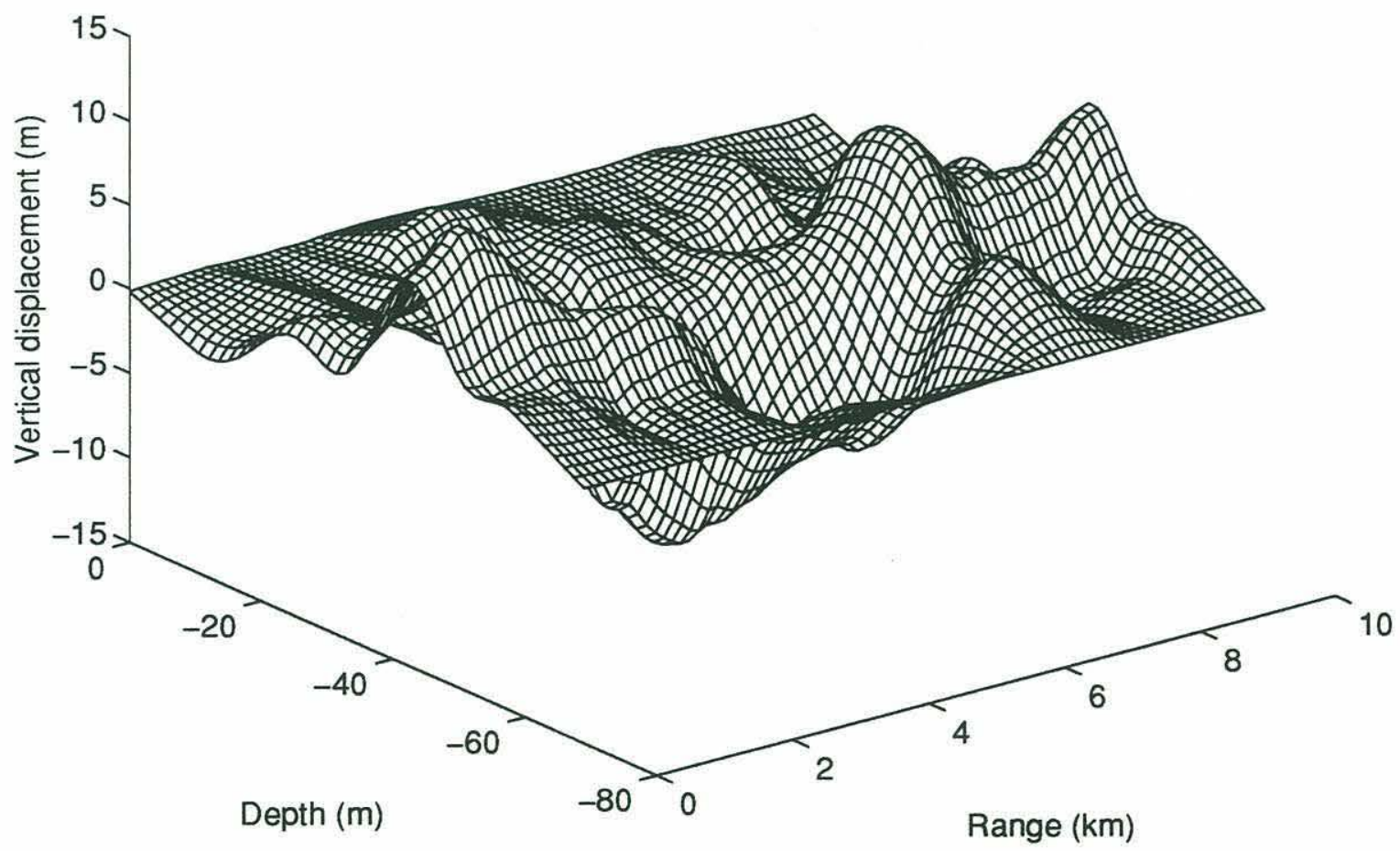

Figure 4.4: One realization of IW vertical displacement from GM spectrum 


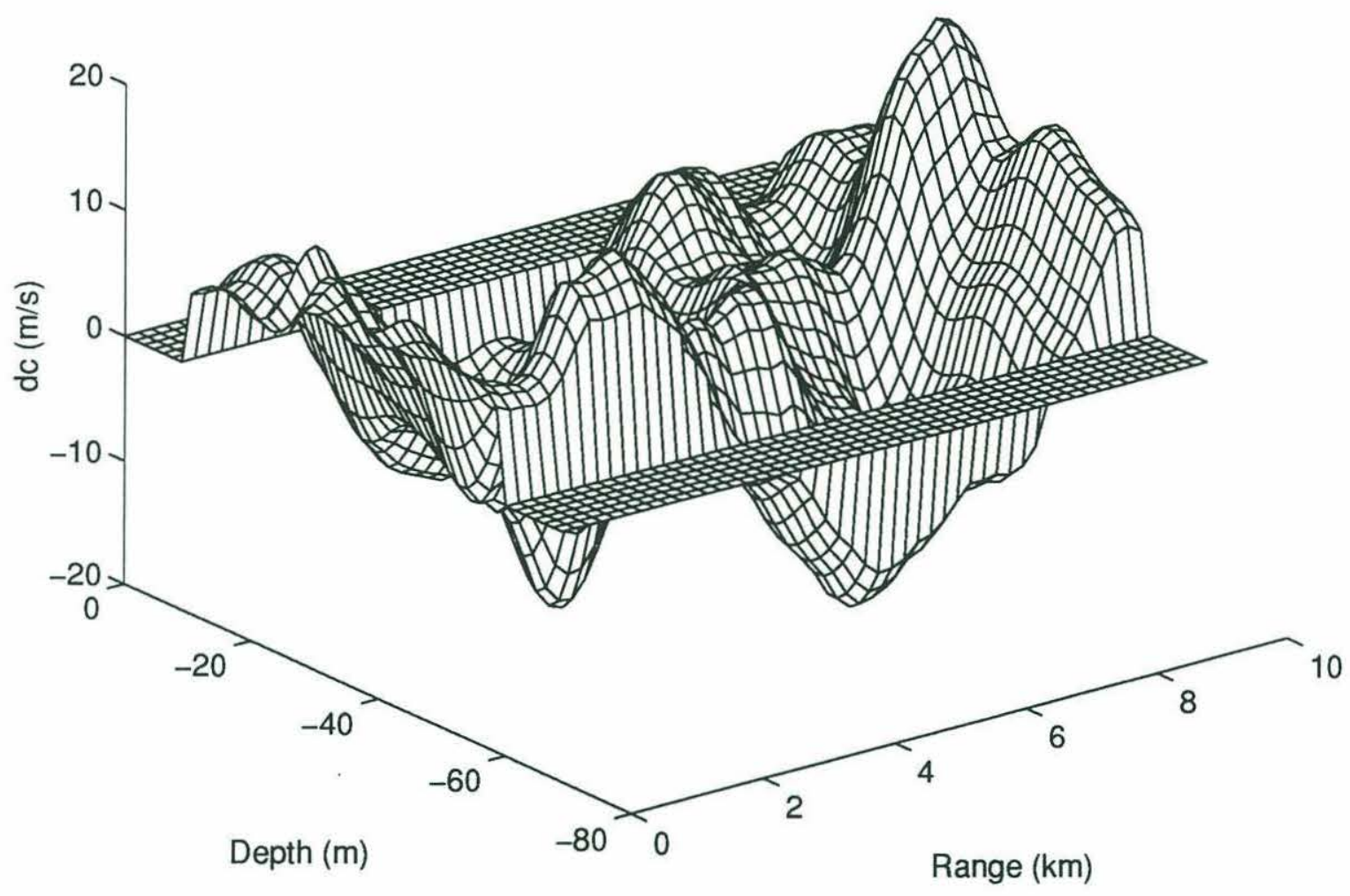

Figure 4.5: One realization of IW induced sound speed fluctuations from GM spectrum

\subsection{Acoustic waveguide model}

To describe the waveguide, we need a water column background profile, the IW fluctuations, and a bottom model. Corresponding to the shallow water three-layer model specified in the last section, the water column background profile is modeled as plotted in Figure 4.6 (a). From the surface to $10 \mathrm{~m}$ depth, the sound speed is a constant of $1530 \mathrm{~m} / \mathrm{s}$; in the middle, from $10-60 \mathrm{~m}$, the sound speed linearly increases with a gradient of $1 \mathrm{~m} / \mathrm{s}$ per meter depth; in the bottom boundary layer, the sound speed is a constant of $1480 \mathrm{~m} / \mathrm{s}$. 
The density in the water column is a constants as $1.0 \mathrm{~g} / \mathrm{cm}^{3}$. As for the sea bottom, we use a simple half-space model with sound speed of $1800 \mathrm{~m} / \mathrm{s}$ and density of $2.0 \mathrm{~g} / \mathrm{cm}^{3}$.

For the above background profile, we use M. Porter's Kraken program (discussed in detail in the next section) to calculate the acoustic normal modes. The acoustic frequency used is $100 \mathrm{~Hz}$. For this frequency, there are 5 trapped modes in the water column. The acoustic mode functions are plotted in Figure 4.6 (c). The mode eigenvalues (i.e. their horizontal wavenumbers) and the interference cycle distances between any two modes are tabulated in Tables 4.1 and 4.2. The source depth is taken to be $30 \mathrm{~m}$. The mode amplitudes for that source depth are shown in Figure 4.6 (b). We see that mode 2 and mode 4 have the largest amplitudes. The cycle distance between mode 2 and mode 4 is $245 \mathrm{~m}$ from Table 4.1. Transmission loss plots in Figure 4.7 show that the dominant cycle distance agrees with modes 2-4 interference.

The above results are for the range-independent model without any fluctuations. Next, we combine internal waves-induced sound speed fluctuations with the background profile and calculate the acoustic transmission characteristics. The sound speed profiles we will use are the sum of the background profile in Figure 4.6(a) and the internal wave induced sound speed fluctuations as plotted in Fig. 4.5. One realization of sound speed profiles is shown in Fig. 4.8. Once we have the sound speed profiles, we can use the Kraken program to evaluate the acoustic field and calculate the acoustic transmission characteristics. 

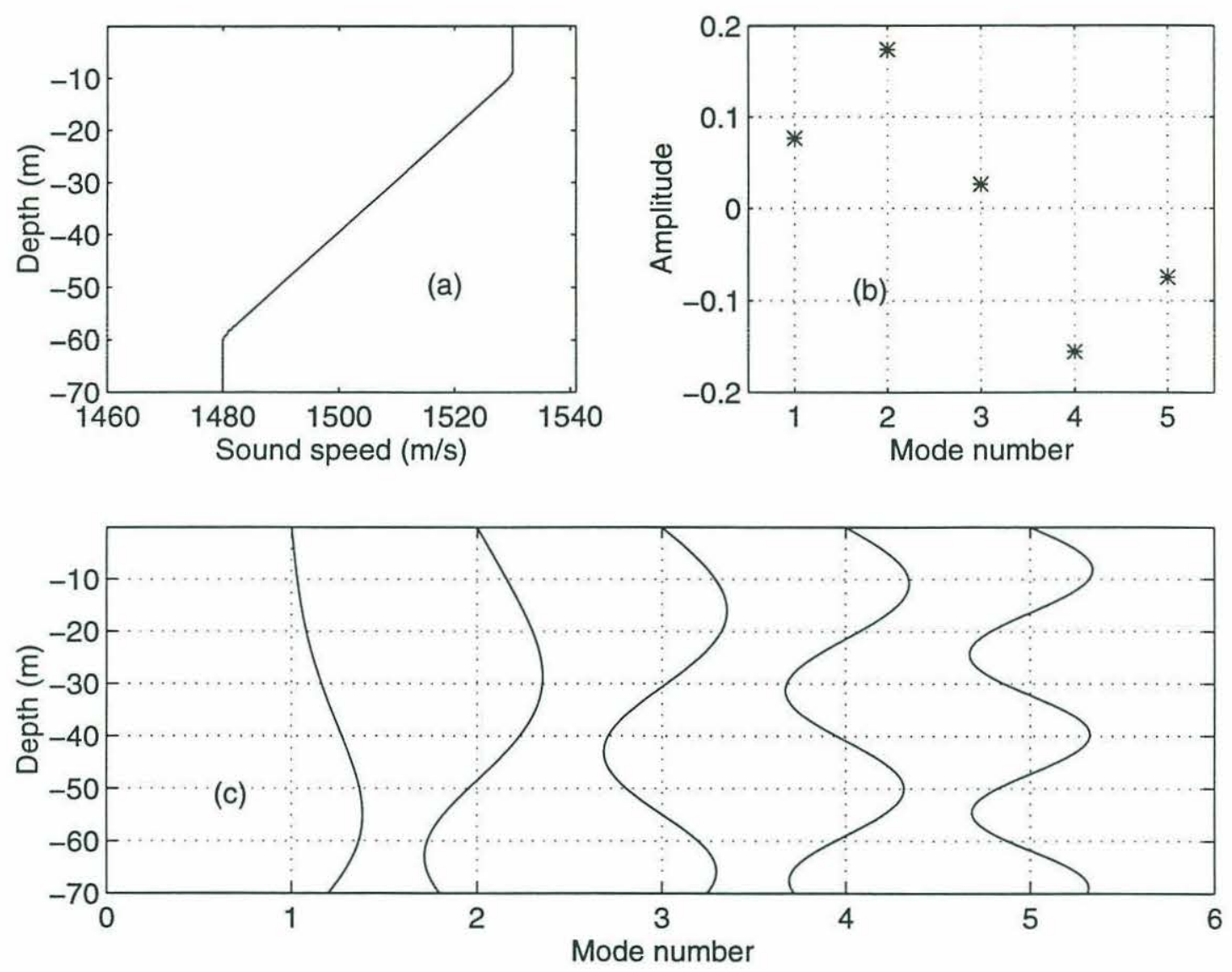

Figure 4.6: (a) Background sound speed profile; (b) mode amplitudes for source depth of $30 \mathrm{~m}$; (c) acoustic modes calculated using Kraken programs. Frequency $=100 \mathrm{~Hz}$. 


\begin{tabular}{|c|c|c|c|c|c|}
\hline Mode \# & 1 & 2 & 3 & 4 & 5 \\
\hline $\begin{array}{l}\text { Horizontal } \\
\text { wavenumber } \\
(\mathrm{rad} / \mathrm{m})\end{array}$ & 0.4186 & 0.4100 & 0.3994 & 0.3842 & 0.3636 \\
\hline $\begin{array}{c}\text { Mode } \\
\text { amplitude }\end{array}$ & 0.0800 & 0.1759 & 0.0137 & 0.1605 & 0.0174 \\
\hline
\end{tabular}

Table 4.1: Horizontal wavenumbers and mode amplitudes for the background sound speed profile with frequency of $100 \mathrm{~Hz}$ and source depth of $30 \mathrm{~m}$.

\begin{tabular}{|c|c|c|c|c|c|}
\hline $\begin{array}{c}\text { Mode } \\
\text { cycle } \\
\text { distance } \\
(\mathrm{m})\end{array}$ & Mode \# 1 & 2 & 3 & 4 & 5 \\
\hline Mode \# 1 & $*$ & 730 & 328 & 183 & 114 \\
\hline 2 & 730 & $*$ & 594 & 244 & 135 \\
\hline 3 & 328 & 594 & $*$ & 413 & 175 \\
\hline 4 & 183 & 183 & 413 & $*$ & 304 \\
\hline 5 & 114 & 135 & 175 & 304 & $*$ \\
\hline
\end{tabular}

Table 4.2: Mode cycle distances between each pair of acoustic modes for the background sound speed profile with frequency of $100 \mathrm{~Hz}$. 


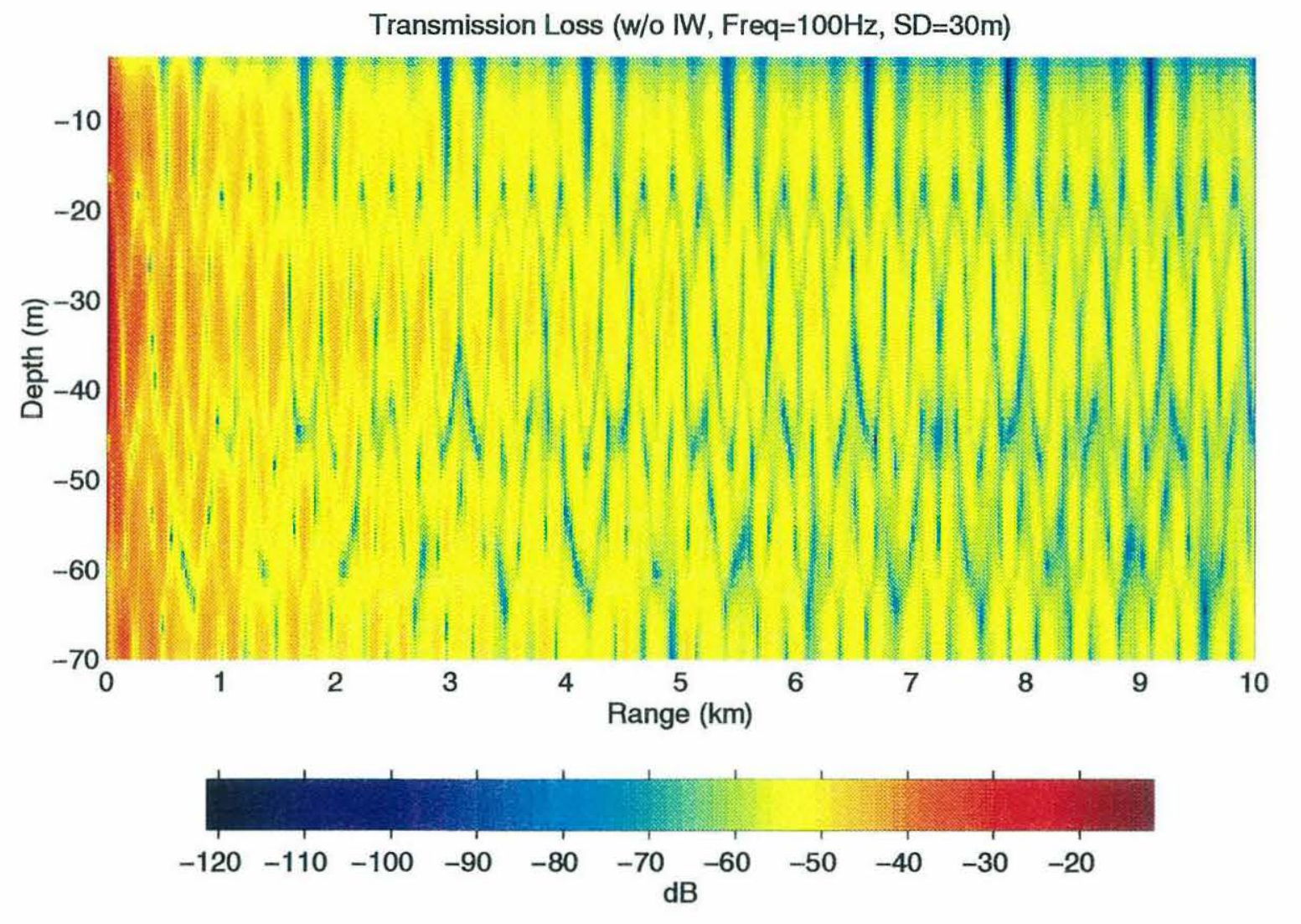

Figure 4.7: 2-D transmission loss for the background sound speed profile model with frequency of $100 \mathrm{~Hz}$ and source depth of $30 \mathrm{~m}$ 


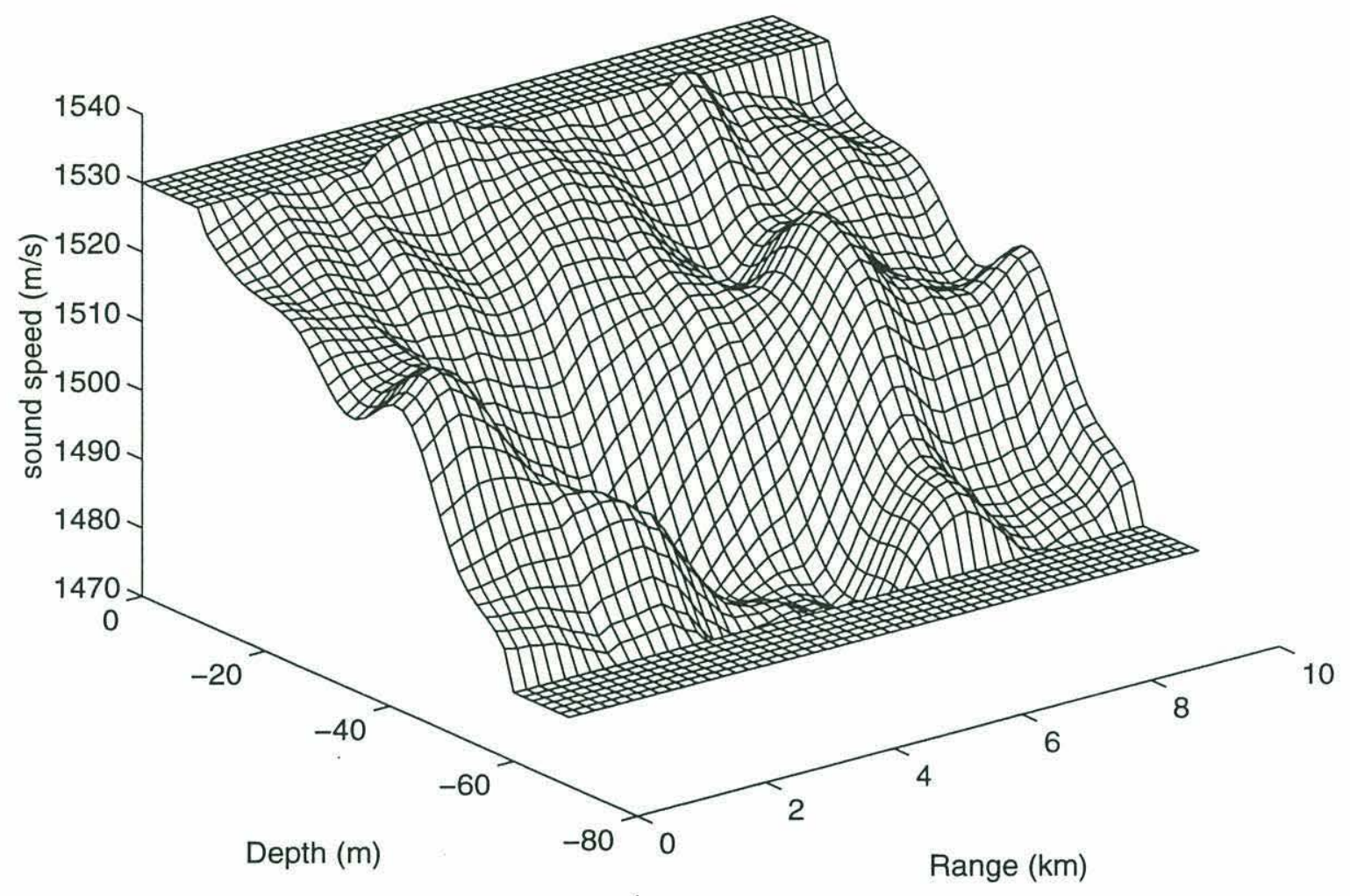

Figure 4.8: One realization of sound speed profiles with IW induced fluctuations 


\subsection{Kraken normal mode program}

The Kraken normal mode program[24] was initially developed by Michael B. Porter in 1980 as part of his Ph.D dissertation. The purpose of his work was to develop a normal mode model which was more robust, accurate and efficient. The work on Kraken was continued after that to add more features and enhance its usability. Now it is a complete software package which can predict transmission loss for range-dependent environments with features such as:

. efficient eigenvalue finding techniques guaranteed to converge

. stable eigenfunction calculation even with multiple ducts

. ability to handle multilayered environments

. inclusion of stratified elastic layers

. inclusion of interfacial roughness

. tabulated surface and bottom reflection coefficients

. choice of perturbational or exact treatment of loss

. calculation of leaky modes

. free, rigid, and homogeneous half-space options for boundary conditions

. adiabatic or coupled mode options for range-dependent problems

. tilted and displaced array calculations

. high-accuracy via extrapolations

. extensions to 3-dimensional varying problems

With all these features, Kraken is an ideal program for the numerical calculations in this thesis. We use Kraken to calculate: 
(1) mode eigenvalues, eigenfunctions, and 2-D (range and depth) pressure field and transmission losses for range-independent environment without internal waves;

(2) mode eigenvalues, eigenfunctions, and 2-D (range and depth) pressure field and transmission losses (both adiabatic and coupled-mode) for range-dependent environments with internal wave caused fluctuations.

With the results calculated using Kraken, we can analyze and evaluate the acoustic scattering by internal waves, compare between adiabatic and coupled-mode methods, and compare between theoretical and numerical results.

Now we will describe briefly the procedure of using Kraken (see documentation by M. B. Porter [24\}for details). The Kraken program is actually part of a complete software package called "Acoustics Toolbox" with structure as shown in Figure 4.9. The structure of Kraken is shown in Figure 4.10. The original development of Kraken had been done on a VAX using VMS Fortran. In order to use it in our UNIX environment at WHOI, the user interface and file formats have been slightly modified by Mr. Arthur Newhall. The output files have also been transferred into MATLAB for further calculations and plotting (see documentation by Arthur Newhall [25] for details).

The input to Kraken is a user-provided environment file called ENVFILin which the user specifies environment parameters such as frequency, sound speed profile, density, bottom properties, etc. The output of Kraken is a file called MODFIL containing calculated eigenvalues (i.e. horizontal wavenumbers) and eigenfunctions (i.e. mode functions). The "field" program then takes MODFIL and a description file (specifying the source depth, receiver depth, range -dependence, etc.) as inputs to calculate the pressure field. There are then various plotting routines available to plot modes, transmission loss, Green's functions, etc. It is usually more convenient and flexible to plot these in MATLAB. 


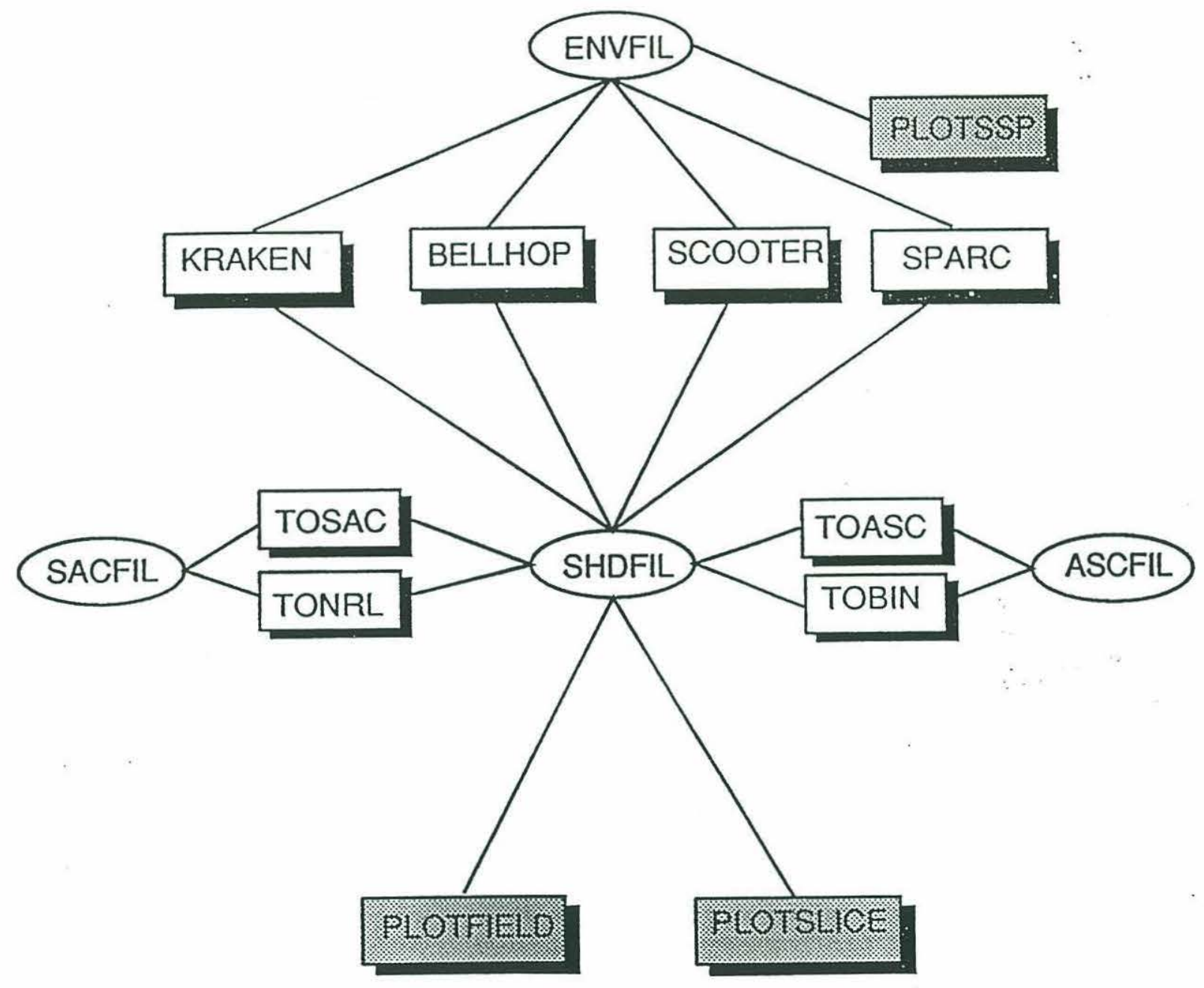

Figure 4.9: Structure of the Acoustics Toolbox 


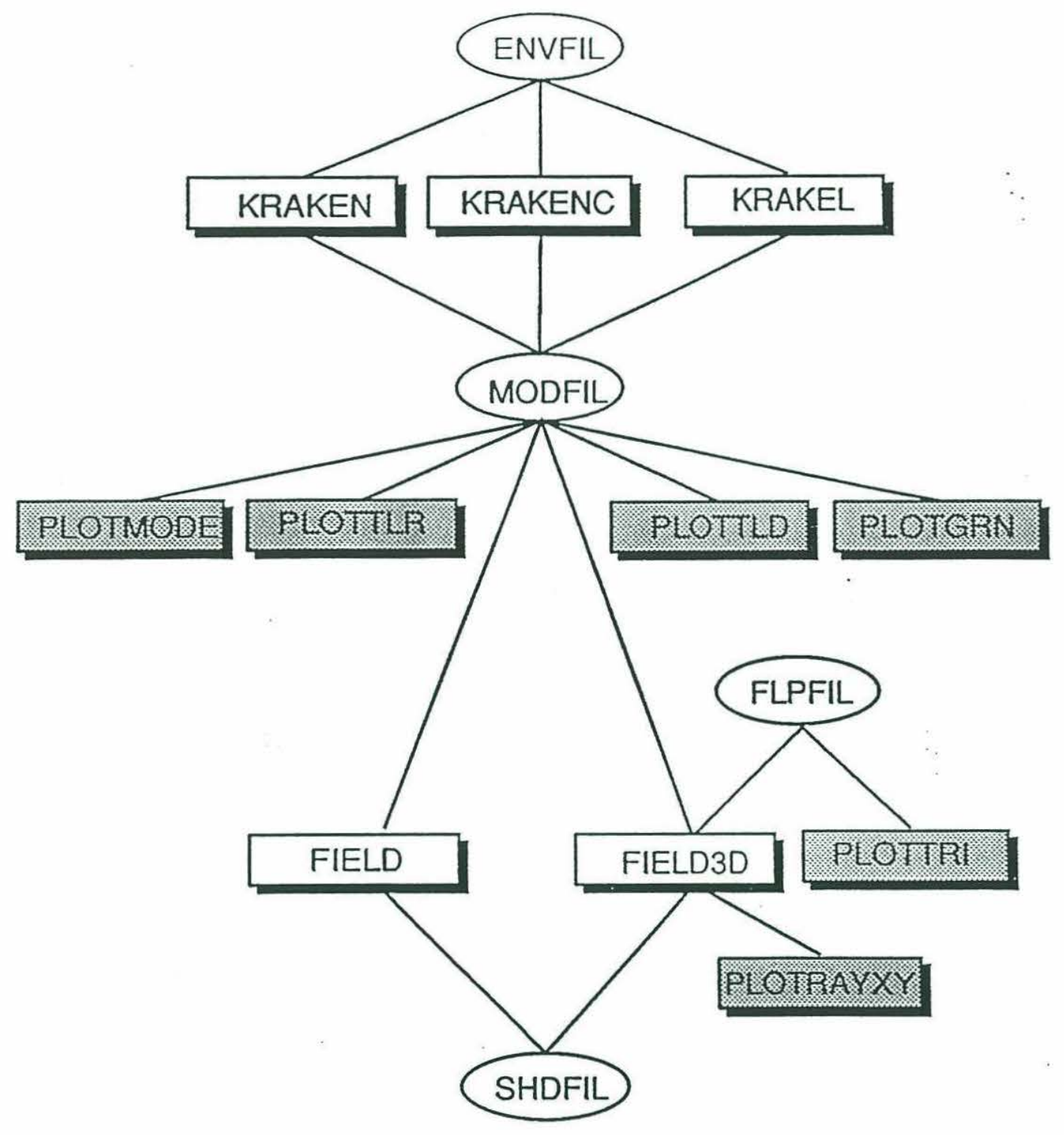

Figure 4.10: Structure of the KRAKEN model 


\subsection{Numerical results and analysis}

In Sections 4.1 and 4.2 we obtained the realizations of sound speed profiles with internal wave induced fluctuations. As we discussed in Section 4.3, we can run the Kraken programs to calculate modes, wavenumbers, pressure field, and transmission loss for such environments. The calculations are divided into two parts which concentrate on 'coherent' and 'incoherent' scattering by IW's. The first part includes:

1) using Kraken to generate the 'coherent' 2-D acoustic intensity and transmission loss for both adiabatic and one-way coupled mode approximations,

2) evaluating the mean and variance of the intensity fluctuations due to internal waves,

3) comparing the results from 'adiabatic' and 'coupled mode' calculations, and

4) evaluating the amplitude fluctuation effects due to modal phase interference.

The second part includes:

1) using modified Kraken programs to generate the 'incoherent' 2-D acoustic intensity fluctuations and transmission losses in the adiabatic approximation,

2) evaluating the mean and variance of the intensity fluctuations,

3) calculating the mean and variance of incoherent adiabatic intensity fluctuation using the theoretical results developed in Chapter 3, and

4) comparing results from the theory developed in Chapter 3 and Kraken calculations.

\subsubsection{Coherent intensity calculations}

We want to compare between adiabatic and coupled mode methods and see how large the difference is. We also wish to discuss the dependence of the coherent scattering on range, frequency, and IW amplitude. To do these, calculations are performed as follows. For each realization, we 
(1) run 'kraken' to generate the acoustic modes and wavenumbers for sound speed profiles at different ranges,

(2) run 'field' with the 'adiabatic' option to generate the 2-D acoustic pressure field in the adiabatic approximation,

(3) run 'field' with the 'coupled' option to generate the 2-D acoustic pressure field for the coupled mode method, and

(4) calculate the intensity field and transmission losses.

The number of realizations is chosen according to the convergency of the mean and variance. When these statistics converge to a fixed level, we say the number of realizations is large enough. Figure 4.11 shows the mean and variance vs. number of realizations.
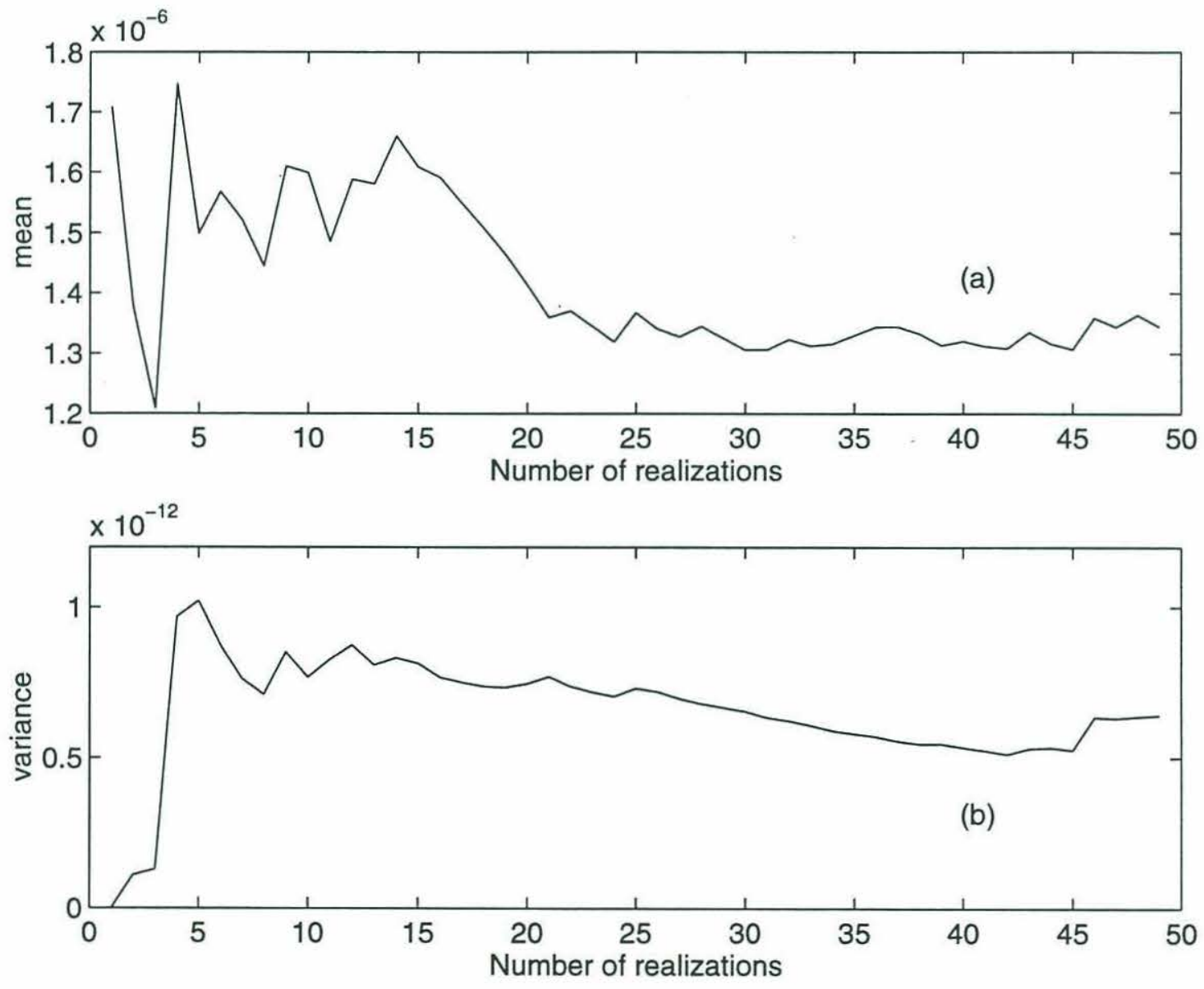

Figure 4.11: Mean and variance of intensity vs. number of realizations at range of $5 \mathrm{~km}$ and receiver depth of $30 \mathrm{~m}$. Frequency $=100 \mathrm{~Hz}$. 
Thus, after 49 realizations, we

(5) calculate the mean and variance of acoustic intensity.

Then we change the acoustic frequency and repeat (1) - (5). The frequencies used are 50, 100, and $200 \mathrm{~Hz}$. Then we repeat (1) - (5) for different IW field amplitudes. The amplitudes used are 10,20, and 30 meter (IW amplitude in this thesis refers to half of the peakto-peak value).

We now present and discuss the results. The following results are from the calculations with frequency $=100 \mathrm{~Hz}$, IW amplitude $=10 \mathrm{~m}$, and source depth of $30 \mathrm{~m}$, unless otherwise stated.

We first look at intensity and transmission loss(TL) to examine means, variances, and the difference between adiabatic and coupled mode calculations. Fig. 4.12 shows adiabatic transmission loss curves at a depth of $30 \mathrm{~m}$. We can see that the TL curve for the average value of intensities for 49 realizations approaches the TL without IW i.e. the mean of the intensity fluctuations due to IW is zero. Looking at the green TL curves for 49 realizations in Fig 4.12, we notice a very distinct characteristic of the horizontal structure of the variance of the TL, i.e. that the variance is small at the beginning, increases with range up to about the mid-point of the $10 \mathrm{~km}$ range, and then decreases with range. Fig. 4.13 shows the standard deviation of the adiabatic intensity fluctuations normalized by the mean value of the intensity. We again notice the "increasing/decreasing" horizontal structure of the variance. In the latter part of Section 3.3, we predicted that the variance of coherent intensity fluctuation first increases with range up to halfway of the dominant internal wave cycle and then decreases with range and goes back to about the same level as that at source at the end of the internal wave cycle. From Fig. 4.3, the horizontal wavenumber spectrum of the simulated internal waves, we can see that the most energy is contained in the longest wavelengths, which are around $10 \mathrm{~km}$ in Fig 4.3. We thus see that the Kraken results agree 
with the prediction in Sec.3.3 very well. Also in Sec.3.3, we knew that this range dependence of coherent intensity variance is related to the modal phase fluctuations. We thus want to examine the Kraken results for the modal phase fluctuations.

Fig. 4.17 shows the horizontal wavenumber perturbations due to IW vs. range (for one realization). Fig. 4.18 is the running integral of the wavenumber perturbations, i.e. the mode phase fluctuations. According to the theoretical work in Section 3.3, the phase fluctuation starts from zero at the beginning and goes back to zero at the end of the internal wave cycle. From Fig. 4.18, we see that the phase fluctuation is indeed back to near zero at the end of the dominant internal wave cycle, which is $10 \mathrm{~km}$ according to Fig.4.3.

Fig. 4.14 is the same plot as Fig.4.13 but for a fully coupled mode calculation. We notice that it is very similar to the adiabatic result in Fig.4.13. This can be explained by the fact that the mode coupling effect is not important in this case. Fig. 4.15 shows the TL difference between "coupled mode" and "adiabatic" results. We notice that the difference increases with range. This agrees qualitatively with Eq.(3.128), in which the mode coupling effect is proportional to range. However, the difference is basically very small. From Figs 4.16 (a) and (b), which are the one-slice plots of the TL difference at depth of $10 \mathrm{~m}$ and the moving average of the TL difference over range, we find that the TL difference is only about $0.15 \mathrm{~dB} / \mathrm{km}$. From Fig. 4.15 , the TL difference is the biggest at depth of $10 \mathrm{~m}$. Thus we can say that $0.15 \mathrm{~dB} / \mathrm{km}$ is the upper limit in this case. The peaks in the TL difference are indications of the shifting of nulls in the mode interference pattern, i.e. the difference in wavenumbers between adiabatic and coupled mode. The vertical structure of the difference, compared with 2-D TL plot in Figure 4.7, reflects the basic mode structure.

The small TL difference between adiabatic and coupled mode shows that the mode coupling effect is weak for our range of $10 \mathrm{~km}$ and frequency of $100 \mathrm{~Hz}$ in the simulated internal wave field, and then the adiabatic approximation is accurate enough. This con- 
forms to our predictions for the acoustic scattering by linear internal waves in Chapter 3 . Specifically, according to Table 4.2 , the longest mode cycle distance is $730 \mathrm{~m}$, which is the cycle distance between mode 1 and mode 2 . This cycle distance corresponds to the horizontal wavenumber of $1.371 / \mathrm{km}$. The dominant mode cycle distance in this case, which is between mode 2 and mode 4 , is $244 \mathrm{~m}$ according to Tables 4.1 and 4.2 . The corresponding horizontal wavenumber is $4.11 / \mathrm{km}$. From Fig.4.3, we find that the IW spectrum magnitudes at wavenumbers $1.371 / \mathrm{km}$ and $4.11 / \mathrm{km}$ are about $20 \mathrm{~dB}$ and $27 \mathrm{~dB}$ lower than the biggest value respectively. Thus according to our prediction, the mode coupling should be very weak and the adiabatic approximation is good for this case. This agrees with the numerical results.

Next we look at IW amplitudes of $10 \mathrm{~m}, 20 \mathrm{~m}$, and $30 \mathrm{~m}$ and repeat the above simulation and calculation process. What we are interested in are the changes- of TL difference between adiabatic and coupled mode with IW amplitude and the change of the standard deviation of acoustic intensity with IW amplitude. The 2-D TL difference (between adiabatic and coupled mode) results for IW amplitudes $20 \mathrm{~m}$ and $30 \mathrm{~m}$ are shown in Figs. 4.19-4.21. Compared with the results for IW amplitude of $10 \mathrm{~m}$ in Figures 4.15-4.16, we find that the TL difference between adiabatic and coupled mode calculations barely changes with the IW amplitude. This is a very interesting result. The TL difference actually reflects the ratio of adiabatic intensity and coupled mode intensity, not the absolute intensity difference between adiabatic and coupled mode calculations. The adiabatic intensity is related to the IW energy most of which is contained in the longest wavelengths. And mode coupling is proportional to the IW energy at the wavenumbers corresponding to the mode cycle distances. Thus the TL difference between adiabatic and coupled mode depends on the ratio of IW energy at the longest wavelengths to IW energy at the wavenumbers corresponding to the acoustic mode cycle distances. In our calcula- 
tions, we only change the IW amplitude, not the IW spectrum shape and hence not the ratio of IW energy spectrum magnitudes at different wavenumbers. Thus the TL difference between adiabatic and coupled mode basically doesn't change with IW amplitude.

Figure 4.22 shows the standard deviations for different IW amplitudes, we can see that the deviation increases with IW amplitudes in nearly linear fashion. This agrees well with the theory in Chapter 3.

We next look at the TL difference between adiabatic and coupled mode calculations and the standard deviation of intensity for different frequencies. Similarly, we repeat the calculations for different acoustic frequencies $50 \mathrm{~Hz}, 100 \mathrm{~Hz}$, and $200 \mathrm{~Hz}$. The results are shown in Figs. 4.23 - 4.25. From Figs 4.23 - 4.25, we can see that the difference between adiabatic and coupled mode calculations increases with frequency. According to the above discussions, the TL difference is related to the ratio of IW energy at the longest wavelengths to IW energy at the wavenumbers corresponding to the acoustic mode cycle distances. According to Section 3.4, the mode cycle distance increases with frequency. And from the wavenumber spectrum in Fig. 4.3, the larger the mode cycle distance, the higher the IW spectrum magnitude at the corresponding wavenumber, and hence the smaller the ratio of IW energy at the longest wavelengths to IW energy at wavenumbers corresponding to the acoustic mode cycle distances. Thus the TL difference between adiabatic and coupled mode calculations increases with frequency. We can evaluate this frequency dependence more quantitatively by calculating mode cycle distances and examining mode excitation at source for the frequencies of $50 \mathrm{~Hz}, 100 \mathrm{~Hz}$, and $200 \mathrm{~Hz}$. Tables $4.3-4.6$ shows the horizontal wavenumber and mode amplitude for each mode and mode cycle distance between every pair of acoustic modes for the two different frequencies of $50 \mathrm{~Hz}$ and $200 \mathrm{~Hz}$. From Tables 4.3 and 4.4, which shows the results for the case of $50 \mathrm{~Hz}$, we find that the dominant mode cycle distance is between mode 1 and mode 2 and is $542 \mathrm{~m}$. 
From the $200 \mathrm{~Hz}$ results in Tables 4.5 and 4.6 , we find that the dominant mode cycle distance is between mode 2 and mode 3 and is equal to $767 \mathrm{~m}$. The $100 \mathrm{~Hz}$ results are a little more complicated, which we already saw in Tables 4.1 and 4.2 . For $100 \mathrm{~Hz}$, the dominant mode cycle distance is between mode 2 and mode 4 , which is equal to $244 \mathrm{~m}$. However, the longest mode cycle distance is $730 \mathrm{~m}$ which is between mode 1 and mode 2 . Examining the modal amplitudes and doing some simple calculations, we find that mode 1-2 interference strength is only about $1.8 \mathrm{~dB}$ less than the mode $2-4$ interference (by calculating the ratio of energy contained in mode 1 and mode 2 to energy contained in mode 2 and mode 4). From the IW wavenumber spectrum in Fig. 4.3, we can decide that the mode 1-2 cycle distance is the most important in determining mode coupling strength. Based on the above results, we can decide that the maximum mode coupling points in the IW wavenumber spectrum are $1 / 541 \mathrm{~m}=1.81 / \mathrm{km}, 1 / 730 \mathrm{~m}=1.41 / \mathrm{km}$, and $1 / 767 \mathrm{~m}=1.31 / \mathrm{km}$ for acoustic frequencies of $50 \mathrm{~Hz}, 100 \mathrm{~Hz}$, and $200 \mathrm{~Hz}$. From the IW wavenumber spectrum in Fig. 4.3, we find that the spectrum magnitudes at maximum coupling points for $100 \mathrm{~Hz}$ and $200 \mathrm{~Hz}$ are $1.5 \mathrm{~dB}$ and $3.5 \mathrm{~dB}$ higher than the spectrum magnitude at the maximum coupling point for $50 \mathrm{~Hz}$, respectively. Thus the TL difference between adiabatic and coupled mode calculations is the biggest for $200 \mathrm{~Hz}$ and the smallest for $50 \mathrm{~Hz}$. This agrees with the Kraken calculation results shown in Fig.4.25.

Fig. 4.26 shows the average ratio of standard deviation to mean value of the acoustic intensity for frequencies of $50 \mathrm{~Hz}, 70.7 \mathrm{~Hz}, 100 \mathrm{~Hz}, 141 \mathrm{~Hz}$, and $200 \mathrm{~Hz}$ at depths of $10 \mathrm{~m}$, $30 \mathrm{~m}$, and $50 \mathrm{~m}$. We can see that the ratio increases with frequency. The explanation for this is related to our discussion about the mode phase fluctuations in Chapter 3. According to Eq. (3.46), the horizontal wavenumber perturbation increases quadratically with frequency. So the modal phase perturbation, which is the integral of the wavenumber perturbation over range, should also increase quadratically with frequency. The variance of the 
modal phase fluctuations then also increases with frequency. So does the variance of coherent intensity fluctuation according to our discussion in the latter part of Sec.3.3. In Figure 4.26, we also notice a linear dependence between the ratio of STD/MEAN and the base 10 logarithm of frequency. Thus we want to do linear lease square fitting according to the following formula:

$$
\frac{\mathrm{STD}}{\mathrm{MEAN}}=a \log _{10}(f)+b
$$

After calculation, we obtain that $a \approx 0.55$ and $b$ is dependent on the receiver depth.

\begin{tabular}{|c|c|c|c|}
\hline Mode \# & 1 & 2 & 3 \\
\hline $\begin{array}{l}\text { Horizontal } \\
\text { wavenumber } \\
(\mathrm{rad} / \mathrm{m})\end{array}$ & 0.2068 & 0.1952 & 0.1754 \\
\hline $\begin{array}{c}\text { Mode } \\
\text { amplitude }\end{array}$ & 0.1148 & 0.1483 & 0.0149 \\
\hline
\end{tabular}

Table 4.3: Horizontal wavenumbers and mode amplitudes for the background sound speed profile with frequency of $50 \mathrm{~Hz}$ and source depth of $30 \mathrm{~m}$.

\begin{tabular}{|c|c|c|c|}
\hline $\begin{array}{c}\text { Mode } \\
\text { cycle } \\
\text { distance }(\mathrm{m})\end{array}$ & Mode \# 1 & 2 & 3 \\
\hline Mode \# 1 & $*$ & 542 & 200 \\
\hline 2 & 542 & $*$ & 317 \\
\hline 3 & 200 & 317 & $*$ \\
\hline
\end{tabular}

Table 4.4: Mode cycle distances between each pair of acoustic modes for the background sound speed profile with frequency of $50 \mathrm{~Hz}$. 


\begin{tabular}{|c|c|c|}
\hline Mode \# & $\begin{array}{c}\text { Horizontal } \\
\text { wavenumber } \\
(\mathrm{rad} / \mathrm{m})\end{array}$ & $\begin{array}{c}\text { Mode } \\
\text { amplitude }\end{array}$ \\
\hline 1 & 0.8427 & 0.0209 \\
\hline 2 & 0.8329 & 0.1296 \\
\hline 3 & 0.8247 & 0.1838 \\
\hline 4 & 0.8167 & 0.0446 \\
\hline 5 & 0.8070 & 0.1553 \\
\hline 6 & 0.7947 & 0.0525 \\
\hline 7 & 0.7797 & 0.1634 \\
\hline 8 & 0.7617 & 0.0105 \\
\hline 9 & 0.7407 & 0.1657 \\
\hline 10 & 0.7165 & 0.0533 \\
\hline
\end{tabular}

Table 4.5: Horizontal wavenumbers and mode amplitudes for the background sound speed profile with frequency of $200 \mathrm{~Hz}$ and source depth of $30 \mathrm{~m}$. 


\begin{tabular}{|c|l|l|l|l|l|l|c|c|c|c|}
\hline $\begin{array}{c}\text { Mode } \\
\text { cycle } \\
\text { distance } \\
(\mathrm{m})\end{array}$ & $\begin{array}{c}\text { Mode } \\
\# 1\end{array}$ & 2 & 3 & 4 & 5 & 6 & 7 & 8 & 9 & 10 \\
\hline Mode \# 1 & $*$ & 643 & 350 & 242 & 176 & 131 & 100 & 78 & 62 & 50 \\
\hline 2 & & $*$ & 767 & 387 & 242 & 164 & 118 & 88 & 68 & 54 \\
\hline 3 & & & $*$ & 781 & 354 & 209 & 139 & 99 & 75 & 58 \\
\hline 4 & & & & $*$ & 648 & 286 & 170 & 114 & 83 & 63 \\
\hline 5 & & & & & $*$ & 511 & 230 & 139 & 95 & 69 \\
\hline 6 & & & & & & $*$ & 419 & 190 & 116 & 80 \\
\hline 7 & & & & & & & $*$ & 349 & 161 & 99 \\
\hline 8 & & & & & & & & $*$ & 299 & 139 \\
\hline 9 & & & & & & & & & $*$ & 260 \\
\hline 10 & & & & & & & & & & $*$ \\
\hline
\end{tabular}

Table 4.6: Mode cycle distances between each pair of acoustic modes for the background sound speed profile with frequency of $200 \mathrm{~Hz}$. 


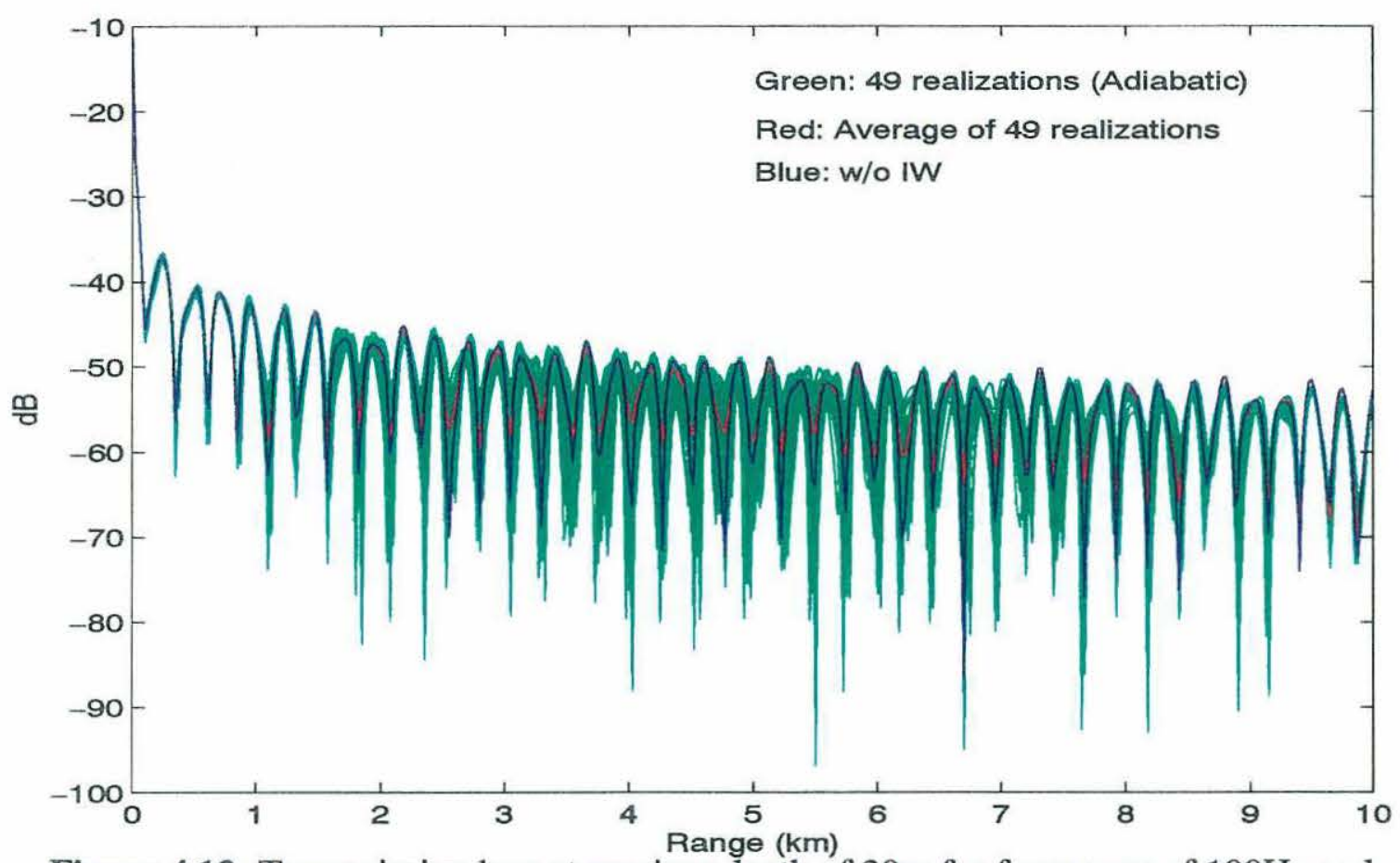

Figure 4.12: Transmission loss at receiver depth of $30 \mathrm{~m}$ for frequency of $100 \mathrm{~Hz}$ and source depth of $30 \mathrm{~m}$.

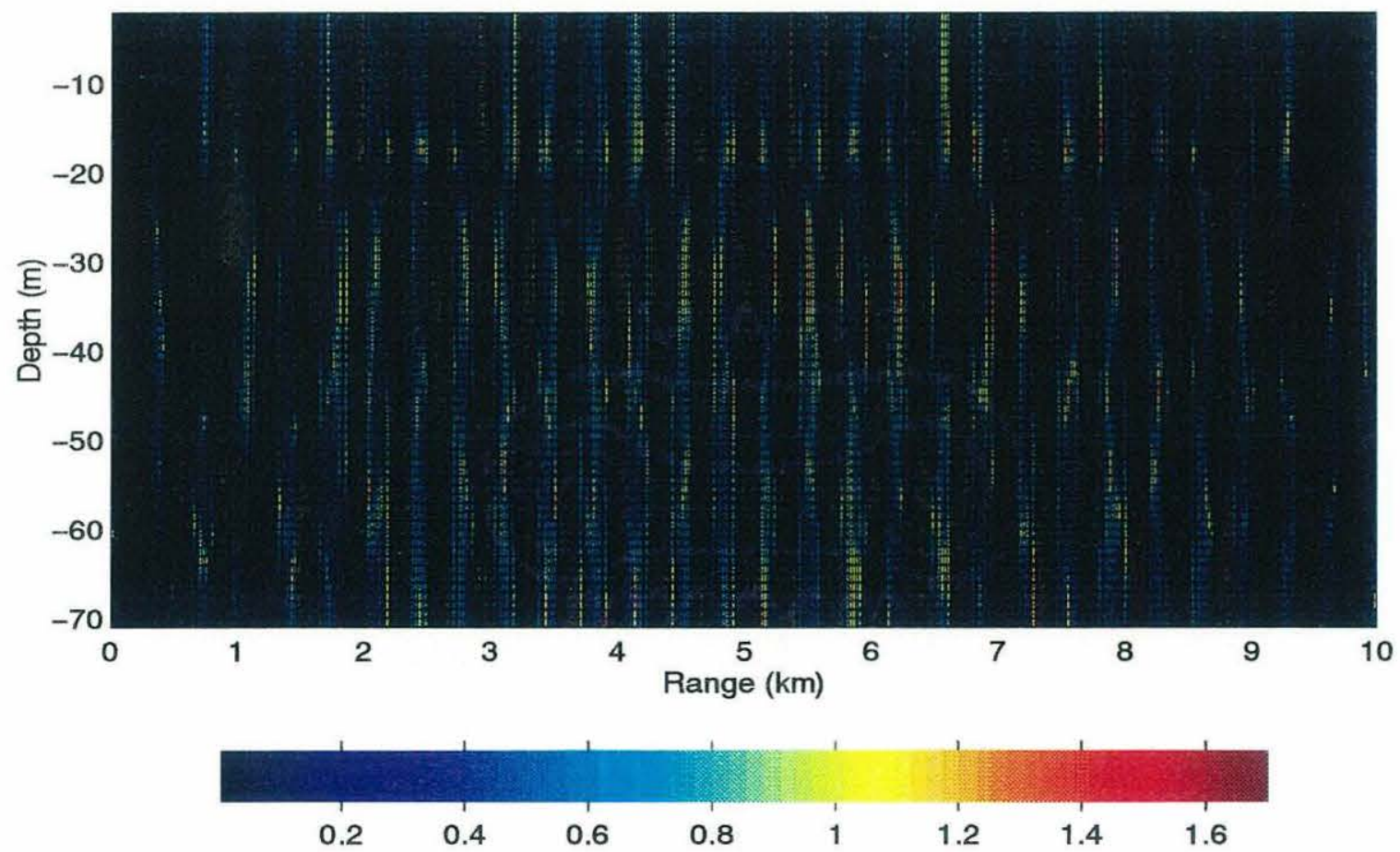

Figure 4.13: Standard deviation normalized by mean value for the acoustic intensity from adiabatic calculation. IW amplitude $=10 \mathrm{~m}$ and frequency $=100 \mathrm{~Hz}$ 

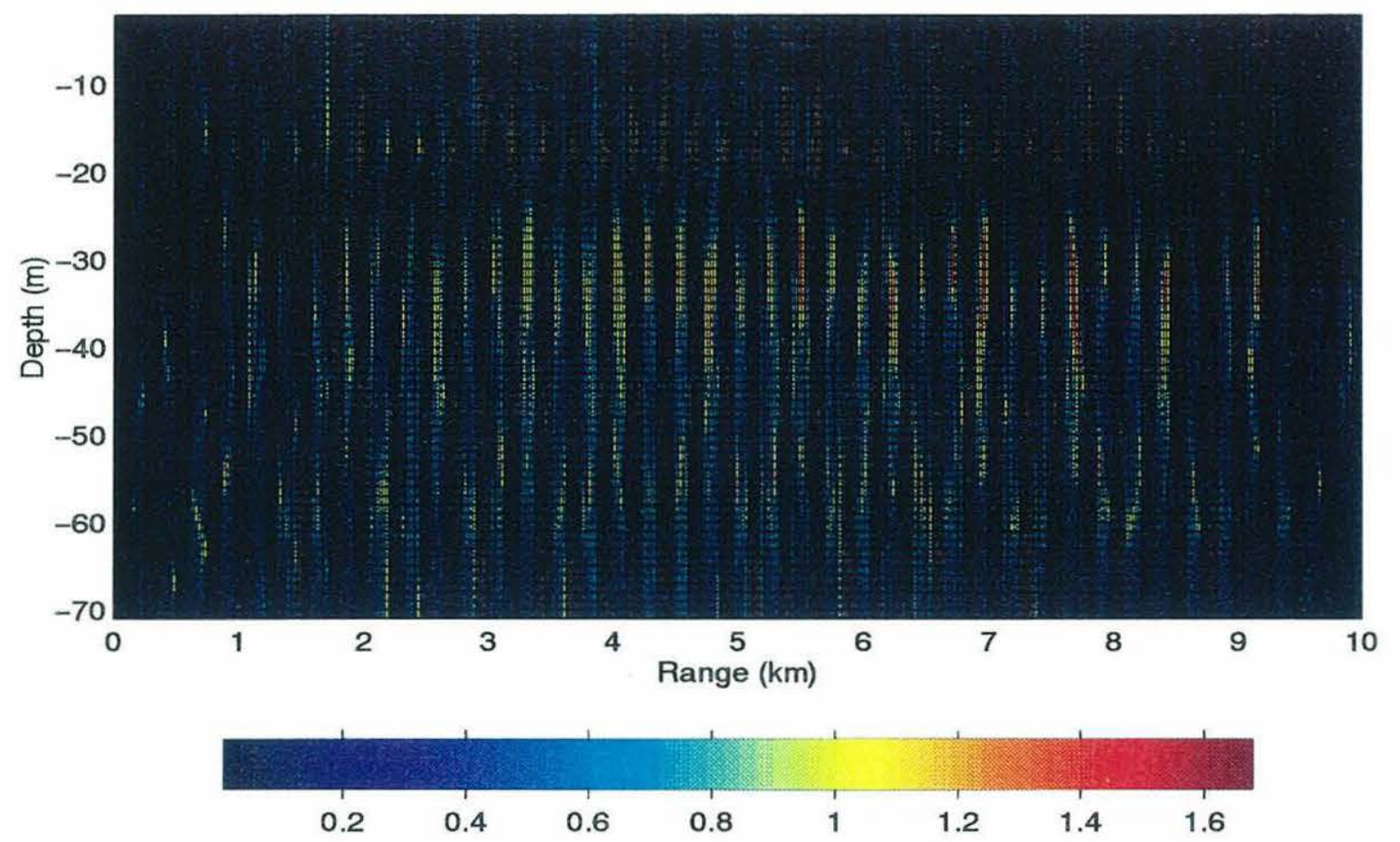

Figure 4.14: Standard deviation normalized by mean value for the acoustic intensity from coupled mode calculation. IW amplitude $=10 \mathrm{~m}$ and acoustic frequency of $100 \mathrm{~Hz}$

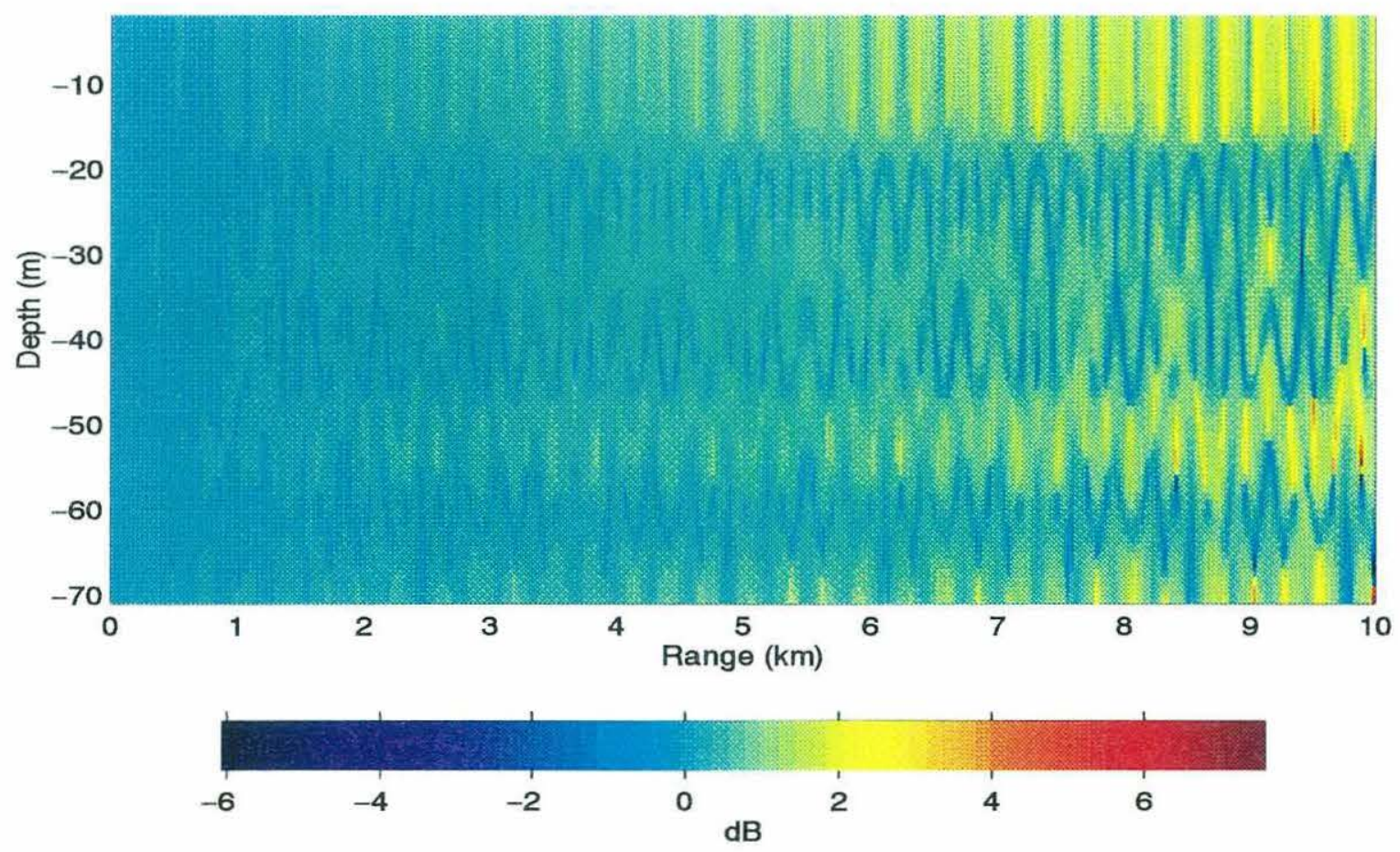

Figure 4.15: Transmission loss difference between adiabatic approximation and coupled mode calculations (average of 49 realizations). Frequency $=100 \mathrm{~Hz}$. 


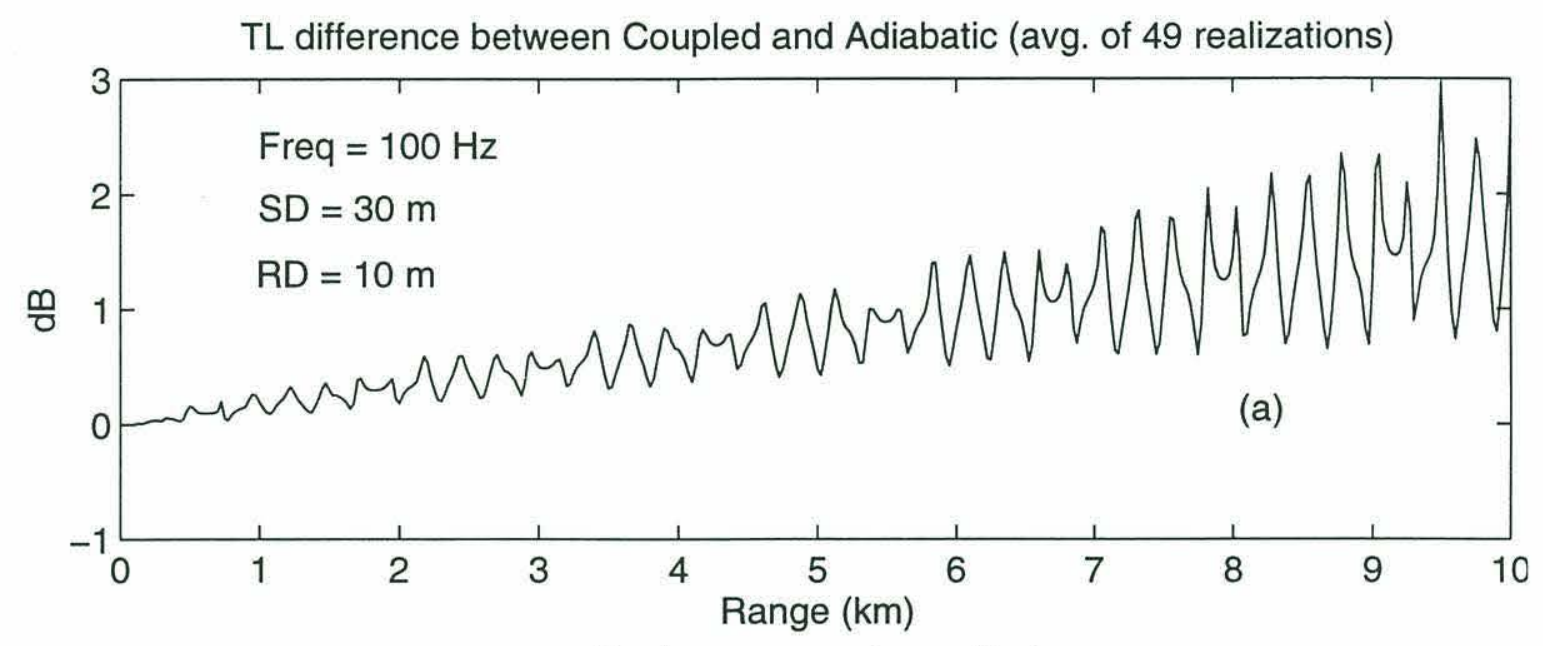

Moving average (over $1 \mathrm{~km}$ )

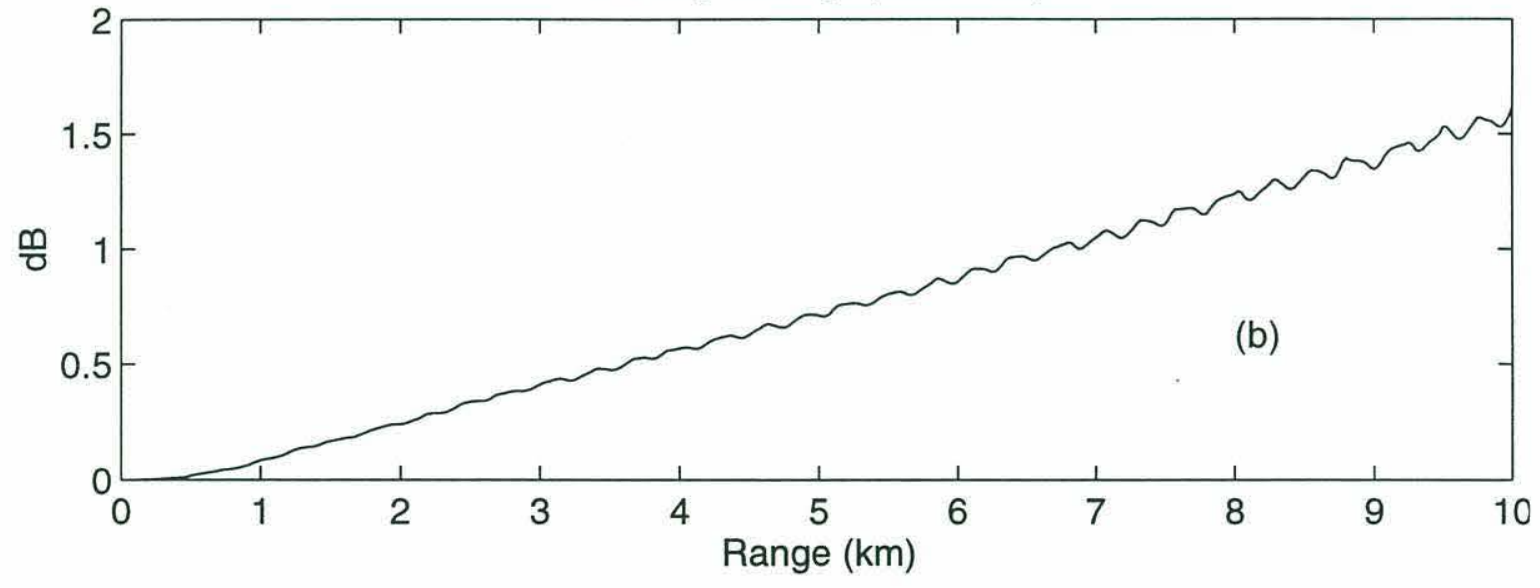

Figure 4.16: Transmission loss difference between adiabatic approximation and coupled mode calculations. Frequency $=100 \mathrm{~Hz}$, source depth $=30 \mathrm{~m}$, and receiver depth $=10 \mathrm{~m}$. 

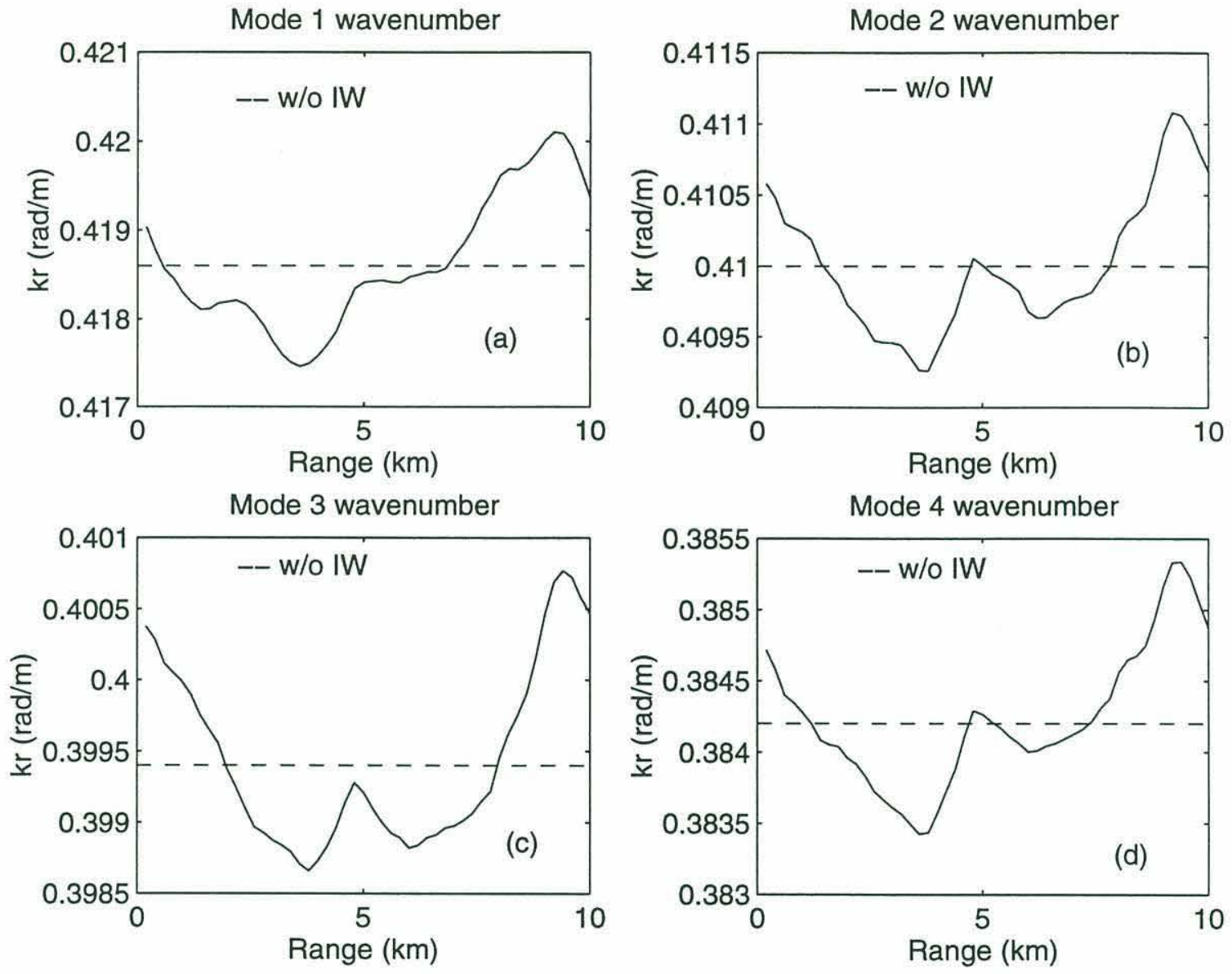

Figure 4.17: Acoustic horizontal wavenumber perturbations vs. range for one realization of IW induced sound speed fluctuations. Frequency $=100 \mathrm{~Hz}$. 

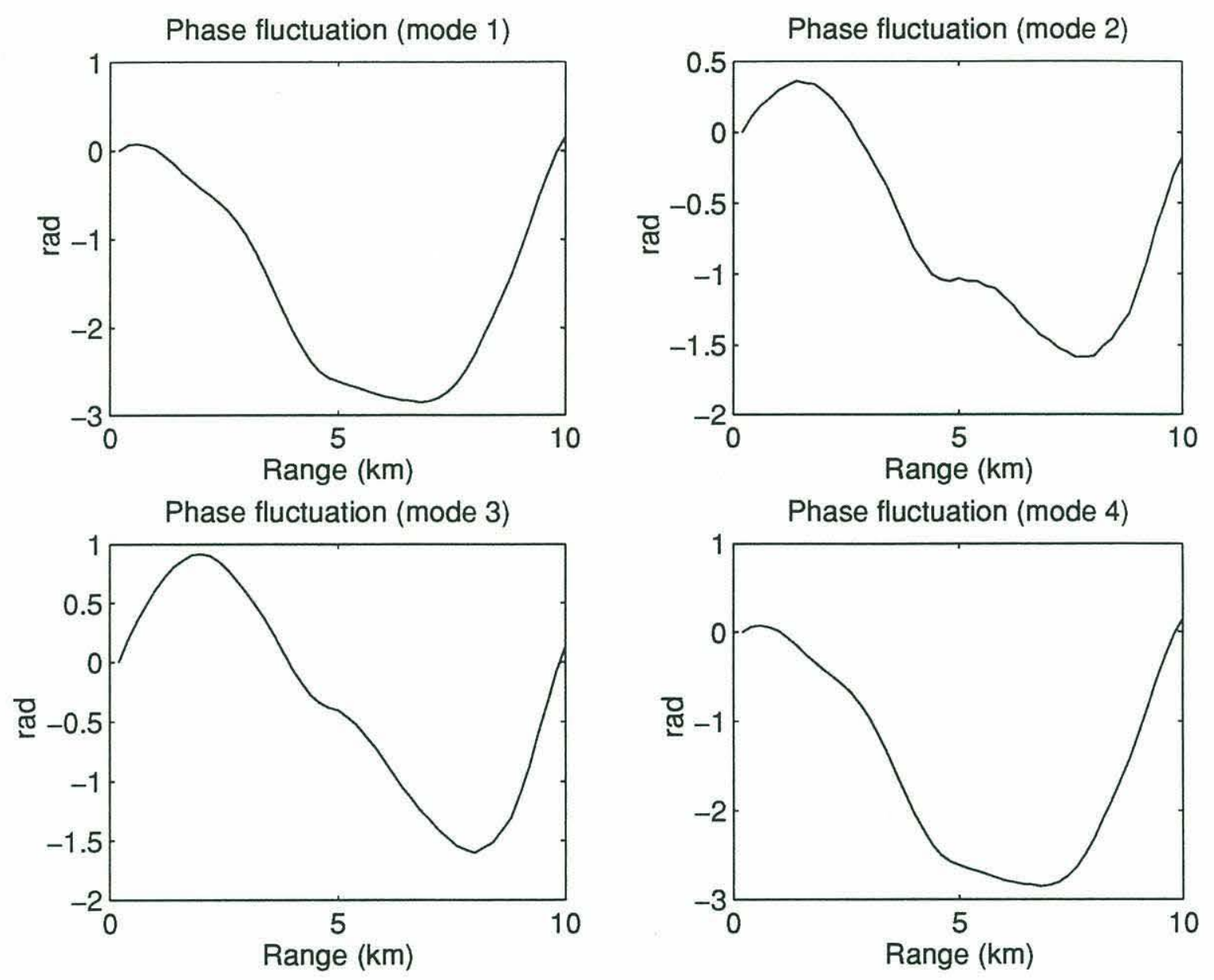

Figure 4.18: Modal phase perturbations vs. range due to the horizontal wavenumber perturbations shown in Fig. 4.17. 


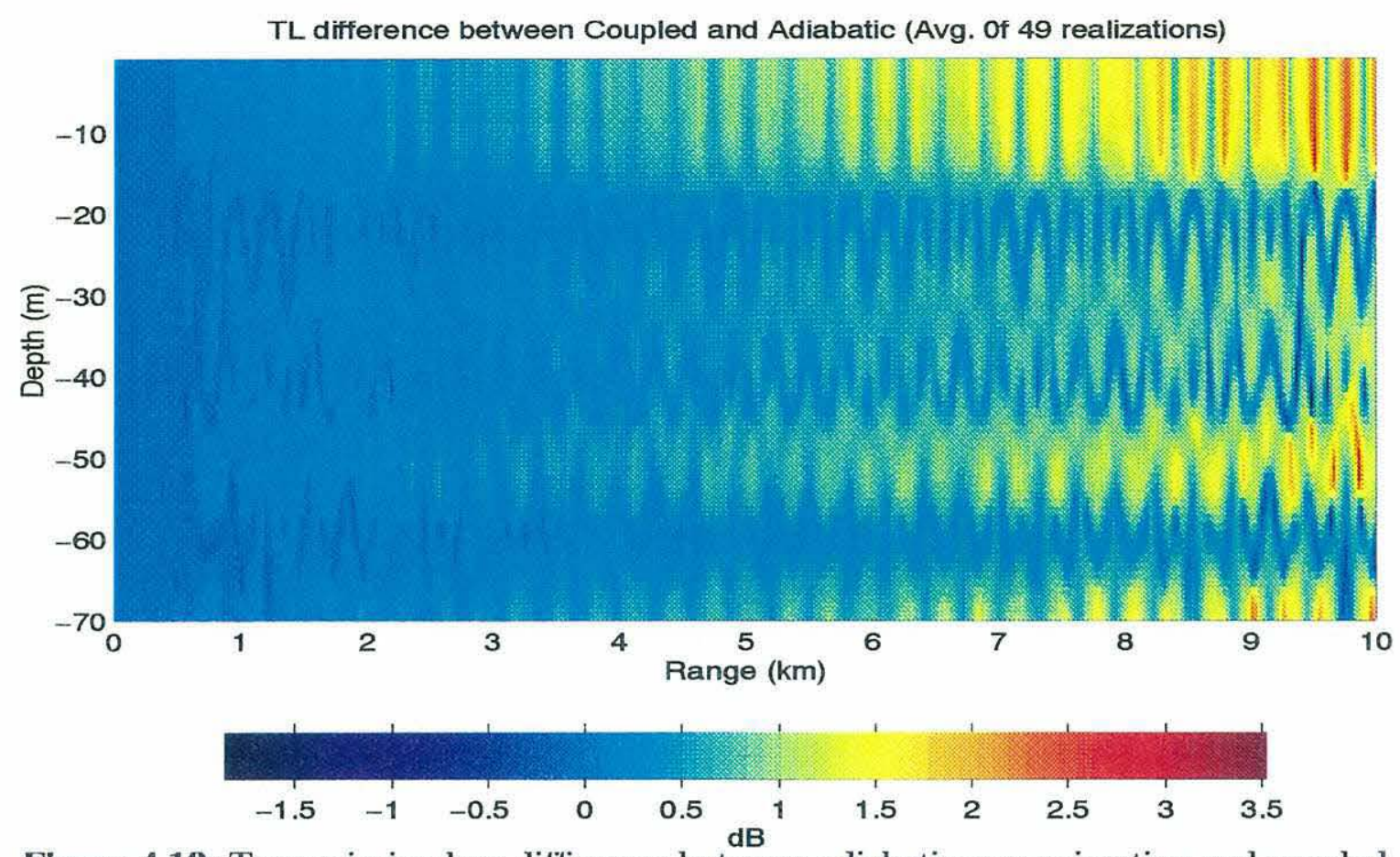

Figure 4.19: Transmission loss difference between adiabatic approximation and coupled mode calculations (average of 49 realizations) for frequency of $100 \mathrm{~Hz}$ and IW amplitude of $20 \mathrm{~m}$.

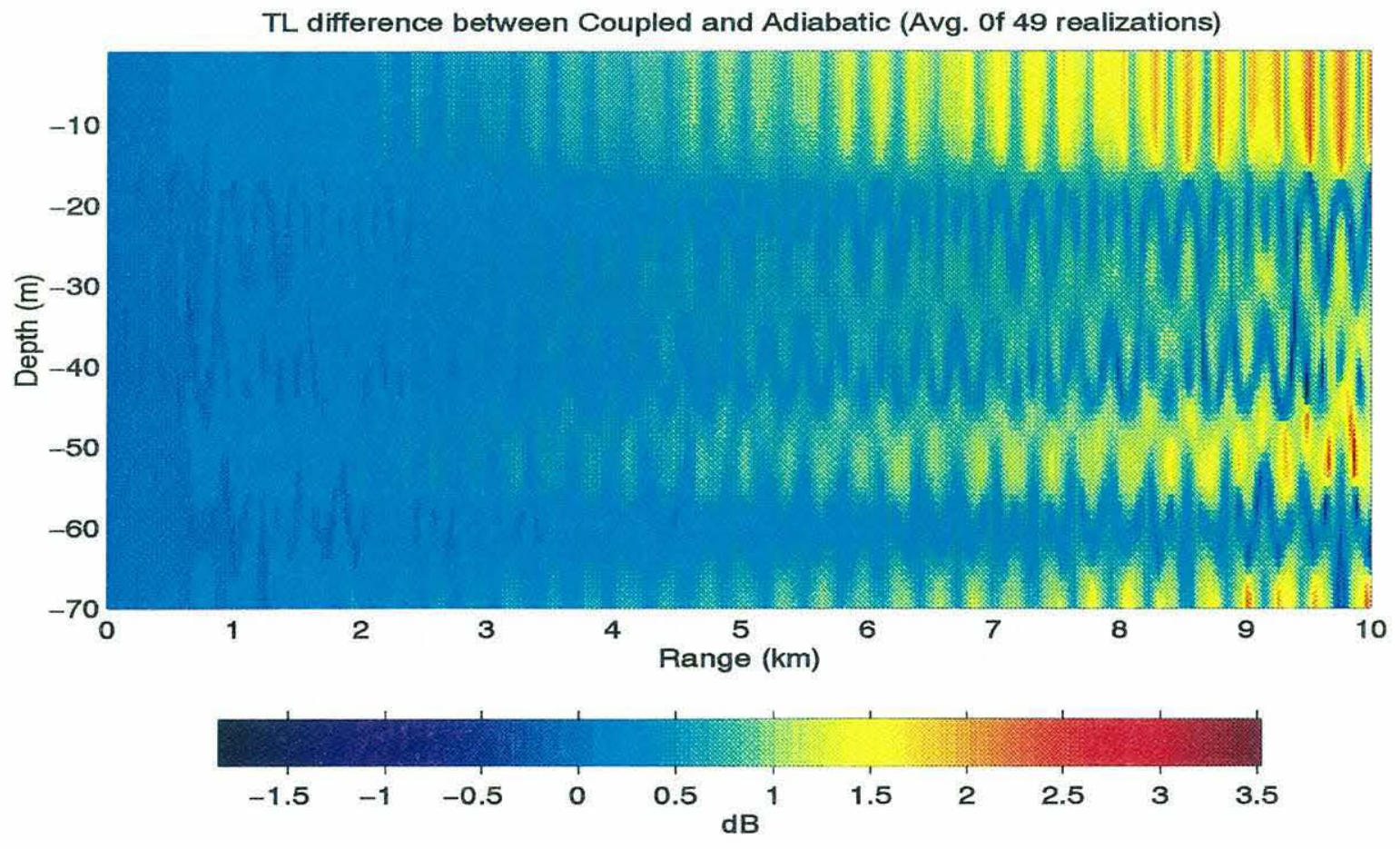

Figure 4.20: Transmission loss difference between adiabatic approximation and coupled mode calculations (average of 49 realizations) for frequency of $100 \mathrm{~Hz}$ and IW amplitude of $30 \mathrm{~m}$. 
TL diference between Adiabatic and Coupled (Avg. of 49 realizations)
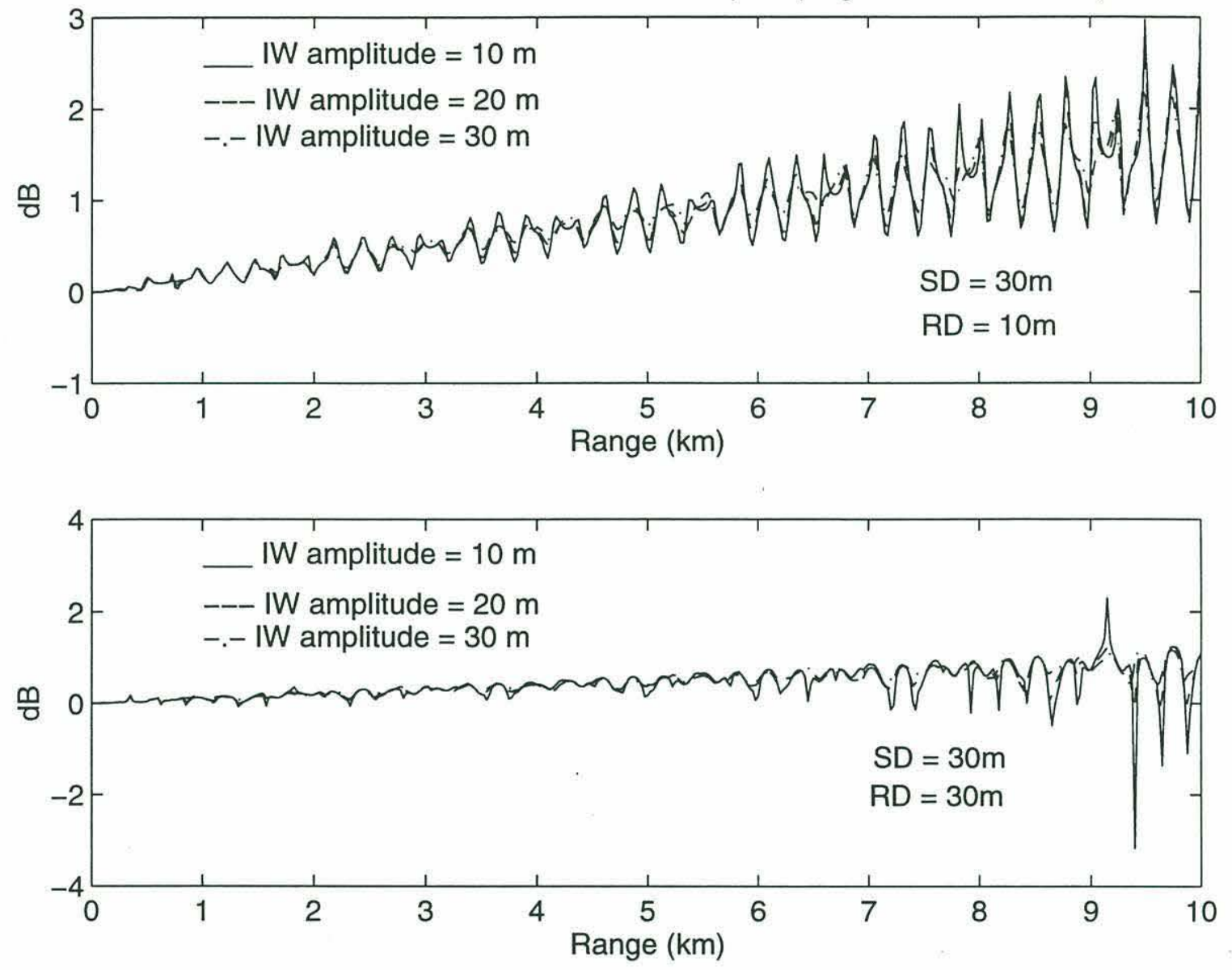

Figure 4.21: TL difference between adiabatic and coupled mode calculations (average of 49 realizations) for IW amplitudes of $10 \mathrm{~m}, 20 \mathrm{~m}$, and $30 \mathrm{~m}$ at receiver depth of $10 \mathrm{~m}$ and $30 \mathrm{~m}$ 


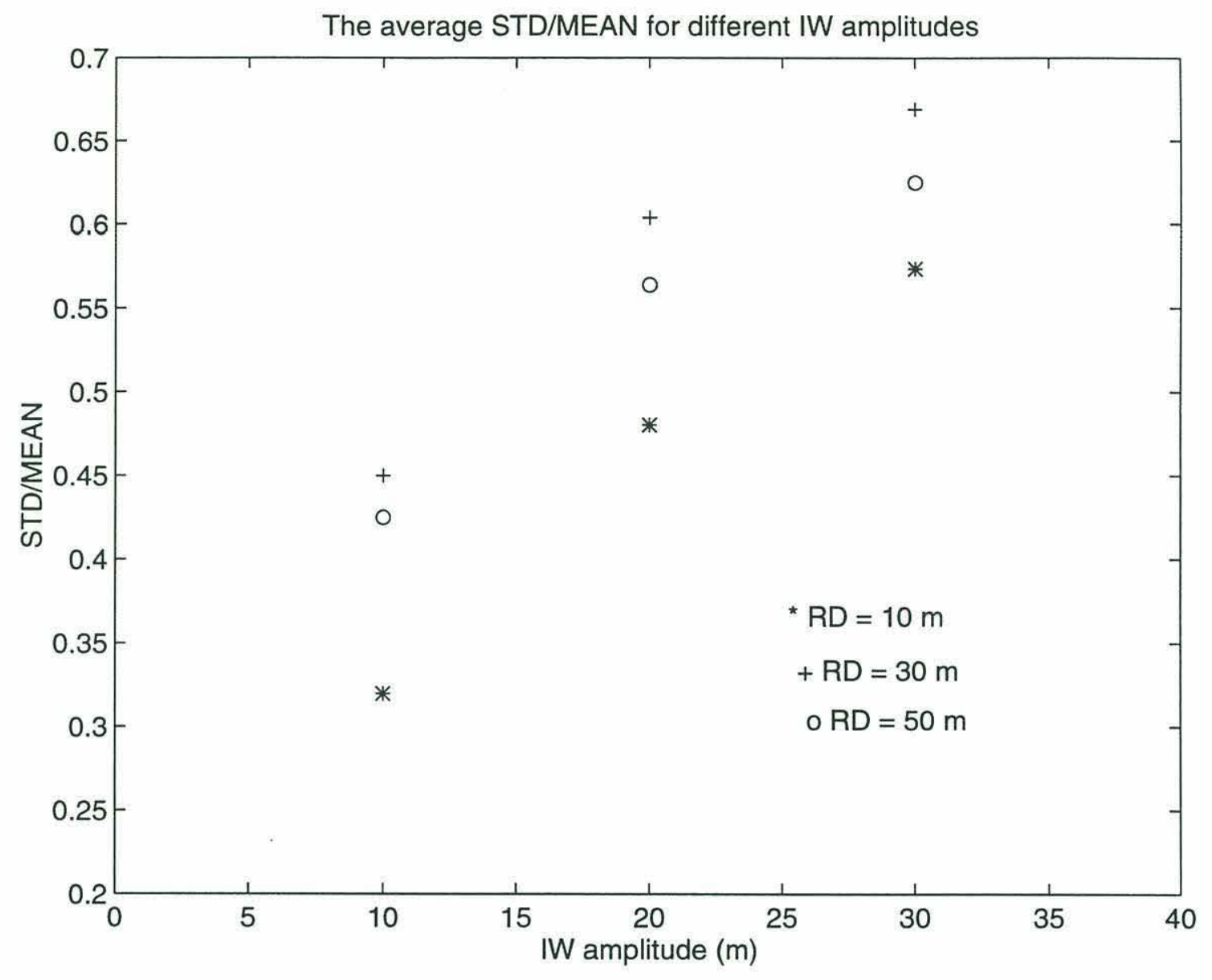

Figure 4.22: The average ratio of standard deviation to mean value of coherent intensity for different IW amplitudes. Frequency $=100 \mathrm{~Hz}$. 


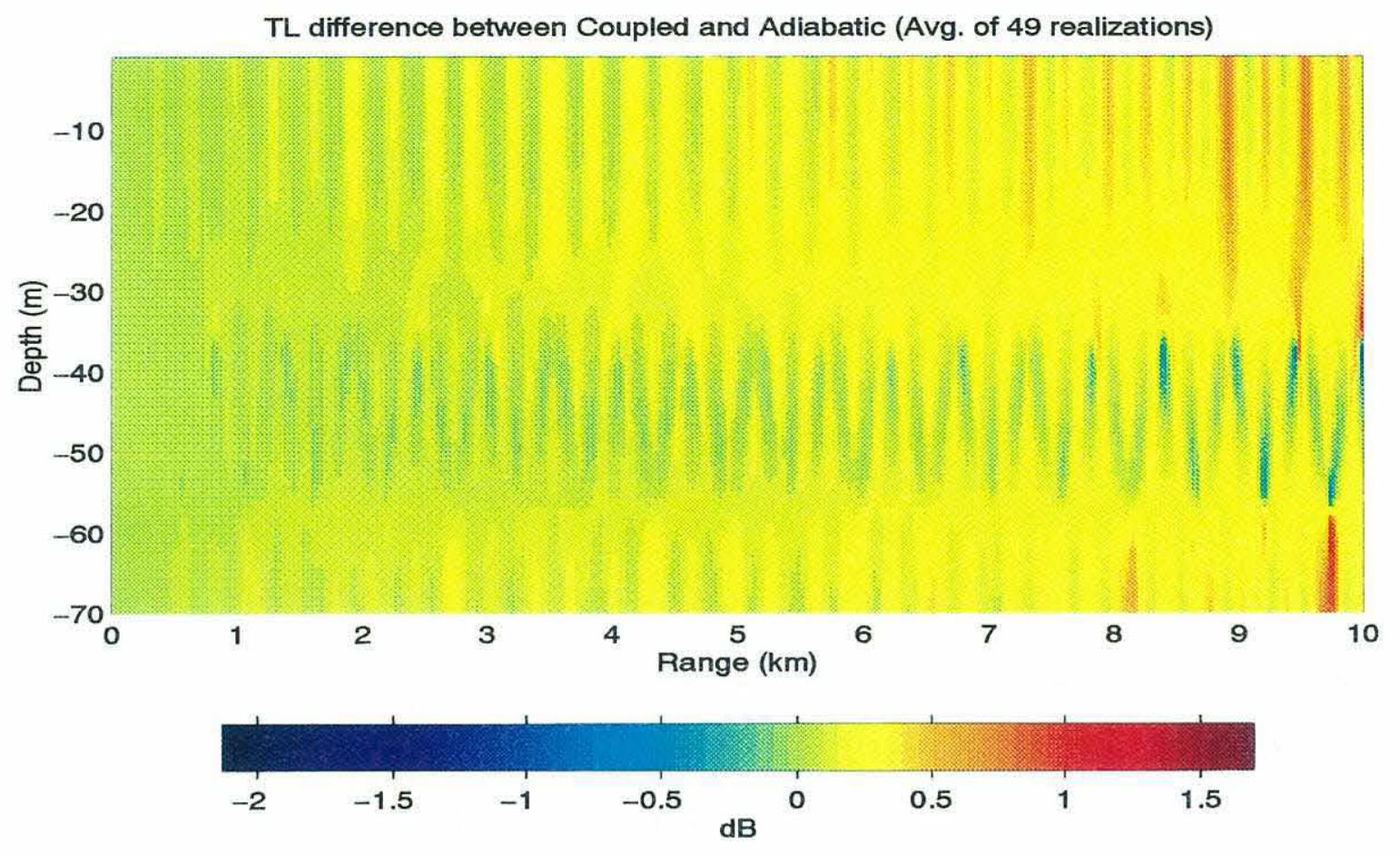

Figure 4.23: TL difference between adiabatic and coupled mode calculations (average of 49 realizations) for frequency of $50 \mathrm{~Hz}$.

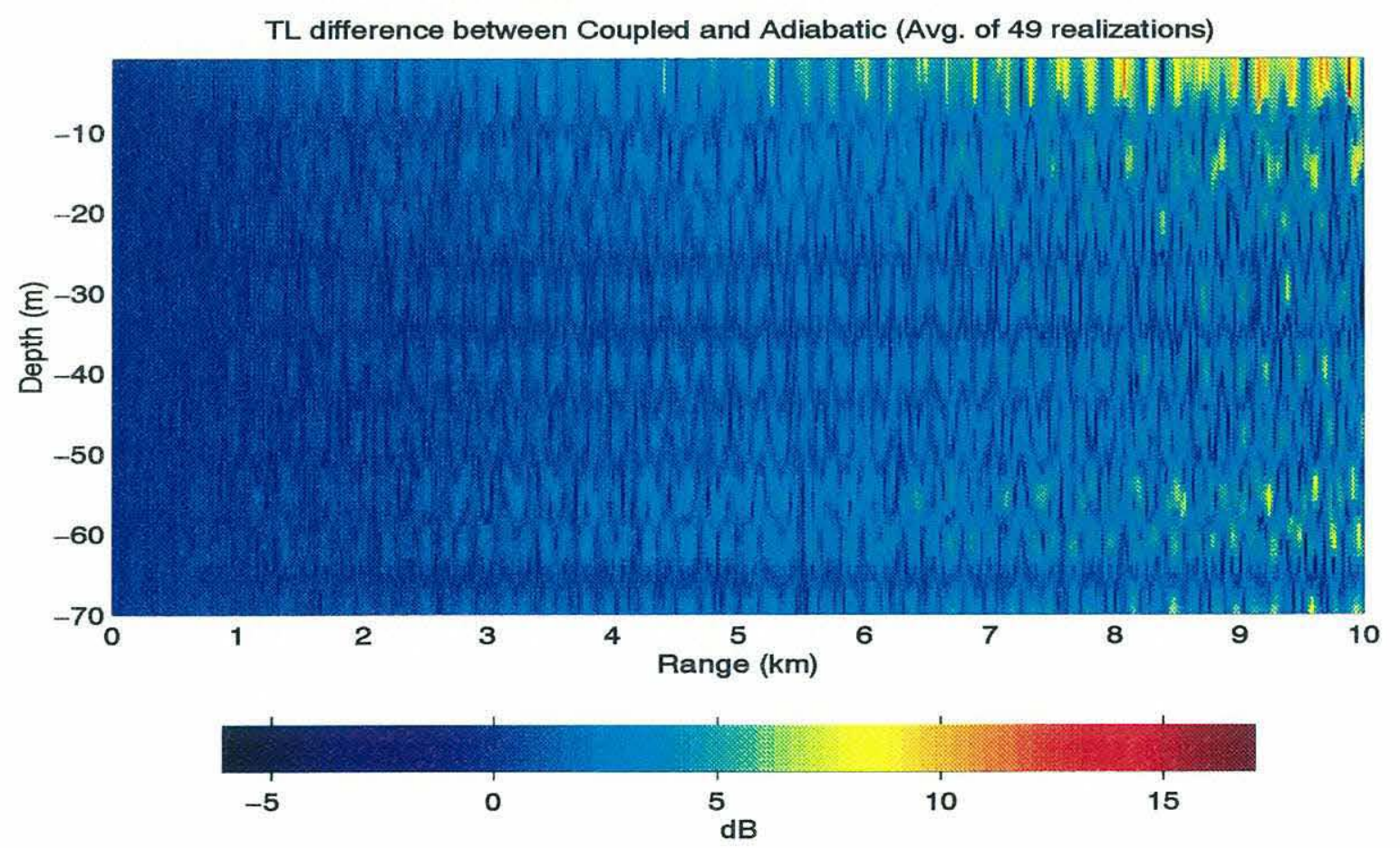

Figure 4.24: Transmission loss difference between adiabatic and coupled mode calculations (average of 49 realizations) for frequency of $200 \mathrm{~Hz}$. 
TL difference between Adiabatic and Coupled (Avg. of 49 realizations)
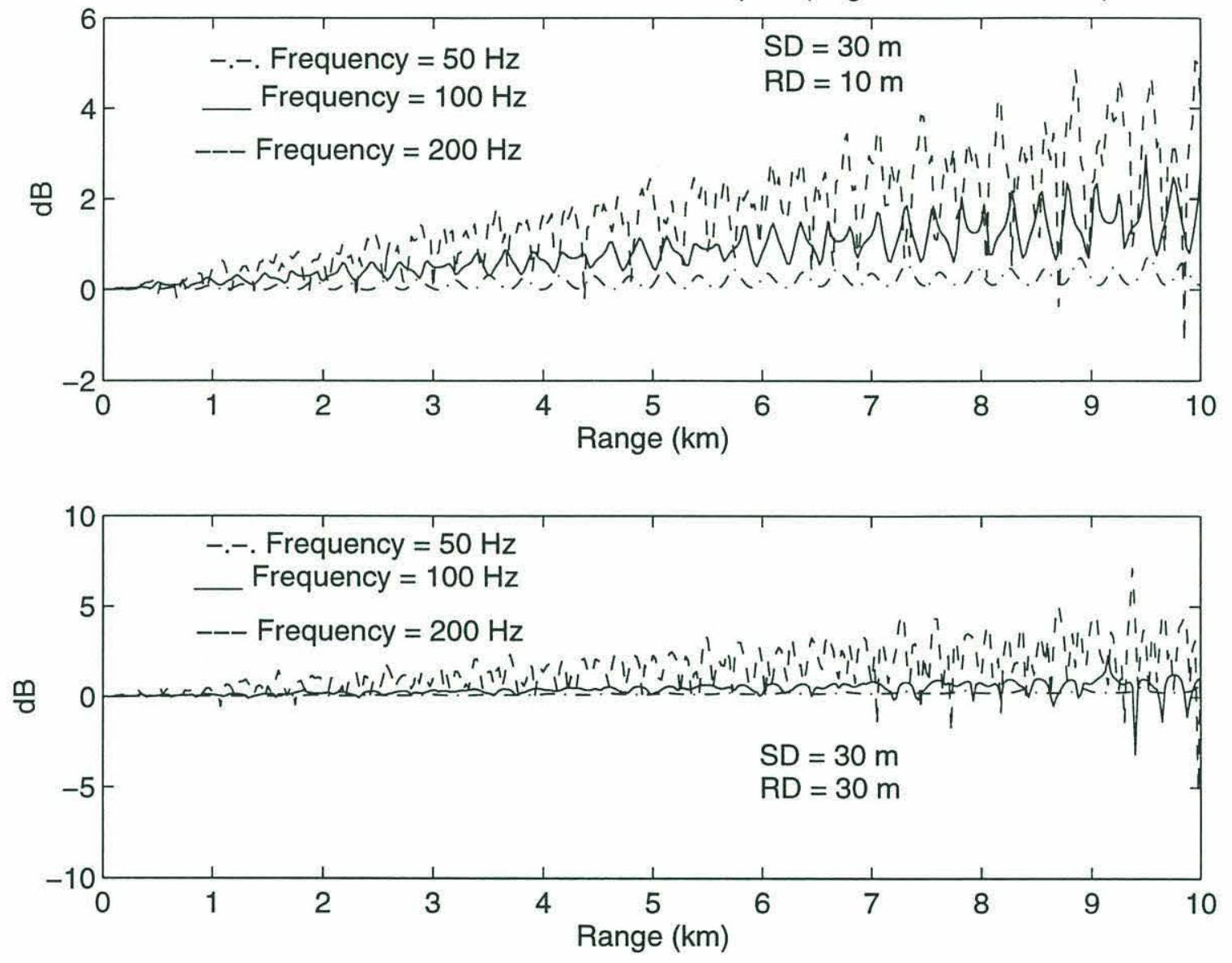

Figure 4.25: TL difference between adiabatic and coupled mode calculations (average of 49 realizations) for different frequencies at receiver depth of $10 \mathrm{~m}$ and $30 \mathrm{~m}$. 


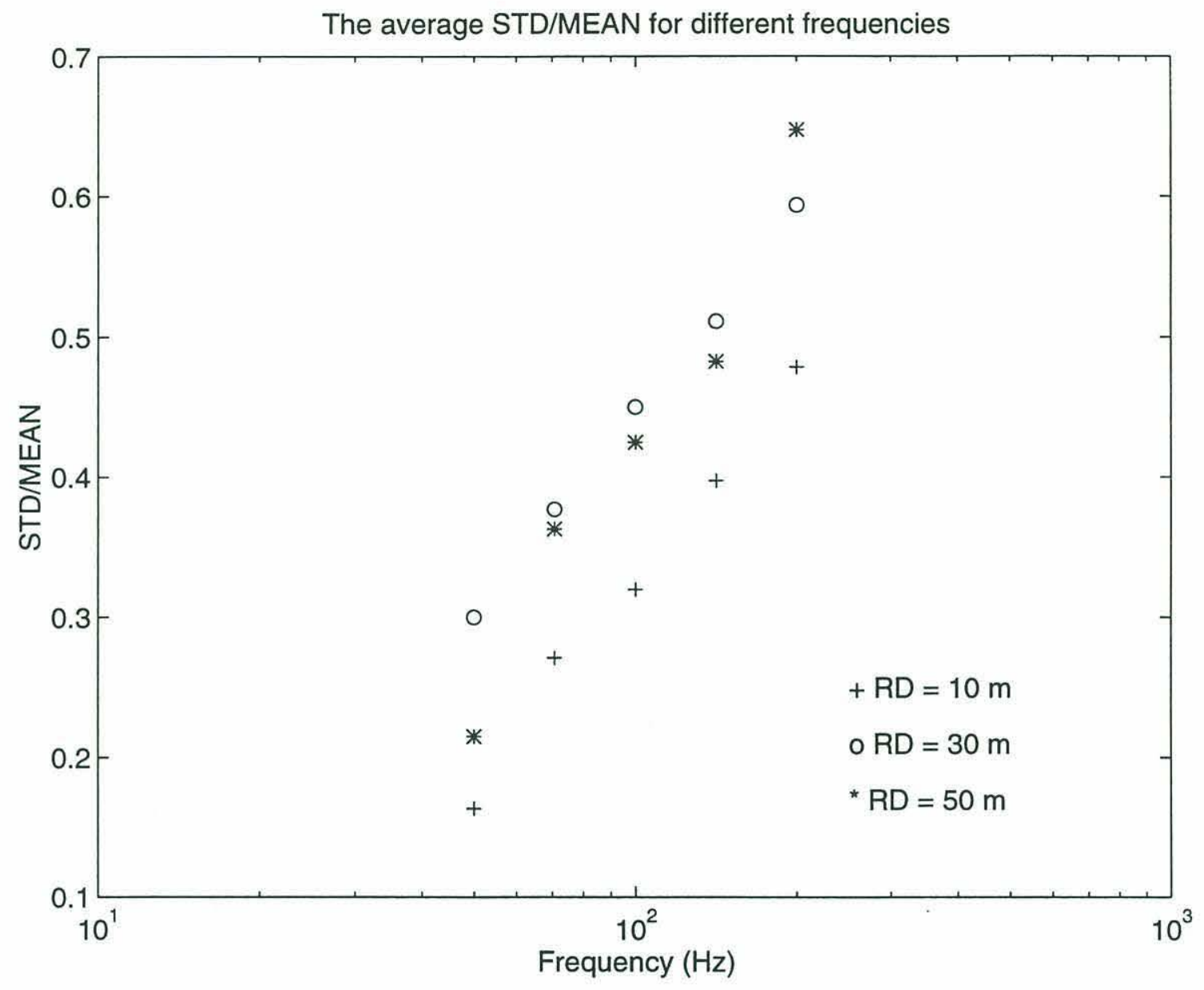

Figure 4.26: The average ratio of standard deviation to mean value of coherent intensity for different frequencies at different receiver depths. IW amplitude and source depth are the same. 


\subsubsection{Incoherent intensity calculation}

The incoherent intensity calculation serves two purposes: one is to inter-compare between coherent and incoherent intensity results; the other is to test the theory of incoherent adiabatic intensity fluctuations derived in Section 3.3. To calculate the incoherent intensity, we modified some of the Kraken programs. For each realization, we repeat the same calculation process as for the coherent intensity. And after 49 realizations, we calculated the mean and variance of the incoherent intensity. Figs. 4.27 and 4.28 show both the coherent and incoherent results. We can notice that in the mid-range, where the variance of coherent intensity is the biggest, the mean of the coherent intensity approaches the incoherent one. This phenomena means that the detailed coherent interference structure may not be very useful in the situation of large IW induced sound speed fluctuations.

Fig. 4.29 shows the 2-D plot of the ratio of the standard deviation to the mean value for the incoherent intensity. Vertically over depth, we can notice the basic mode structure (see Fig. 4.7). However, comparing it with the coherent results shown in Figs. 4.13 and 4.14 , we find that the horizontal structure of the incoherent intensity variance is different from that of the coherent intensity variance in that the incoherent intensity variance doesn't have the horizontal structure in which the intensity variance increases with range first and then decreases until the end of one internal wave cycle. The reason for the difference is that most of the variance of coherent intensity is caused by the mode phase fluctuation which causes the fluctuation of the horizontal mode interference structure that accounts for most of the coherent intensity variance. For the incoherent intensity, according to Eqs.(3.81)-(3.84), the horizontal structure of the variance is determined by the horizontal autocorrelation function of the IW vertical displacement. 
Fig. 4.30 shows the theoretical results for incoherent intensity variance. Comparing it with the Kraken results in Fig 4.29, we find that they agree very well. This tests our theory of the incoherent intensity. Furthermore, the theory predicts that variance of incoherent intensity increases linearly with IW amplitude. We then repeat the incoherent intensity calculations for different IW amplitudes and from the results shown in Fig. 4.31, we also see the linear dependence between the variance and the IW amplitude. This is another test which our theory has passed. 


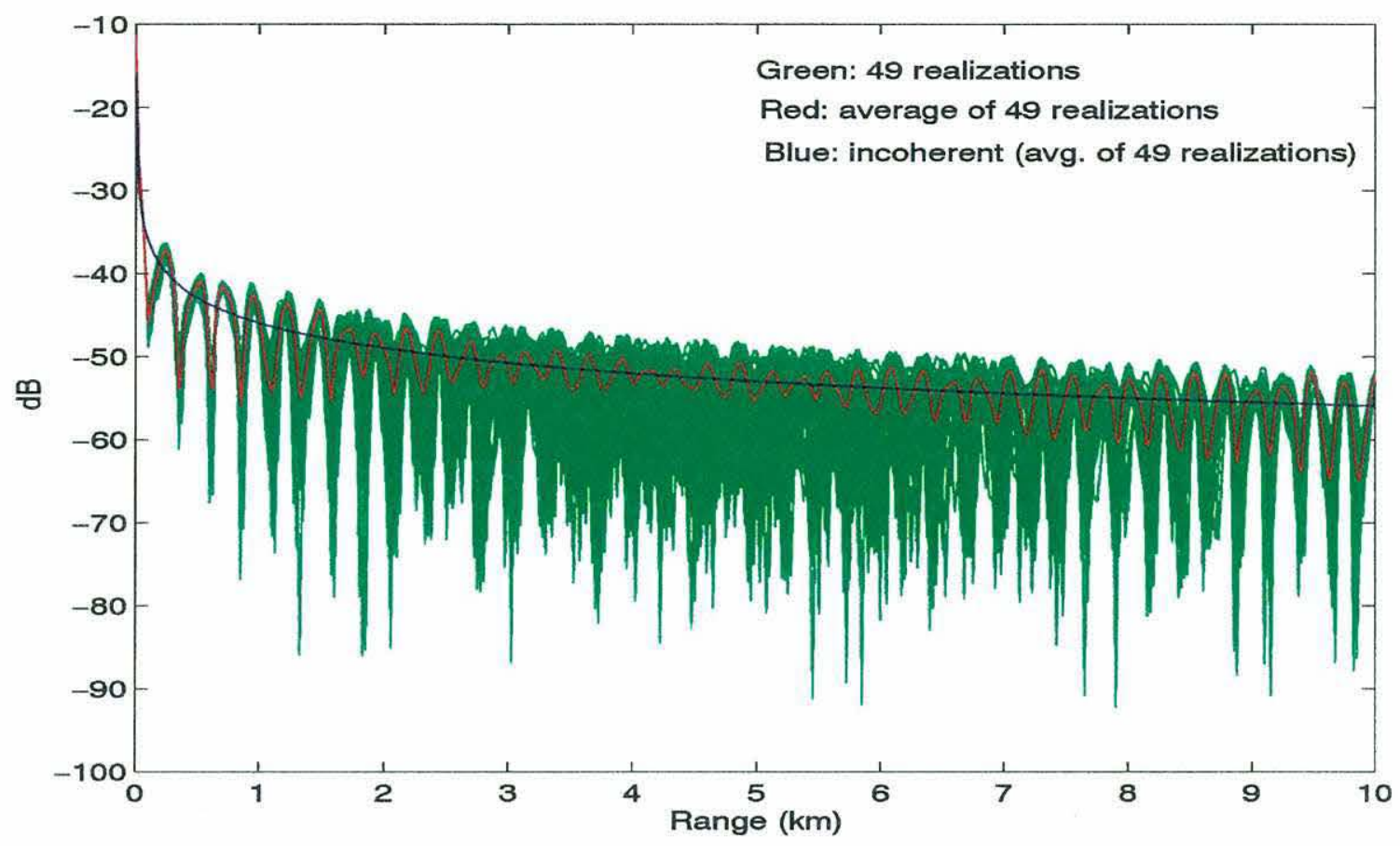

Figure 4.27: Coherent and incoherent transmission loss curves at receiver depth of $30 \mathrm{~m}$ for IW amplitude of $20 \mathrm{~m}$, frequency of $100 \mathrm{~Hz}$ and source depth of $30 \mathrm{~m}$

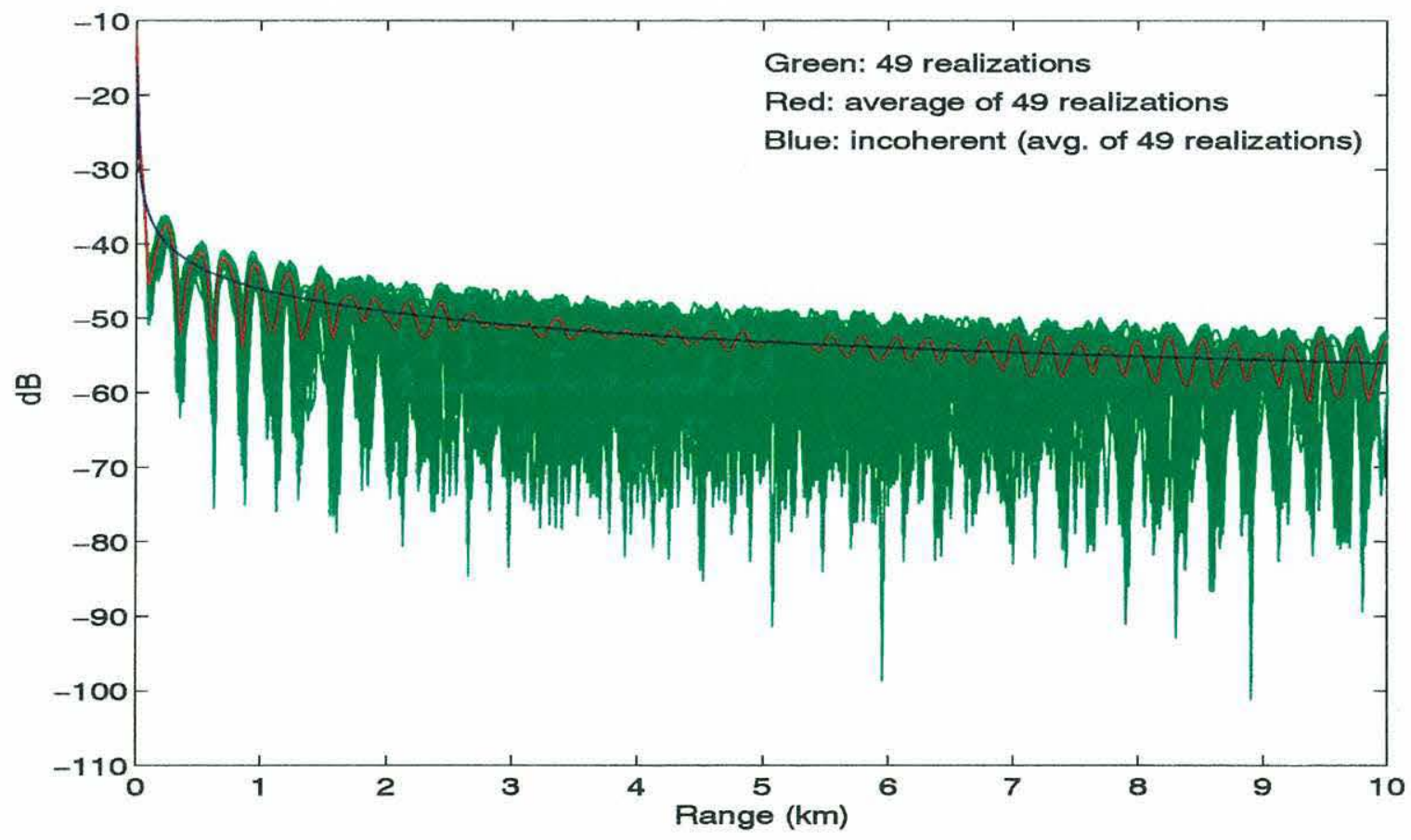

Figure 4.28: Coherent and incoherent transmission loss curves at receiver depth of $30 \mathrm{~m}$ for IW amplitudes of $30 \mathrm{~m}$, frequency of $100 \mathrm{~Hz}$ and source depth of $30 \mathrm{~m}$ 


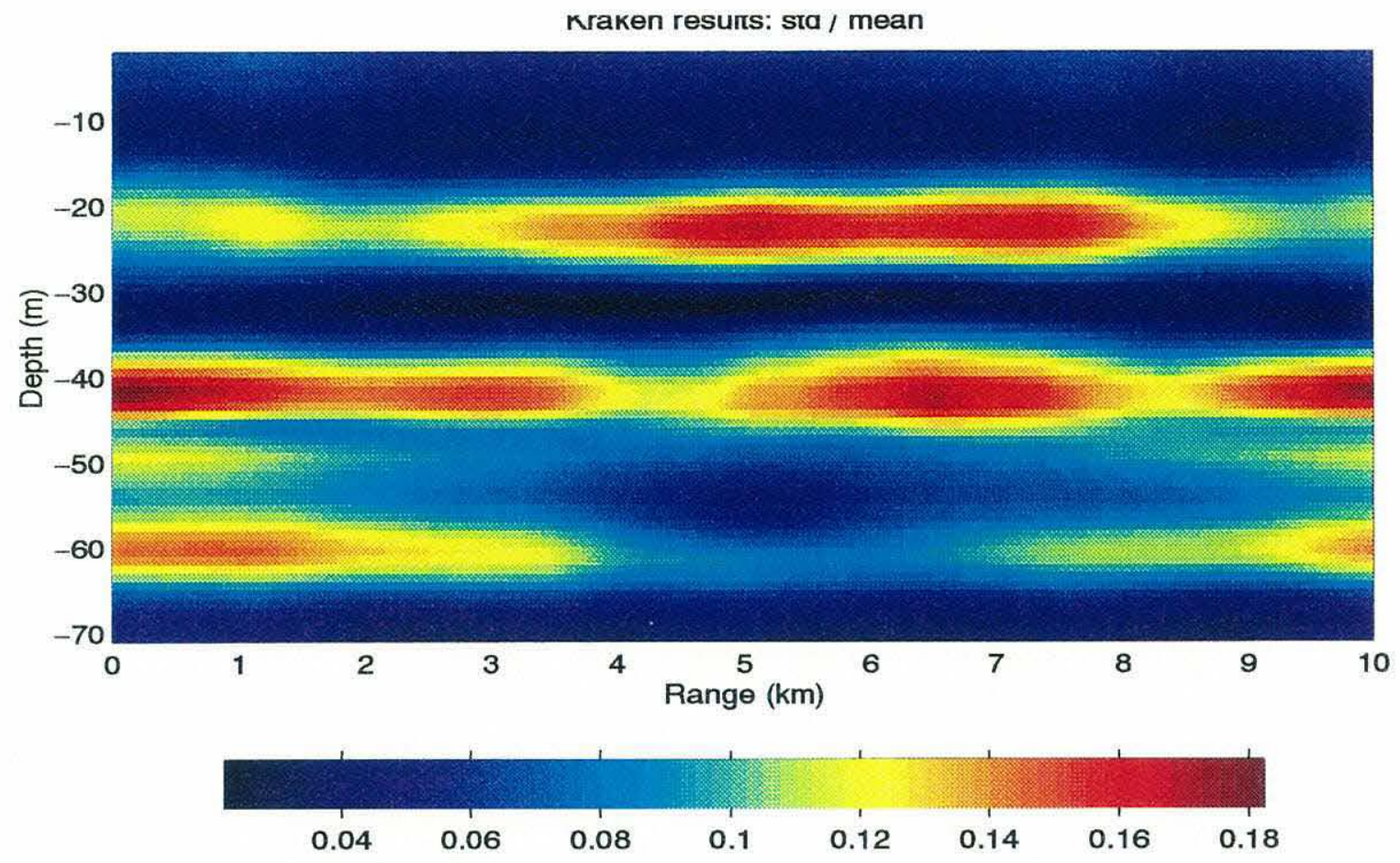

Figure 4.29: The ratio (average of 49 realizations) of standard deviation to mean value for incoherent intensity (Kraken results). Frequency $=100 \mathrm{~Hz}$, IW amplitude $=20 \mathrm{~m}$, and source depth $=30 \mathrm{~m}$.

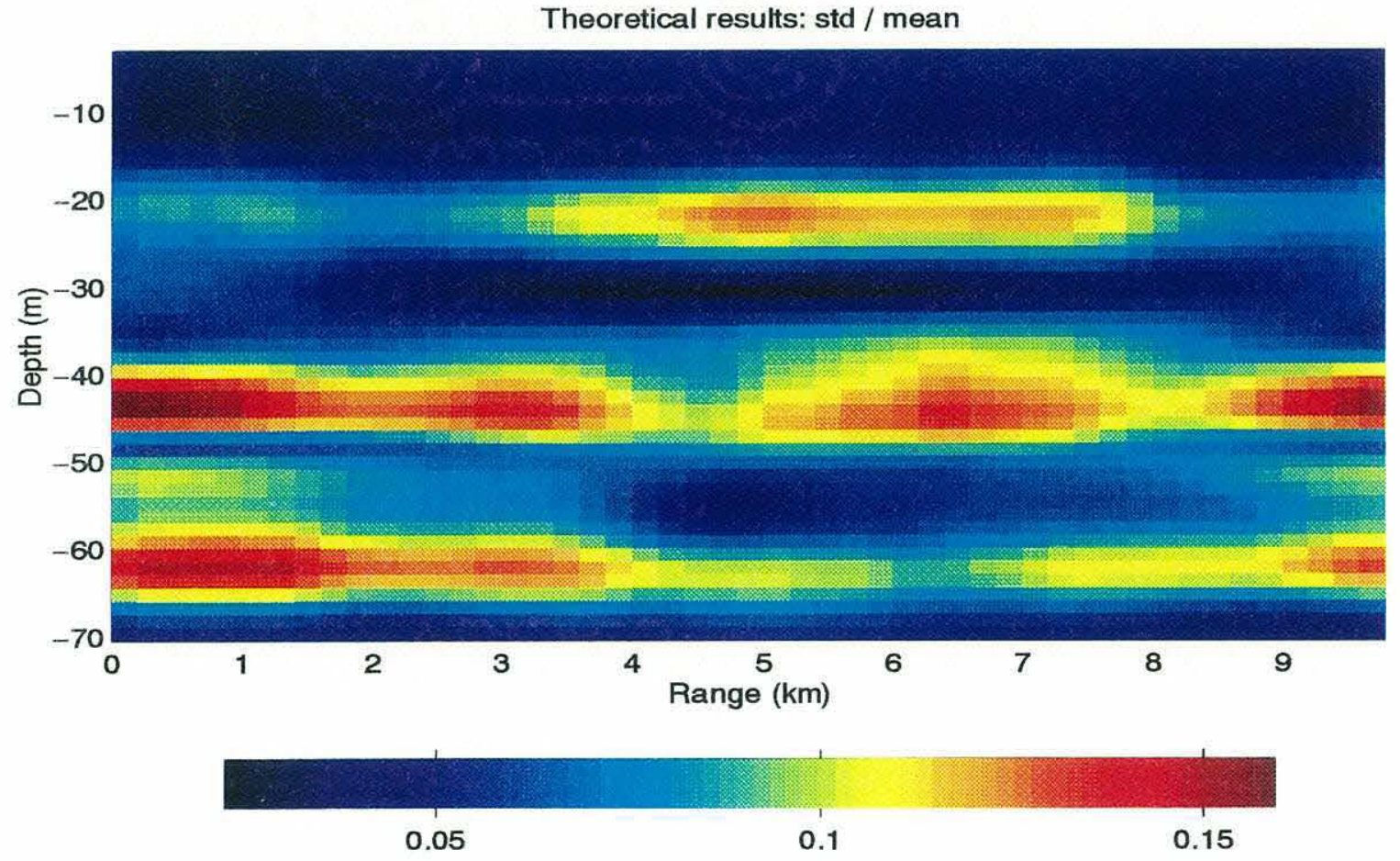

Figure 4.30: The ratio of standard deviation to mean value (average of 49 realizations) for incoherent intensity (theoretical results). Frequency $=100 \mathrm{~Hz}$, IW amplitude $=20 \mathrm{~m}$, and source depth $=30 \mathrm{~m}$ 


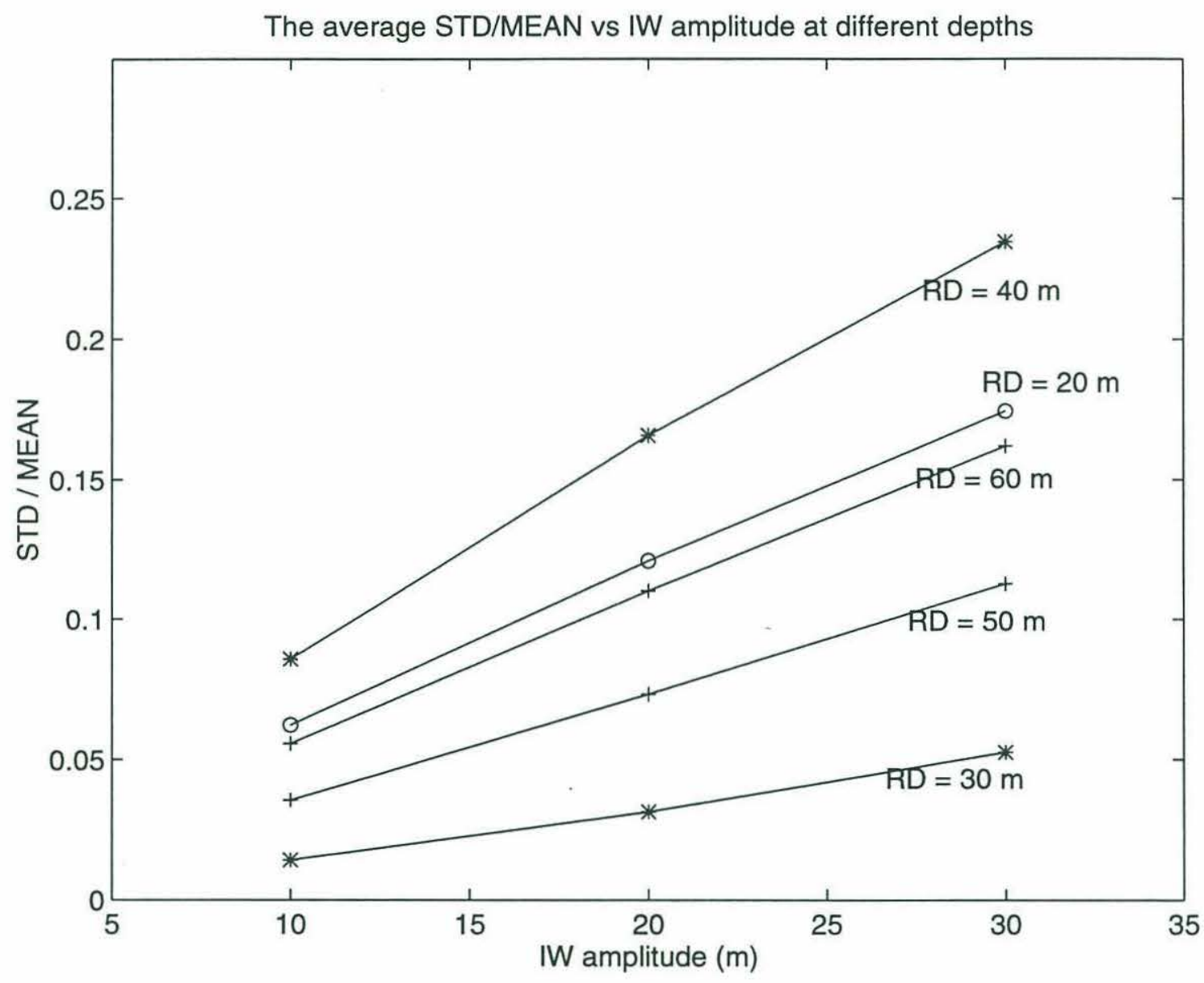

Figure 4.31: The average ratio of standard deviation to mean value for incoherent intensity (Kraken results) vs. IW amplitude at different depths. 


\section{Chapter 5}

\section{Conclusions and future work}

\subsection{Conclusions:}

This thesis investigated the amplitude fluctuation effects in acoustic scattering due to shallow water internal waves. It included two parts: theoretical and numerical. Theoretically, for the adiabatic approximation, we derived expressions for incoherent intensity fluctuation and analyzed the range dependence of the coherent and incoherent intensity fluctuations and for the coupled mode method, we analyzed acoustic mode coupling due to internal waves. The theoretical conclusions we make from this thesis are as follows:

(1) The incoherent intensity fluctuations are mainly caused by the acoustic mode shape perturbations due to IW induced sound speed fluctuations. The range dependence of the incoherent intensity variance is determined by the horizontal autocorrelation function of the IW vertical displacement.

(2) The coherent intensity fluctuations are mainly consequences of the modal horizontal wavenumber perturbations due to IW caused sound speed fluctuations. The range dependence of the coherent intensity variance is determined by the range dependence of the variance of modal phase fluctuations. The variance of modal phase fluctuations, starting from zero at source, is periodic over range with a period of the dominant IW wavelength.

(3) The dominant acoustic mode coupling due to IW's is caused by resonant interaction between sound waves and internal waves. Resonance, i.e. maximum mode coupling, occurs when acoustic mode cycle distance is equal to IW wavelength. For linear internal 
waves, the mode coupling effect increases with range and is proportional to the IW spectrum magnitude at the wavenumber corresponding to the acoustic mode cycle distance.

Numerically, we use the Garrett-Munk IW spectrum model to simulate IW field and use the Kraken programs to evaluate acoustic transmission in the simulated IW field. The conclusions drawn from the numerical results are as follows.

(1) The transmission loss difference between the adiabatic approximation and the coupled mode methods increases with range. This agrees with one of the theoretical conclusion which states that mode coupling effect accumulates over range.

(2) The transmission loss difference between the adiabatic approximation and the coupled mode methods increases with acoustic frequency but barely changes with IW amplitude. The explanation is that the TL difference depends on the ratio of IW energy contained in the dominant wavelengths to IW energy contained in the wavelengths corresponding to the dominant acoustic mode cycle distances. And the ratio doesn't change with pure IW amplitude changes (since the spectrum shape doesn't change) while it changes with acoustic frequency. The frequency dependence has to do with modal cycle distance changes and mode amplitude changes with frequency.

(3) The variance of amplitude fluctuations increases with frequency and IW amplitude for both incoherent and coherent intensities. This agrees with the theoretical conclusions very well. Also, the horizontal structures of the intensity variances from numerical calculations provide excellent test of our theoretical work. 


\subsection{Recommendations for future work}

Theoretically, this thesis investigates the incoherent intensity fluctuations due to IW's using adiabatic approximation. Detailed quantitative evaluation of coherent intensity fluctuation in theory, if possible, could be the next step to investigate the amplitude fluctuation effects in acoustic scattering due to IW's. Also, theoretical treatment of transmission loss difference between adiabatic approximation and coupled mode method is a very important topic. Numerically, we can include more complex IW models, solitons, and more realistic bottom models. We could also add volume attenuation and surface and bottom reflection attenuation to the numerical model. PE (parabolic equation) method could also be used to evaluate the acoustic field.

This thesis focused on single frequency acoustic transmission in the IW field. Theoretical and numerical analysis of broadband case would be a very interesting topic. So are higher frequency and longer range cases. Also, spatial statistics (coherence, correlation) of the acoustic transmission in the IW field could be very important for sonar performance and may contain information for stochastic tomography.

Experimental exploration of amplitude fluctuation effect due to IW's is especially important. If we can get experimental data on acoustic transmission as well as oceanographic, geophysical and acoustic properties in real IW field, we can use the experimental property data as input to our numerical programs to evaluate acoustic transmission. We can also theoretically calculate statistics of acoustic scattering due to IW's using these data. Inter-comparisons between theoretical, numerical and experimental results could enable us to better understand the acoustic scattering due to IW's. 


\section{Appendix A}

\section{Calculations of eigenvalue and eigenfunction perturba- tions}

We start from the modal equation

$$
H \Psi_{m}=E_{m} \Psi_{m}
$$

to evaluate the eigenvalue and eigenfunction perturbations due to the perturbation

$$
H=H^{(0)}+H^{\prime} .
$$

We can write the perturbed eigenvalue $E$, and eigenfunction $\Psi_{m}$ as

$$
\begin{gathered}
E_{m}=E_{m}^{(0)}+\lambda E_{m}^{(1)}+\ldots \\
\Psi_{m}=\Psi_{m}^{(0)}+\lambda \Psi_{m}^{(0)}+\ldots
\end{gathered}
$$

Substituting (A.2)-(A.4) into (A.1), matching the orders in the perturbation expansion equation, we obtain

$$
H^{(0)} \Psi_{m}^{(0)}=E_{m}^{(0)} \Psi_{m}^{(0)}
$$

and

$$
H^{(0)} \Psi_{m}^{(1)}+H^{\prime} \Psi_{m}^{(0)}=E_{m}^{(0)} \Psi_{m}^{(1)}+E_{m}^{(1)} \Psi_{m}^{(0)}
$$

The function $\Psi_{m}^{(1)}$ can be expanded in terms of the zeroth order problem eigenstates $\Psi_{m}^{(0)}$ (which are a complete set of states), i.e. 


$$
\Psi_{m}^{(1)}=\sum_{n} a_{m n} \Psi_{n}^{(0)}
$$

Substituting (A.7) into (A.6), we obtain

$$
\sum_{n} a_{m n} H^{(0)} \Psi_{n}^{(0)}+H^{\prime} \Psi_{m}^{(0)}=E_{m}^{(0)} \sum_{n} a_{m n} \Psi_{n}^{(0)}+E_{m}^{(1)} \Psi_{m}^{(0)}
$$

Substituting (A.5) into (A.8) gives us

$$
\sum_{n} a_{m n} E_{n}^{(0)} \Psi_{n}^{(0)}+H^{\prime} \Psi_{m}^{(0)}=E_{m}^{(0)} \sum_{n} a_{m n} \Psi_{n}^{(0)}+E_{m}^{(1)} \Psi_{m}^{(0)}
$$

Applying the operator $\int\left({ }^{*}\right) \Psi_{k}^{(0)} d z$ to (A.9) and utilizing the orthogonality

$$
\int \Psi_{m} \Psi_{k} d z=\delta_{m k}
$$

where $\delta_{m k}$ is the Dirac symbol which is equal to 1 when $m=n$ and zero otherwise, we get

$$
E_{k}^{(0)} a_{m k}+\int \Psi_{k}^{(0)} H^{\prime} \Psi_{m}^{(0)} d z=E_{m}^{(0)} a_{m k}+E_{m}^{(1)} \delta_{m k}
$$

When $m=k$, we get the eigenvalue perturbation

$$
E_{m}^{(1)}=\int \Psi_{m}^{(0)} H^{\prime} \Psi_{m}^{(0)} d z
$$

and when $m \neq k$, we get the eigenfunction perturbation coefficients

$$
a_{m k}=\frac{1}{E_{m}^{(0)}-E_{k}^{(0)}} \int \Psi_{m}^{(0)} H^{\prime} \Psi_{k}^{(0)} d z
$$


Now, specifically, we can evaluate the eigenvalue and eigenfunction perturbations for the modal equation (3.3) (we assume the density $\rho(z)$ as a constant here). Comparing Eq.(3.3) with Eq.(A.1), we find that

$$
\begin{gathered}
H^{(0)}=\frac{d^{2}}{d z^{2}}+k_{0}^{2} \\
H^{\prime}=\delta\left(k^{2}\right)=2 k_{0} \delta k \\
E_{m}=k_{r m}^{2} .
\end{gathered}
$$

Substituting Eqs.(A.14) - (A.16) into Eq.(A.12) and (A.13), we find that the eigenvalue perturbation for Eq.(3.3) is

$$
\delta\left(k_{r m}^{2}\right)=2 k_{r m}^{(0)} \delta k_{r m}=\int 2 k_{0} \delta k\left|\Psi_{m}^{(0)}\right|^{2} d z
$$

i.e.

$$
\delta k_{r m}=\frac{1}{k_{r m}^{(0)}} \int k_{0} \delta k\left|\Psi_{m}^{(0)}\right|^{2} d z
$$

and the eigenfunction perturbation for Eq.(3.3) is

$$
\delta \Psi_{m}=\sum_{k} a_{m k} \Psi_{k}^{(0)}
$$

where

$$
a_{m k}=\frac{1}{k_{r m}^{(0) 2}-k_{r k}^{(0) 2}} \int 2 k_{0} \delta k \Psi_{m}^{(0)} \Psi_{k}^{(0)} d z
$$




\section{References}

[1] S. M. Flatte (ed), Sound Transmission through a Fluctuating Ocean, Cambridge University Press (1979).

[2] C. Clay and H. Medwin, Acoustic Oceanography, Wiley, New York (1977).

[3] L.B. Dozier and F.D. Tappert, "Statistics of normal mode amplitudes in a random ocean. I. Theory”, J. Acoust. Soc. Am. 63(2), 353-365 (1978).

[4] -, "Statistics of normal mode amplitudes in a random ocean. II. Computations", J. Acoust. Soc. Am. 64(2), 533-547 (1978)

[5] J.X.Zhou, X.Z.Zhang, and P.H.Rogers, "Resonant interaction of sound wave with internal solitons in the coastal ocean", J. Acoust. Soc. Am. 90 (6), 3435-33437 (1992).

[6] H.H.Essen, F.Schirmer, and S. Sirkes, "Acoustic remote sensing of internal waves in shallow water", Int. J. Remote Sensing, 4(1), 33-47 (1983).

[7] A.Yu. Shmelerv, A.A.Migulin, and V.G. Petvikov, "Horizontal refraction of low frequency acoustic waves in the Barents Sea stationary acoustic track experiment", J. Acoust. So. Am. 92(2), 1003-1007 (1992).

[8] D. Rubenstein and M.H. Brill, "Acousic variability due to internal waves and surface waves in shallow water", in Ocean Variability and Acoustic Propagation, edited by J. Potter and A. Warn-Varnas (Kluwer Academic, Dordrecht, 1991) pp.215-228.

[9] J.F. Lynch, G. Jin, R. Pawlowicz, D. Ray, C.S. Chiu, and J. Miller, R.H. Bourke, R. Parson, A. Pluedemann, R. Muench, "Acoustic travel time perturbations due to shallow water internal waves and internal tides in the Barents Sea Front: theory and experiment", submitted to J. Acoust. Soc. Am. (1995).

[10] P. Traykovski, "Travel time perturbations due to internal waves: equivalence of modal and ray solutions", submitted to J. Acoust. Soc. Am. (1995).

[11] W.A. Anikouchine and R.W. Sternberg, The World Ocean: an Introduction to Oceanography, Prentice-Hall, Englewood Cliffs, NJ (1981).

[12] A.E. Gill, Atmosphere-Ocean Dynamics, Academic Press, New York (1982).

[13] C. Garrett and W. Munk, "Internal waves in the ocean", Ann. Rev. Fl. Mech. 19, 339369 (1979).

[14] J.R. Apel, H.M. Byrne, J.R. Proni, and R.L. Charnell, "Observations of oceanic internal and surface waves from the Earth Resources Technology Satellite", J. Geophy. Research, 80(6), 865-881 (1995).

[15] L.L Fu and B. Holt, "Internal waves in the Gulf of California: observations from a spaceborne radar", J. Geophysical Research, 89(C2), 2053-2060 (1984).

[16] M.H. Orr, "Remote acoustic sensing of the oceanic fluid and biological processes", Woods Hole Oceanogr. Inst. Tech. Rep. WHOI-80-2 (1980).

[17] C. Garrett and W. Munk, "Space-time scales of internal waves: a progress report", J. Geophysical Research, 80 (3), 291-297 (1975).

[18] L.A. Ostrovsky and Yu. A. Stepanyants, "Do internal solitons exist in the ocean?", Reviews of Geophysics, 27, 3, 293-310 (1989).

[19] L. Brekhovskikh and Yu. Lysanov, Fundamentals of Ocean Acoustics, Springer-Verlag, Berlin (1982). 
[20] Finn B. Jensen, William A. Kuperman, Michael B. Porter, Henrik Schmidt, Computational Ocean Acoustics, American Institute of Physics Press, New York (1994).

[21] A.D. Pierce, "Extension of the method of normal modes to sound propagation in an almost stratified medium", J. Acoust. Soc. Am. 37, 19-27 (165).

[22] S.D. Rajan, J.F. Lynch, and G.V. Frisk, "Perturbative inversion methods for obtaining bottom geoacoustic parameters in shallow water", J. Acoust. Soc. Am. 82, 998-1017 (1987)

[23] S.T. MacDaniel, "Mode coupling due to interaction with the seabed", J. Acoust. Soc. Am., 73(3), 916-923, 1982.

[24] Michael B. Porter, The KRAKEN Normal Mode Program, SACLANT Undersea Research Center, 1992.

[25] Arthur Newhall, Documentation on using Kraken, Woods Hole Oceanographic Institution, 1995. 